\section{Aggregation-induced emission}

\author{
Yuning Hong, ${ }^{a b}$ Jacky W. Y. Lam ${ }^{a b}$ and Ben Zhong Tang*abc \\ Received 26th April 2011 \\ DOI: $10.1039 / \mathrm{c} 1 \mathrm{cs} 15113 \mathrm{~d}$
}

Luminogenic materials with aggregation-induced emission (AIE) attributes have attracted much interest since the debut of the AIE concept in 2001. In this critical review, recent progress in the area of AIE research is summarized. Typical examples of AIE systems are discussed, from which their structure-property relationships are derived. Through mechanistic decipherment of the photophysical processes, structural design strategies for generating new AIE luminogens are developed. Technological, especially optoelectronic and biological, applications of the AIE systems are exemplified to illustrate how the novel AIE effect can be utilized for high-tech innovations (183 references).

\section{Introduction}

Light is extremely important to human beings, as reflected by the scriptural description in Genesis 1 that the first creative act of God was to generate light. Light is not only useful but also mysterious to mankind, which has stimulated much effort from the early stage of civilization to decipher light-emitting processes. Classic studies of organic luminescence have generally been conducted in the solution state. ${ }^{1-5}$ For example, photophysical parameters, such as exciton lifetime, decay rate and luminescence

${ }^{a}$ Department of Chemistry, Nano Science and Technology Program, Bioengineering Graduate Program, Institute of Molecular Functional Materials, The Hong Kong University of Science \& Technology

(HKUST), Clear Water Bay, Kowloon, Hong Kong, China.

E-mail: tangbenz@ust.hk

${ }^{b}$ HKUST Fok Ying Tung Research Institute, Nansha, Guangzhou, China

${ }^{c}$ Department of Polymer Science \& Engineering, Institute of Biomedical Macromolecules, MoE Key Laboratory of

Macromolecular Synthesis and Functionalisation,

Zhejiang University, Hangzhou 310027, China efficiency, have usually been measured or determined in highly dilute solutions, where the luminophoric molecules can be approximated as isolated species without being perturbed to an appreciable extent by chromophoric interactions.

The solution investigations have made great contributions to the fundamental understanding of luminescence processes at molecular level. The conclusions drawn from the dilutesolution data, however, cannot commonly be extended to the concentrated solutions. Indeed, many organic luminophores show very different light-emitting behaviours in dilute and concentrated solutions. For example, luminescence is often weakened or quenched at high concentrations, a phenomenon widely known as "concentration quenching". ${ }^{6}$ A main cause for the quenching process is mechanistically associated with the "formation of aggregates", 6,7 which is probably why the concentration quenching effect has frequently been referred to as "aggregation-caused quenching" (ACQ).

An example of the ACQ effect is shown in Fig. 1. The dilute solution $(10 \mu \mathrm{M})$ of $N, N$-dicyclohexyl-1,7-dibromo-3,4,9,10perylenetetracarboxylic diimide (DDPD) in THF is highly

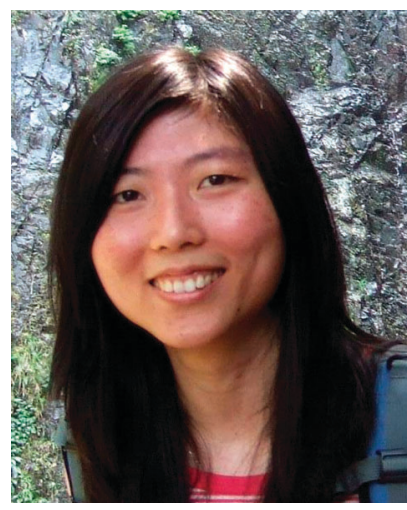

Yuning Hong
Yuning Hong received her BS degree from Sun Yat-sen University in 2006. She conducted her graduate study under the supervision of Prof. Tang and obtained PhD degree from HKUST in 2011. She is now doing her postdoctoral research in the Tang group. Her research is mainly on the development of new AIE molecules and exploration of their biological applications.

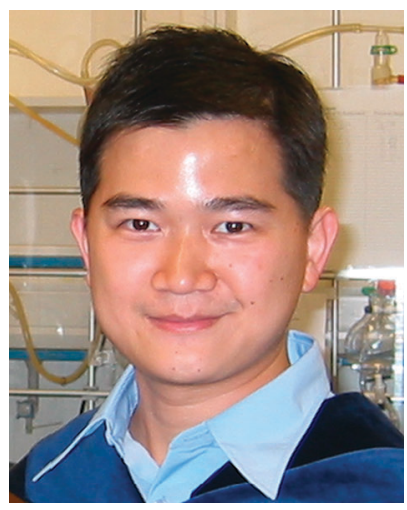

Jacky $W$. Y. Lam received his PhD degree from HKUST in 2003 under the supervision of Prof. Tang. In 2003-2007, he carried out his postdoctoral work on novel polymers with linear and hyperbranched structures and advanced functional properties in the Tang group. He is currently a research assistant professor of chemistry at HKUST. 


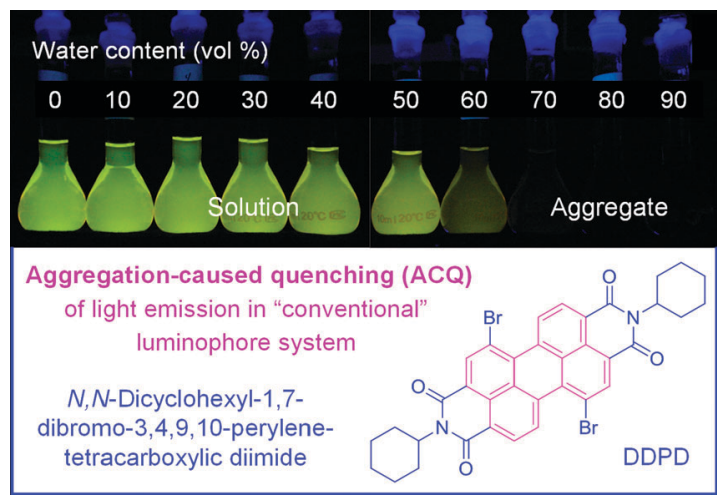

Fig. 1 Fluorescence photographs of solutions/suspensions of DDPD $(10 \mu \mathrm{M})$ in $\mathrm{THF} /$ water mixtures with different water contents.

luminescent. Its emission is weakened when water is added into THF, because the immiscibility of DDPD with water increases the local luminophore concentration and causes DDPD molecules to aggregate. When the water content is increased to $>60 \mathrm{vol} \%$, the solvating power of the THF/water mixture becomes so poor that most of the DDPD molecules become aggregated. As a result of the aggregate formation, the light emission of DDPD is completely quenched. The DDPD molecule contains a disc-like perylene core. In the DDPD aggregates, the perylene rings may experience strong $\pi-\pi$ stacking interactions. This prompts the formation of such detrimental species as excimers, thus leading to the observed ACQ effect (Chart 1A).

As summarized by Birks in his classic photophysics book, the ACQ effect is "common to most aromatic hydrocarbons and their derivatives". ${ }^{6,7}$ A structural reason for the ubiquity of the ACQ effect is because conventional luminophores are typically comprised of planar aromatic rings (e.g., perylene). Since organic luminescence is mainly dictated by electronic conjugation, a popular structural design strategy has been to increase the extent of $\pi$-conjugation by melding more and more aromatic rings together. The resulting bigger discotic

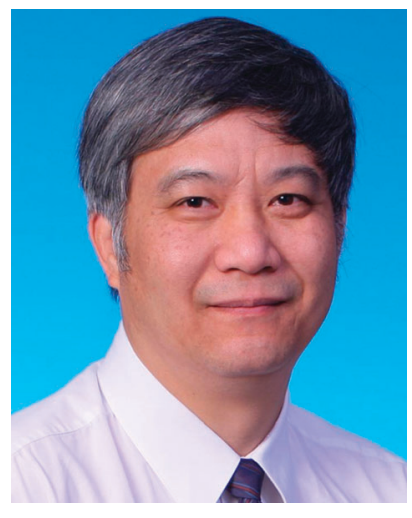

Ben Zhong Tang

elected to the Chinese Academy of Sciences in 2009. He is serving as News Contributor to Noteworthy Chemistry (ACS), Editor-in-Chief of Polymer Chemistry Series (RSC), and Editor of Polymer Bulletin (Springer).

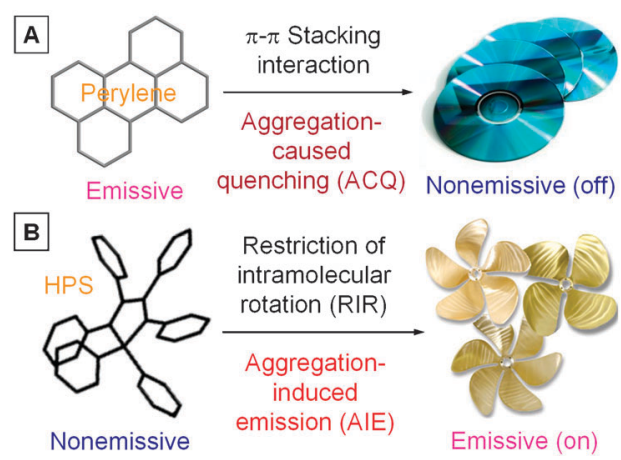

Chart 1 (A) Planar luminophoric molecules such as perylene tend to aggregate as discs pile up, due to the strong $\pi-\pi$ stacking interactions between the aromatic rings, which commonly turns "off" light emission. (B) Non-planar luminogenic molecules such as hexaphenylsilole (HPS) behave oppositely, with their light emissions turned "on" by aggregate formation, due to the restriction of the intramolecular rotation (RIR) of the multiple phenyl rotors against the silole stator in the aggregate state.

plates can indeed luminesce more efficiently in the solutions but concurrently their ACQ effects become severer because the chances for such large luminophores to form excimers or exciplexes are also increased. The ACQ effect has also been observed in inorganic luminophore systems such as quantum dots. ${ }^{89}$ The topic, however, will not be further elaborated, as the focus of this review is on organic systems.

The ACQ effect is generally considered detrimental from the viewpoint of real-world applications. ${ }^{10-14}$ For example, luminophores have been used as sensors to detect biological molecules in physiological buffers and as probes to monitor ionic species in river water. ${ }^{10,12,13}$ Although polar functional groups can be attached to a chromophoric unit to render it hydrophilic, the resultant water-miscible luminophores are still prone to form aggregates in aqueous media due to the unalterable hydrophobicity of their active components of $\pi$-conjugated aromatic rings. The ACQ effect is also a thorny obstacle to the fabrication of efficient organic light-emitting diodes (OLEDs), ${ }^{11}$ where the luminophores are used as thin solid films. In the solid state, the luminophore concentration reaches its maximum owing to the absence of solvent and the ACQ effect hence becomes most severe.

Since the ACQ effect is harmful to practical applications, many research groups have made numerous efforts to tackle the problem. Common manoeuvres include using chemical reactions, physical methods and/or engineering processes to obstruct the formation of luminophoric aggregates. ${ }^{15-19}$ For example, bulky cyclic units are appended to and transparent polymers are blended with chromophores, in order to hinder the luminophores from approaching each other. The uses of the electronically non-conjugated entities, however, can result in shortening of the conjugation length and a drop in the OLED efficiency, owing to the induced twist in the luminophore conformation and barricade to the charge transport. These approaches have thus often ended up with such an embarrassing net effect: an old issue is partly solved but many new problems are created.

The crux of the matter is that these attempts are basically running counter to a natural process, because luminophoric 
molecules have an intrinsic tendency to form aggregates in concentrated solutions or the solid state. Our way of thinking in tackling the problem therefore needs to be fundamentally changed, as it is obviously not so smart to "sail against the current". In 2001, we discovered an uncommon luminogen system, in which aggregation worked constructively, rather than destructively as in the conventional systems. ${ }^{20,21} \mathrm{We}$ found that a series of silole derivatives were non-emissive in dilute solutions but became highly luminescent when their molecules were aggregated in concentrated solutions or cast into solid films. Since the light emission was induced by aggregate formation, we termed the process "aggregationinduced emission" (AIE). ${ }^{20}$

The AIE effect is precisely opposite to the notorious ACQ effect, which makes it possible for technologists to actively utilize the aggregation process, instead of passively working against it. As stated above, photophysical processes in dilute solutions have been extensively investigated $;^{1-5}$ in contrast, luminescence behaviours in aggregate state have been much less studied. The AIE process offers a platform for scientists to look into light emissions from luminogenic aggregates, from which information on structure-property relationships and insights into working mechanisms may be gained. ${ }^{22-28}$ Such information will be instructive to the structural design for the development of efficient luminogens. Since AIE is a photophysical effect about the light emission in the practically useful solid state, the AIE studies may also lead to hitherto impossible technological innovations.

Attracted by the fascinating vistas, many groups have worked on the design and synthesis of new AIE luminogens, preparation and modulation of their aggregate morphologies, and investigation and manipulation of their luminescence behaviours. As a result of the enthusiastic research efforts, a variety of novel AIE systems have been developed, a wealth of mechanistic information has been collected, and a number of practical applications have been explored. In 2009, we published a Feature Article in Chem. Commun. to briefly review our own work in the field of AIE research. ${ }^{29}$ Because of the space limitation, we were unable to cover the excellent studies conducted by other research groups. The rapid advance in the AIE study prompts us to prepare a more comprehensive review article to summarize what has been done and what has been learned in the past decade, with the aim of stimulating new development in this exciting area of research.

The focus of this critical review is thus on the studies undertaken by other research laboratories, although some of our recent results will also be included for discussions. We will first present some typical examples of the AIE systems developed by various research groups, including organic, organometallic and polymeric luminogens, as well as those heretical systems containing no chromophores in the traditional sense. We will then discuss the working mechanisms and operating principles of the AIE processes, in an effort to extract information on their structure-property relationships and derive structural design strategies for creating new AIE systems. Finally, we will recap the research efforts directed towards the exploration of their technological, especially optoelectronic and biological, applications through judicious utilization of the AIE effect.

\section{AIE systems}

Hexaphenylsilole (HPS) is among the first silole derivatives, from which the AIE phenomenon was unearthed. HPS can thus be considered an archetypal AIE luminogen. Structural scrutinization reveals that unlike conventional luminophores such as the disc-like planar perylene, HPS is a propellershaped non-planar molecule (Chart 1). We believe that it is this difference in the molecular structure that has caused the difference in the emission behaviour. In a dilute solution, six phenyl rotors in an HPS molecule undergo dynamic intramolecular rotations against its silacyclopentadiene or silole stator, which non-radiatively annihilates its excited state and renders its molecule non-luminescent. In the aggregates, the HPS molecules cannot pack through a $\pi-\pi$ stacking process due to its propeller shape, while the intramolecular rotations of its aryl rotors are greatly restricted owing to the physical constraint. This restriction of intramolecular rotations (RIR) blocks the non-radiative pathway and opens up the radiative channel. As a result, the HPS molecules become emissive in the aggregate state. ${ }^{29}$

According to our proposed mechanism, the RIR process is responsible for the AIE effect. We have conducted a number of control experiments (decreasing temperature, increasing viscosity, applying pressure, etc.) to externally activate the RIR process. We have also used covalent bonds to fasten the aryl rotors to internally set off the RIR process at the molecular level. In response to both the external and internal controls, the luminogens become emissive, thus offering experimental evidence to support our mechanistic hypothesis. ${ }^{29}$ Guided by the mechanistic understanding, our group has synthesized different kinds of luminogenic molecules with a structural feature of one conjugated stator carrying multiple aromatic peripheral rotors, such as polyarylated ethenes, butadienes, pyrans, fulvenes and arylenes. ${ }^{22-26}$ All the luminogens show AIE activity, with emission colour covering the entire visible region and luminescence efficiency reaching unity.

Besides our group, many other research laboratories have utilized the RIR rational to develop new AIE systems, which will be delineated in the following sections. Because of the fast progress in the area of AIE research, it is impossible for us to include all of the AIE luminogens reported so far in this review. Therefore, we will not elaborate on very detail but choose some typical examples to illustrate the working principles involved. The examples will be grouped together according to their structural features-hydrocarbon, heterocyclic, supramolecular, polymeric and organometallic. The "eccentric" systems without conventional chromophores will be introduced as an emerging group of new AIE luminogens.

\subsection{Hydrocarbon luminogens}

$\mathrm{Wu}$ et al. observed marked AIE effects for a series of 8,8 a-dihydrocyclopenta $[a]$ indene derivatives. ${ }^{30}$ As seen from the example shown in Fig. 2, 1 is non-luminescent when it is molecularly dissolved in pure THF. Addition of a large amount of water into its THF solution causes molecules of $\mathbf{1}$ to aggregate and luminesce. While the fluorescence quantum yield $\left(\Phi_{\mathrm{F}}\right)$ of the THF solution of $\mathbf{1}$ is virtually nil $(0.16 \%)$, the $\Phi_{\mathrm{F}}$ value of its aggregates suspended in the $\mathrm{THF} /$ water 

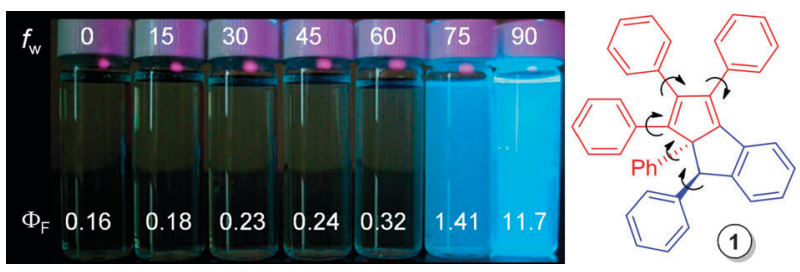

Fig. 2 Fluorescence photographs of the solutions or suspensions of the molecules or nanoaggregates of $\mathbf{1}$ in $\mathrm{THF} /$ water mixtures with different volumetric fractions of water $\left(f_{\mathrm{w}}, \mathrm{vol} \%\right)$; fluorescence quantum yields $\left(\Phi_{\mathrm{F}}, \%\right)$ of $\mathbf{1}$ estimated using quinine sulfate as standard. Reproduced with permission from ref. 30. Copyright (2008) Wiley-VCH.

mixture containing $90 \%$ of water is 73 -fold higher. Clearly, the photoluminescence (PL) behaviour of $\mathbf{1}$ is typical of an AIE luminogen.

Structurally $\mathbf{1}$ is similar to HPS but compositionally they are different: 1 contains no atoms other than carbon and hydrogen and is thus a pure hydrocarbon luminogen. It is a neutral molecule and experiences no donor (D)-acceptor (A) interaction, for it carries neither $\mathrm{D}$ nor $\mathrm{A}$ group. Wu et al. rationalized that the RIR process was at work in this AIE system. In the THF solution, the multiple phenyl pendants of $\mathbf{1}$ undergo active twisting motions against its 8,8a-dihydrocyclopenta $[a]$ indene core. The intramolecular motions serve as a non-radiative channel for its excited states to decay. In the aggregates, the intramolecular rotations are restricted or the RIR process is activated, thus turning on the emission of the luminogen. ${ }^{31}$

Compared with cyclic polyenes such as HPS and 1, linear polyenes enjoy richer structural variety and readier synthetic accessibility, permitting more systematic and in-depth study. 1,4-Distyrylbenzene, for example, is a linear polyene. Its all trans form (2; Fig. 3) is an ACQ luminophore: it is strongly and weakly fluorescent in the solution and aggregate states, respectively. ${ }^{32}$ When the $\alpha$-olefinic hydrogen atoms of $\mathbf{2}$ are replaced by methyl groups, its PL behaviour is changed: its methylated derivative (3) shows AIE activity, as reported by Diau, Hsu and co-workers. ${ }^{33}$ It is amazing that an outwardly minor structural variation elicits such a big alteration in the photophysical property, that is, a complete reversion in the light-emitting process or pathway.

Inspection of the crystal structures of $\mathbf{2}$ and $\mathbf{3}$ reveals two very different packing modes. ${ }^{34}$ The flat conformation of the molecules of $\mathbf{2}$ allows overlap packing (Fig. 3, left panel). In the face-to-face packing structure, the molecules experience

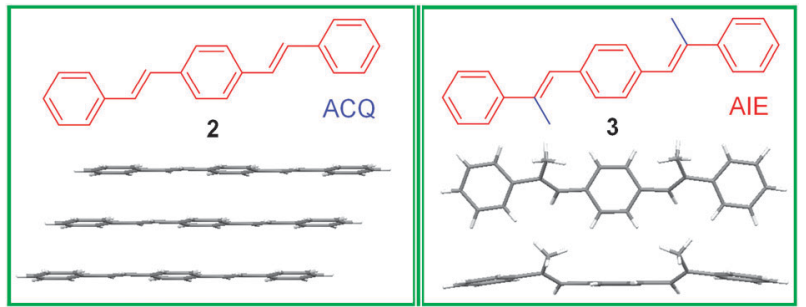

Fig. 3 Molecular packing arrangements of 1,4-di[(E)-styryl]benzene (2) and its $\alpha$-dimethylated derivative (3). Reproduced with permission from ref. 33. Copyright (2005) American Chemical Society. strong $\pi-\pi$ stacking interactions, which facilitates excimer formation and weakens light emission. ${ }^{35}$ Due to the steric hindrance of the two methyl groups, 3 adopts a non-planar conformation: its $\mathrm{C}-\mathrm{C}$ double bonds are twisted out of the central plane by $36.8^{\circ}$ and the torsional angle between the double bond and the flanking benzene ring is $>30^{\circ}$. This twisted geometry of $\mathbf{3}$ weakens its intermolecular interaction and minimizes the likelihood of excimer formation in the crystal. The RIR process activated by the crystal formation, on the other hand, makes $\mathbf{3}$ highly emissive in the solid state.

In the dilute solution, the molecules of $\mathbf{2}$ are isolated or segregated by the solvent molecules. The rod-like molecules of $\mathbf{2}$ enjoy a maximal extent of $\pi$-electron delocalization and can thus luminesce efficiently. The non-planar conformation of $\mathbf{3}$, however, imposes little bonding on its building blocks but allows active intramolecular rotations, which effectively deactivates its excitons non-radiatively, thereby rendering its molecules non-emissive in the solution state.

It becomes clear that the steric hindrance of the methyl groups of $\mathbf{3}$ has affected its molecular geometry, packing structure, molecular interaction and fluorescence behaviour. Such steric effect has been utilized in the design of other linear polyene luminogens. Through structural tailoring, a variety of arylenevinylenes with sterically imposed non-planarity have been developed, examples of which are given in Chart 2 . Substitution of $\alpha$ - or $\beta$-hydrogen atoms of 2 by phenyl rings works as efficiently as methyl groups: like $\mathbf{3}$, both $\mathbf{4}$ and $\mathbf{5}$ exhibit AIE activity, regardless of whether the phenyl substituents are located at the $\alpha$ - or $\beta$-position. ${ }^{36}$ The AIE activity is also observed in the system of $\mathbf{6}$, which is the $c$ is isomer of 5. This indicates that the AIE property is not determined by the conformation of its olefin unit but the propeller shape of its molecular structure.

In comparison to its trans congener, a cis isomer usually shows weaker luminescence in the solution state owing to its poorer electronic conjugation. A cis vinylene unit has thus been deemed a "cis defect" in a conjugated polymer chain, e.g., poly(phenylenevinylene). Ma et al. have found that 2,5-diphenyl-1,4-distyrylbenzene with a cis structure (7) emits

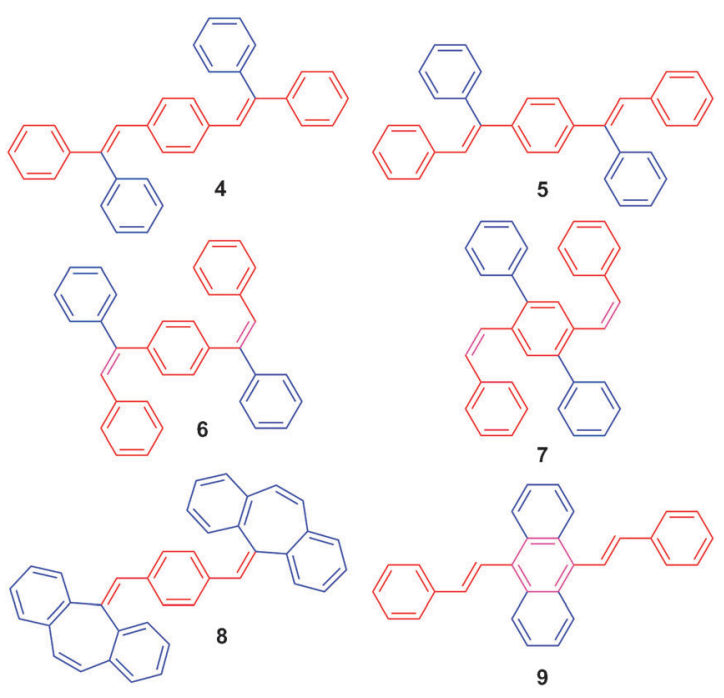

Chart 2 Examples of arylenevinylene-based AIE luminogens. 
a strong blue light in the crystalline state, although it is nonfluorescent in the dilute solution. ${ }^{37}$ The structure of the cis-isomer is significantly deviated from planarity, which makes it impossible for its molecules to pack in a co-facial way in the crystallization process. The RIR process activated by the crystal formation, however, makes the molecules of 7 highly emissive in the solid state.

Another molecular engineering strategy for transforming 1,4-distyrylbenzene (2) from a traditional ACQ luminophore to an AIE luminogen is to use large aromatic rings to replace the phenyl rings in the terminals or core of $\mathbf{2}$, because the steric effect of bulky aryl rings may induce conformational twists in the resultant luminogen. Examples of successful exploration along this line of structural design include the arylenevinylene luminogens containing big dibenzosuberene terminals (8) and anthracene cores (9). ${ }^{38,39}$ Both of these two luminogens are non-planar in conformation and propeller-like in shape and show marked AIE effects.

\subsection{Heteroatom-containing luminogens}

Pure hydrocarbon AIE systems are simple in structure and easy to synthesize and can serve as elementary models to elucidate the structure-property relationships involved in the AIE processes. The emissions from pure hydrocarbon AIE luminogens, however, are often blue in colour. To enrich the AIE palette and to widen the scope of high-tech applications of AIE luminogens, their emission colours need to be red-shifted, ideally with full coverage of the whole visible spectral region. Generally, the emission colour of a luminophore can be red-shifted by increasing its conformational planarity and electronic conjugation. Doing so, however, also increases intermolecular interactions and promotes the formation of such detrimental species as excimers and exciplexes, leading to the wicked ACQ effect.

Incorporation of heteroatoms into a luminogen can induce electronic perturbation, such as polarization caused by intramolecular charge transfer (ICT). This can considerably alter photophysical behaviours of the luminogen, particularly its colour tunability. ${ }^{40}$ Much work has been done along this line of consideration, an example of which is Tao's pyridinium salts, an AIE system with beyond-blue light emission. The dependence of PL intensity of such a pyridinium salt (10) on the solvent composition is shown in Fig. $4 .^{41}$ Because $\mathbf{1 0}$ is ionised, it is soluble in polar solvents (e.g., acetonitrile) but immiscible with non-polar solvents (e.g., toluene). In the solvent mixtures with "small" fractions of toluene $\left(f_{\mathrm{t}}\right), \mathbf{1 0}$ is non-emissive. In the mixture with $f_{\mathrm{t}}>75 \%$, its emission is intensified swiftly with increasing toluene content. Clearly, 10 is an AIE luminogen.

The AIE luminogen emits in the long wavelength region due to the polarization effect caused by the $\mathrm{D}-\mathrm{A}$ interaction between its amine and pyridinium units. As shown by the inset in Fig. 4, the powders of $\mathbf{1 0}$ emit a yellow light, whilst its dilute aqueous solution is non-fluorescent. In the dilute solution, the enantiomerization process of the Tröger's base unit (coloured in red in Fig. 4) and intramolecular motion of the styrylpyridinium salt units (coloured in blue) are active, which offer non-radiative channels for the excitons to relax.

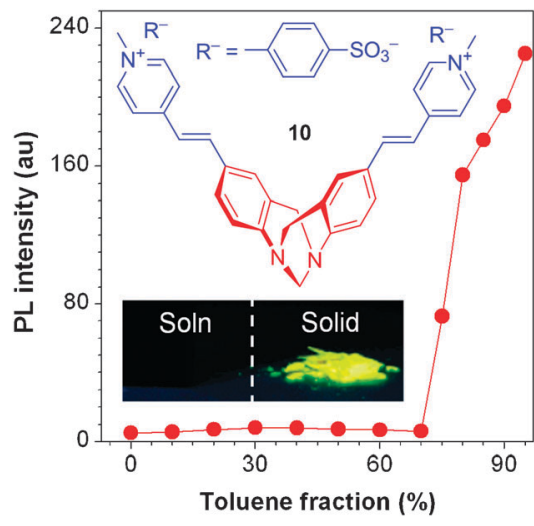

Fig. 4 Plot of fluorescence intensity of $\mathbf{1 0}$ versus solvent composition of acetonitrile/toluene mixture. Inset: photographs of an aqueous solution (Soln) of $\mathbf{1 0}$ and its powdery sample (Solid) taken under illumination of a UV lamp. Reproduced with permission from ref. 41. Copyright (2008) American Chemical Society.

These processes, however, are restricted in the aggregates. The non-planar Tröger's base unit barricades intermolecular interaction and frustrates excimer formation, thus activating the light-emitting process of $\mathbf{1 0}$ in the solid state.

More examples of heteroatom-containing luminogens are shown in Chart 3. Luminogens $\mathbf{1 1}$ and $\mathbf{1 4}$ emit blue light in the aggregate state, due to the absence of D-A interaction in the systems. ${ }^{42,43}$ Although triphenylamine (TPA) itself is not AIE active, its propeller-like structure and electron-donating attribute make it a useful building block for the construction of AIE luminogens, whose emission colours can be tuned through judicious combinations of the TPA units with other electron-accepting groups. This is demonstrated by the AIE luminogens containing the D-A pairs of TPA-benzaldehyde (12) and TPA-fluorenone (13), which emit green and red lights, respectively, due to the different extents of push-pull interactions involved in the two systems. ${ }^{44,45}$

In addition to high luminescence efficiency, high thermal stability is another perquisite for a luminogen to realize its device applications. Heteroatoms can also be used to enhance the stability of conjugated molecules. A series of carbazolecontaining AIE luminogens (e.g., 11) with high thermal stability have been developed by $\mathrm{Chi}, \mathrm{Xu}$, et al. ${ }^{42}$ The high stability of 11 is evinced by its high temperatures for glass transition $\left(T_{\mathrm{g}}=144{ }^{\circ} \mathrm{C}\right)$ and decomposition $\left(T_{\mathrm{d}}=488^{\circ} \mathrm{C}\right)$, noting that

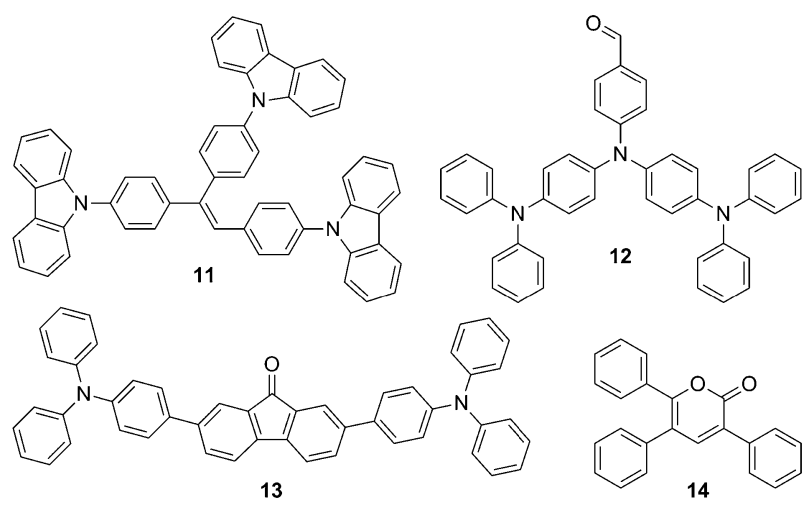

Chart 3 Examples of nitrogen- and oxygen-containing AIE luminogens. 


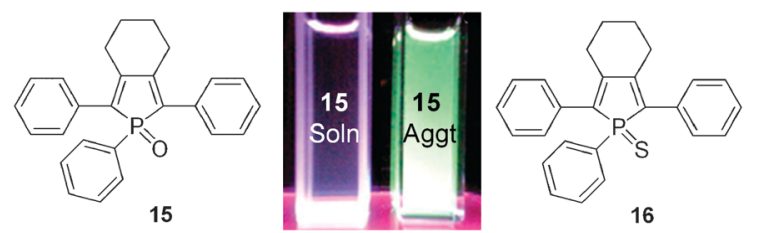

Fig. 5 Examples of phosphole luminogens. Photographs of $\mathbf{1 5}$ in THF (Soln) and THF/water (1:9v/v) mixture (Aggt) taken under illumination of a handheld UV lamp. Reproduced with permission from ref. 47. Copyright (2009) The Royal Society of Chemistry.

the $T_{\mathrm{g}}$ value for its pure hydrocarbon cousin $4,4^{\prime}$-bis(2,2diphenylvinyl)-1, $1^{\prime}$-biphenyl is as low as $64{ }^{\circ} \mathrm{C}$.

Phospholes are a group of unique heterocyclics that show properties not readily accessible by pyrroles and thiophenes. The phosphorus centre enables ready property modulation of phospholes by simple reactions, such as oxidation and metal complexation. ${ }^{46}$ Like HPS, phosphole oxide $\mathbf{1 5}$ and sulphide $\mathbf{1 6}$ possess a propeller structure. The AIE effect of $\mathbf{1 5}$ is manifested by the photographs shown in Fig. 5: faint and bright fluorescence are observed in its solution in THF and aggregate suspension in an aqueous mixture, respectively. ${ }^{47} \mathrm{As}$ reported by Réau et al., the $\Phi_{\mathrm{F}}$ value for a film of $\mathbf{1 6}$ is 10 fold higher than that for its dichloromethane solution. ${ }^{48}$

Benzobis(thiadiazole) (BTA) is a nitrogen- and sulphurcontaining heterocyclic compound well-known for its strong electron-withdrawing ability. The BTA unit has been widely used to tune optical properties of functional materials. Wang et al. have fused BTA and TPA units at the molecular level and generated an AIE luminogen (17; Chart 4) with a PL peak in the near-IR region. ${ }^{49}$ Thanks to the D-A pair of TPA-BTA, 17 exhibits a strong ICT effect and a low band gap energy. Due to its propeller-shaped TPA units, $\mathbf{1 7}$ adopts a twisted molecular conformation. Similar to the abovementioned AIE systems, the AIE effect of $\mathbf{1 7}$ has been rationalized to stem from the suppression of the $\pi-\pi$ stacking interaction and the activation of the RIR process in the aggregate state.

\subsection{Luminogens with cyano substituents}

Because of its structural simplicity and high polarizability, the cyano group has been frequently utilized as a functional unit in the design of advanced optical materials. As a result, a great number of AIE luminogens containing cyano groups have been developed. ${ }^{50-52}$ Although structurally they belong to the large family of heteroatom-containing luminogens, we sort them out and discuss them separately in this subsection, due to their unique behaviours and properties.

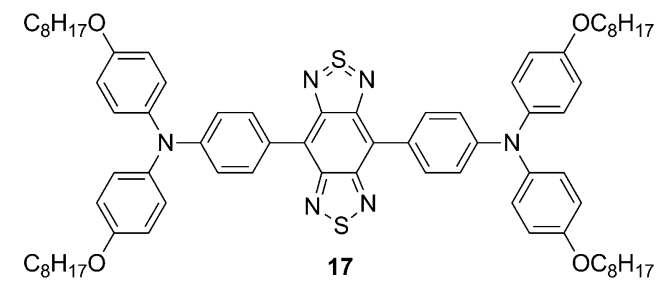

Chart 4 An example of benzobis(thiadiazole)-based near-infrared AIE luminogen.
Both steric and electronic effects of a cyano group affect the emission process of an AIE luminogen. The steric effect of a cyano group on the conformation of an AIE luminogen can be readily appreciated from the data shown in Fig. $6 .{ }^{53}$ The molecule of $\mathbf{1 8}$ takes a twisted conformation, owing to the steric repulsion between the cyano substituents and phenyl rings, noting that its parent form 1,4-distyrylbenzene (2) is planar in conformation ( $c f$., Fig. 3). In a dilute solution, the twisted geometry of $\mathbf{1 8}$ allows intramolecular rotation, which annihilates its excitons non-radiatively. In the crystals, such molecular motions are restricted. The $\mathrm{C}-\mathrm{H} \cdots \mathrm{N}$ hydrogen bonds formed between the cyano groups of one molecule and the hydrogen atoms of an adjacent molecule further rigidify the molecules and intensify their light emissions.

While the steric effect of the cyano group endows a distyrylbenzene derivative with AIE activity, its electronic effect enables the development of AIE systems with great colour tunability. Chen et al. ${ }^{54}$ and Neckers et al. ${ }^{55}$ have prepared a variety of diphenylfumaronitrile-type luminogens, examples of which are shown in Fig. 7. The colours of the emissions from the crystals or aggregates of luminogens $\mathbf{1 9}$ can be readily tuned from purple to blue to green and finally to red by simply changing the functional substituents on the phenyl rings. An obvious trend is observed in this AIE system: the emission colour is red-shifted with increasing electron-donating power of the substituent (19a $\rightarrow$ 19d), again proving the importance of the D-A interaction to the development of redder light emitters.

Photoresponsive materials have drawn much attention due to their controllable photonic properties. Azobenzene is the best-known photosensitive molecule. Its derivatives usually do not emit due to the photoinduced cis-trans isomerisation. Han and Hara, however, have reported a novel azobenzene derivative (20). Upon UV irradiation, its non-emissive trans form undergoes trans-cis isomerization, accompanied with a large enhancement in the light emission (Chart 5). ${ }^{56}$ The PL enhancement originates from aggregate formation, as proved by the fluorescence microscope study. The bent shape of $c i s-\mathbf{2 0}$ is more hydrophilic, relative to its rod-shaped trans form. The amphiphilic molecules of cis-20 naturally self-assemble into spherical aggregates with the aid of the hydrophobic interaction between the long alkyl chains. The polar cyano

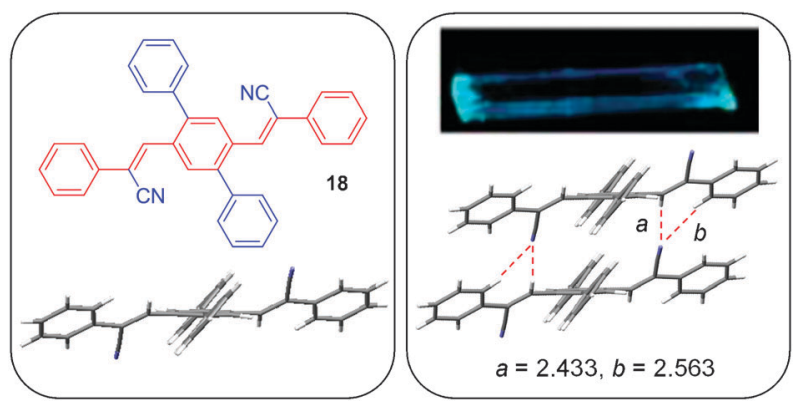

Fig. 6 Steric effect of cyano substituents on the molecular geometry and crystal packing of arylenevinylene luminogen 18. Photograph of a piece of crystal of $\mathbf{1 8}$ taken under UV illumination. The C-N $\cdots H$ hydrogen bonds in the crystal packing structure of $\mathbf{1 8}$ are marked by dotted lines. Reproduced with permission from ref. 53. Copyright (2007) The Royal Society of Chemistry. 

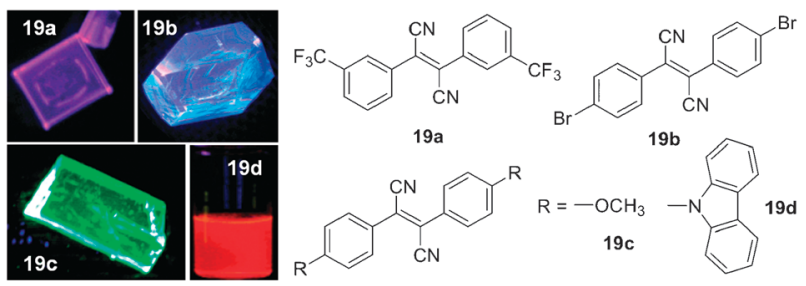

Fig. 7 Photographs of crystals of diphenylfumaronitrile derivatives 19a-19c and nanoaggregates of $19 d$ suspended in a THF/water mixture $(1: 9 v / v)$ taken under illumination of a handheld UV lamp. Reproduced with permission from ref. 54 [Copyright (2004) American Chemical Society] and ref. 55 [Copyright (2008) American Chemical Society].

group may have played an important role in facilitating the head-to-tail arrangement of the chromophoric units and in promoting the formation of $\mathrm{J}$-type aggregates.

\subsection{Luminogens with hydrogen bonds}

Hydrogen bonding is an important non-covalent force often involved in the formation of supramolecular structures in the solid state. ${ }^{57-59}$ In the area of photophysics research, it has been generally recognized that formation of hydrogen bonds between luminogens can rigidify their molecular structures and activate their RIR processes. This helps minimize the nonradiative energy losses of their excitons and maximize their probability of radiative transitions. A nice example that manifests the importance of hydrogen bonding to the lightemitting process is the excited-state intramolecular proton transfer (ESIPT).

Dibenzalazine does not fluoresce in the solution and solid states. Its hydroxylated derivative $N, N^{\prime}$-disalicylalazine (21; Chart 6), however, emits strongly in the crystalline state, although it is non-emissive in the solution state. ${ }^{60}$ The $o$-hydroxyl groups in $\mathbf{2 1}$ allow the formation of intramolecular hydrogen bonds in the excited state, a process known as the ESIPT. ${ }^{61}$ In the solution state, the dynamic intramolecular rotations of the phenyl rings around the $\mathrm{C}-\mathrm{C}$ and $\mathrm{N}-\mathrm{N}$ axes non-radiatively extinguishes the excited states of 21. In the crystalline state, however, the rigid environment favours the formation of hydrogen bonds, which stiffens the structure of the luminogen and turns on its light emission.

More examples of ESIPT luminogens are presented in Chart 6. Li, Yang, et al. found that nanoparticles of 22 were more emissive than its solutions. ${ }^{62}$ Araki et al. accomplished switchable emission of $\mathbf{2 3}$ by thermal manipulation of its

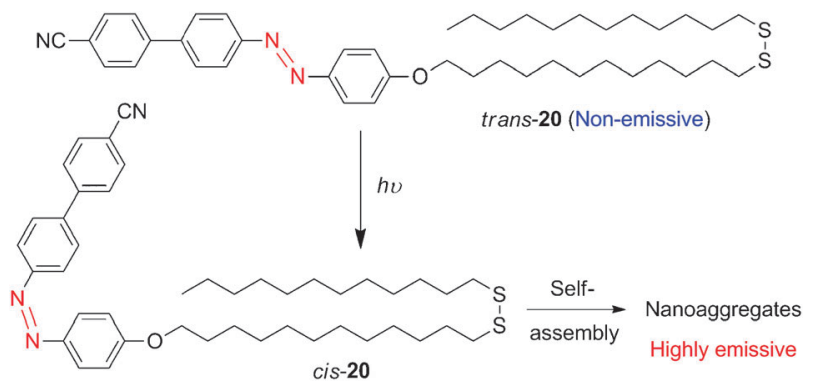

Chart 5 Effect of photoinduced trans-to-cis isomerization on the light-emitting behaviour of azobenzene-containing luminogen $\mathbf{2 0}$.

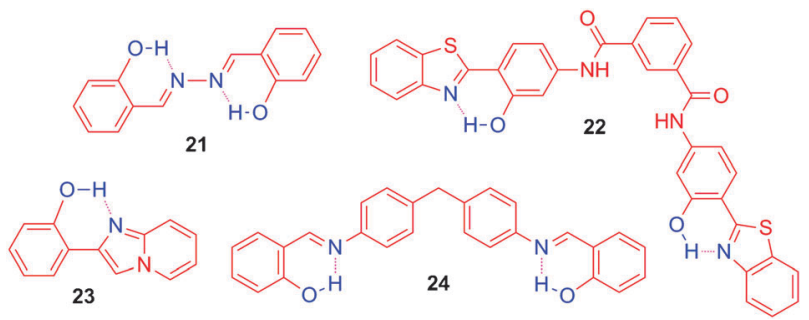

Chart 6 Examples of AIE luminogens with their molecular structures stiffened by intramolecular hydrogen bonds in the aggregate state.

molecular packing in the solid state. ${ }^{63}$ In 2006, Birkedal and Pattison found that the V-shaped molecules of $\mathbf{2 4}$ could pack into J-aggregates with enhanced emission. ${ }^{64}$ Later, Lu et al. prepared an organogelator based on a derivative of $\mathbf{2 4}$ with cholesteryl substituents. ${ }^{65}$ Thanks to the chiral moieties, the luminogens self-assemble into 1D nanofibers, followed by further evolution into 3D networks in the gel state. The gel shows stronger emission than its solution. The AIE activities of these luminogens have been attributed to their RIR and ESIPT processes in the solid state.

Intermolecular hydrogen bonding plays a vital role in the gelling processes of many small-molecule gelators. In 2003, Park et al. reported gelation-induced emission of a benzene1,3,5-tricarboxamide derivative: its non-fluorescent solution became emissive upon gel formation. ${ }^{66}$ Bo et al. developed a similar system. ${ }^{67}$ Fig. 8 depicts the assembly process of $\mathbf{2 5}$ consisting of a terphenylene core and two dendritic wedges. With the aid of the synergistic effect of the non-covalent forces, including intermolecular hydrogen bonding and hydrophobic interactions, an interpenetrating 3D network or gel is formed. The intramolecular rotations are largely restricted in the gel, which populates the radiative transitions and enhances the light emissions.

Lu et al. developed a series of binary supramolecular gel systems, an example of which is sketched in Chart $7 .^{68}$ Although the molecules of $\mathbf{2 6}$ themselves cannot assemble into supramolecular structures, they readily form organogels in the presence of L-tartaric acid. The multiple binding sites

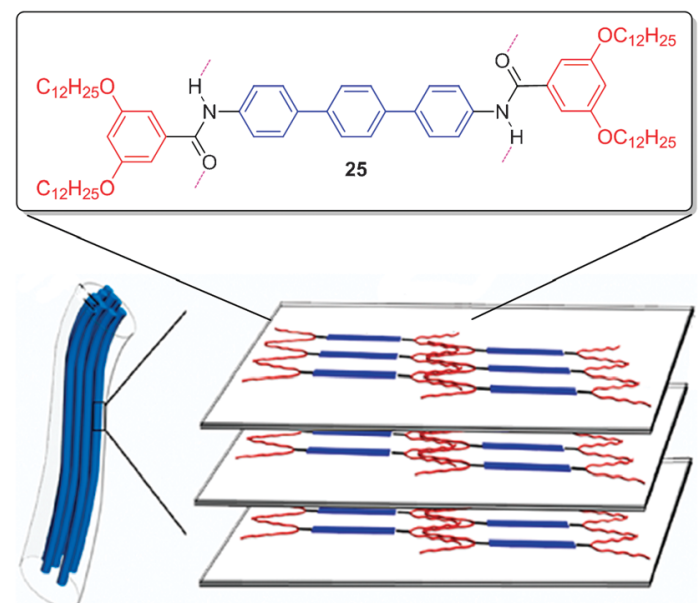

Fig. 8 Diagrammatic representation of possible packing arrangement of luminogen molecules of $\mathbf{2 5}$ in the gel state. Reproduced with permission from ref. 67. Copyright (2009) American Chemical Society. 


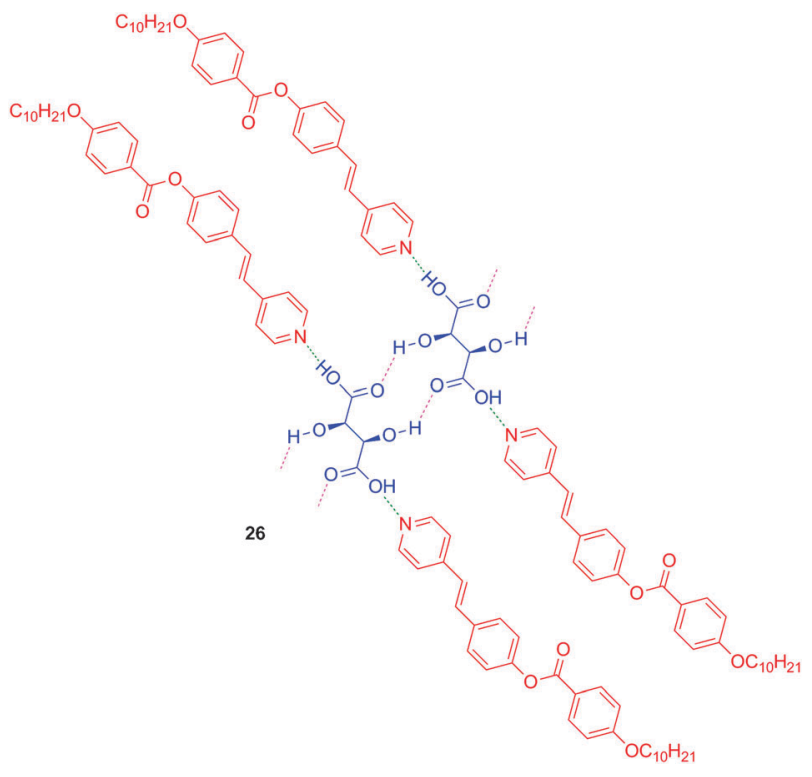

Chart 7 Schematic illustration for supramolecular complexation of 26 with L-tartaric acid through the formation of multiple hydrogen bonds.

offered by L-tartaric acid enable the gelling process to occur through the formation of intra- and intermolecular hydrogen bonds. The organogels emit brightly, due to the activation of the RIR process by the hydrogen-bond-aided supramolecular self-assembly.

\subsection{Polymeric luminogens}

In the supramolecular assembly processes discussed above, the building blocks are connected by non-covalent linkages. As such, the luminescent properties of the assemblies can be readily and reversibly tuned by external stimuli. The loose connections by the weak forces, however, provide the supramolecular assemblies with little mechanical strengths. Small molecule luminogens, on the other hand, often need to be processed by expensive techniques, such as vacuum vapour deposition. One way to surmount the mechanical weakness and processing disadvantage is to synthesize high molecular weight polymers, which can be readily fabricated into largearea, mechanically tough films by simple processes, such as spin-coating and static-casting. ${ }^{69}$

A series of conjugated polymers that show enhanced light emissions in the solid state have been developed in recent years, examples of which include poly(1-phenyl-1-alkyne)s (27) and poly(diphenylacetylene)s (28; Chart 8). ${ }^{69,70}$ These polymers fluoresce when dissolved and emit more intensely when aggregated, showing a phenomenon of aggregationenhanced emission (AEE). A common structural feature for these polymers is that each of their repeat units contains phenyl rotor(s) and an olefin stator. ${ }^{69,70}$ This is similar to tetraphenylethene (TPE), a well-known AIE luminogen. ${ }^{29}$ In TPE, its olefin stator is surrounded by phenyl rotors and the RIR process is known to account for its AIE effect. In the polymer systems, the intramolecular rotations of the phenyl rotors are already partly restricted at molecular level due to the steric effect of polymer chains. The polymers are thus fluorescent in the solution state, which explains why they are not AIE but AEE active.

Another approach to AEE-active polymers is to use AIE luminogens as monomers. ${ }^{71,72}$ For example, 29 (Fig. 9) is a polymer constructed from knitting up TPE luminogens by vinylene linkages. While TPE is totally non-emissive in the solution state, the solution of $\mathbf{2 9}$ is fluorescent, although its intensity is low. Its emission is greatly enhanced when its molecules are aggregated, confirming its AEE nature. ${ }^{70}$ AEE effects have been frequently observed in polymer systems, because the intramolecular rotations become more difficult when the luminogens are hooked up by a polymer chain.

Intriguingly, 29 shows aggregation-enhanced two-photonexcited fluorescence (TPEF): the TPEF intensity of its nanoaggregates is 20-fold higher than that of its solution (Fig. 9). When the polymer chains are induced to aggregate, a large increase in the two-photon absorption cross-section $(\sigma)$ is observed. Its solution and aggregates show $\sigma$ values of 107 and $896 \mathrm{GM}$, respectively, when excited at a wavelength of $750 \mathrm{~nm}\left(\lambda_{\mathrm{ex}}\right)$. The $\sigma$ value of its nanoaggregates is further increased to $1361 \mathrm{GM}$ at $\lambda_{\mathrm{ex}}=740 \mathrm{~nm}$. These $\sigma$ values are pretty high for a pure hydrocarbon luminogen containing no D-A units. Prasad et al. studied two-photon absorptions of their AIE luminogens: enhanced luminescence was observed for the hydrophobic aggregates in the aqueous media. ${ }^{32}$ The researchers successfully used the AIE luminogens for twophoton fluorescence cell imaging applications. ${ }^{73}$

Chujo's laboratory reported AIE phenomena of a group of polymers consisting of repeat units of $o$-carborane and p-phenyleneethylnylene. ${ }^{74}$ The boron cluster of carborane was chosen as a monomer due to its well-known 3D aromaticity. The carborane model compound 30 (Fig. 10) shows AIE activity, emitting an intense blue light in the solid state. The dihedral angle between the two phenyl rings attached to the carborane core is big $\left(58^{\circ}\right)$, which hampers the formation of intra- and intermolecular excimers. Polymerizations of the derivatives of $\mathbf{3 0}$ yield boron-containing polymers (e.g., 31; Fig. 10). Like its monomer, $\mathbf{3 1}$ is also AIE active. Its film emits an orange light, due to the extended conjugation along the polymer chain.
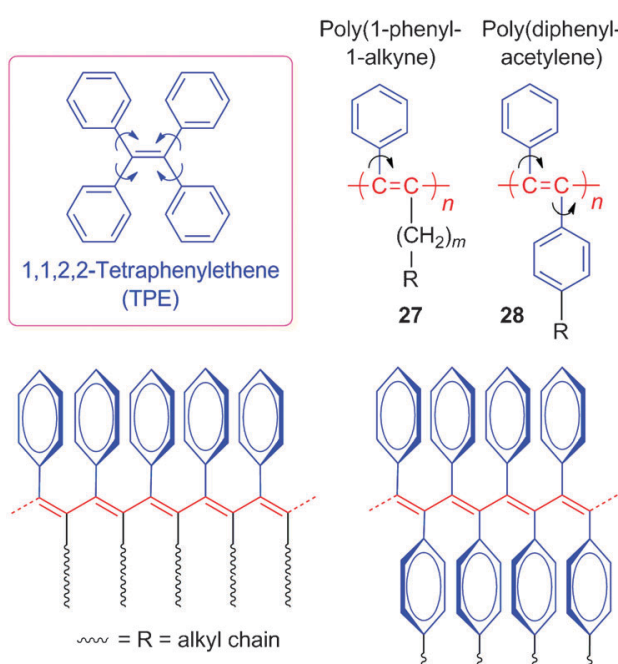

27

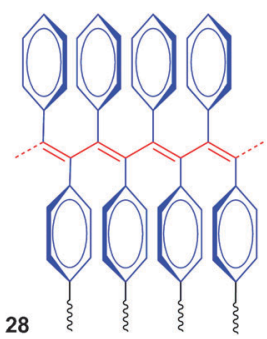

Chart 8 AIE-active TPE and AEE-active disubstituted polyacetylenes. 


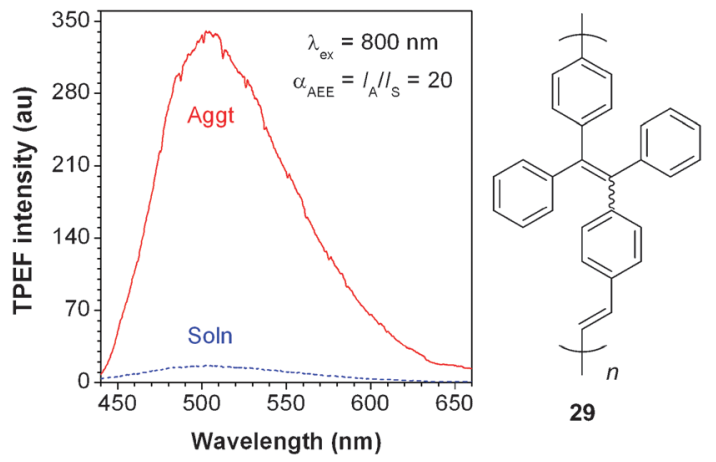

Fig. 9 Two-photon-excited fluorescence (TPEF) spectra for a solution (Soln) of $\mathbf{2 9}$ in THF $(2.6 \mu \mathrm{M})$ and its aggregates (Aggt) in a $\mathrm{THF} /$ water mixture (1:9 by volume).
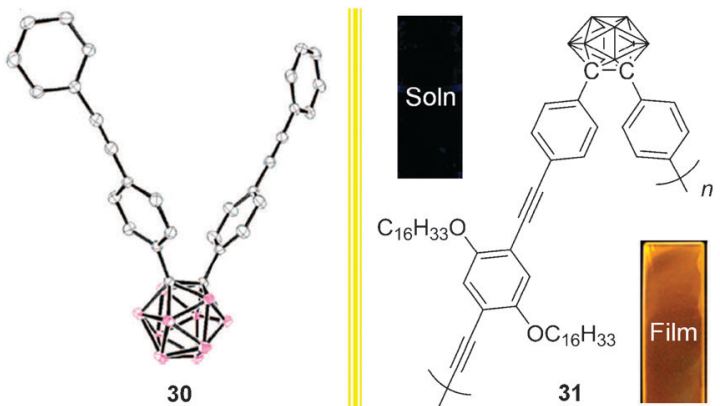

Fig. 10 (Left) Molecular structure of AIE luminogen of $o$-carborane 30. (Right) Chemical structure of carborane-containing polymer $\mathbf{3 1}$. Inset: fluorescence photographs of a solution of $\mathbf{3 1}$ and its film taken under illumination of a UV lamp. Reproduced with permission from ref. 74. Copyright (2009) American Chemical Society.

In addition to linear polymers, hyperbranched polymers with enhanced luminescence in the aggregate state have also been developed. ${ }^{75,76}$ For example, hyperbranched polysilole $\mathbf{3 3}$ was obtained from the polycyclotrimerization of silole-based diyne monomer 32 (Scheme 1) ${ }^{77}$ Polymer 33 shows AEE activity. Its silole units are knitted up by the newly formed benzene rings and located in a stiff polymer sphere. This limits the intramolecular rotations of its phenyl rotors against the silole stators to some extent, thus making $\mathbf{3 3}$ fluorescent in the solutions. The intramolecular rotations are further restricted by aggregate formation, which enables the polymer to emit more efficiently in the solid state. ${ }^{69}$

\subsection{Luminogens without orthodox chromophores}

In the synthesis of a TPE derivative with an unsymmetrical structure, we found that a by-product was constantly formed, which could be isolated by chromatographic separation. The by-product was easily crystallized, which allowed structural elucidation by crystallographic analysis. To our surprise, the by-product was found to be tetraphenylethane ( $s$-TPE), with four phenyl rings linked by $\mathrm{C}-\mathrm{C}$ single bonds (Fig. 11). We measured its emission spectra in the solution and aggregate states. Unlike its counterpart of TPE, both the solution of $s$-TPE in THF and its aggregates in a THF/water mixture with 90 vol $\%$ of water are virtually non-luminescent (Fig. 11A).

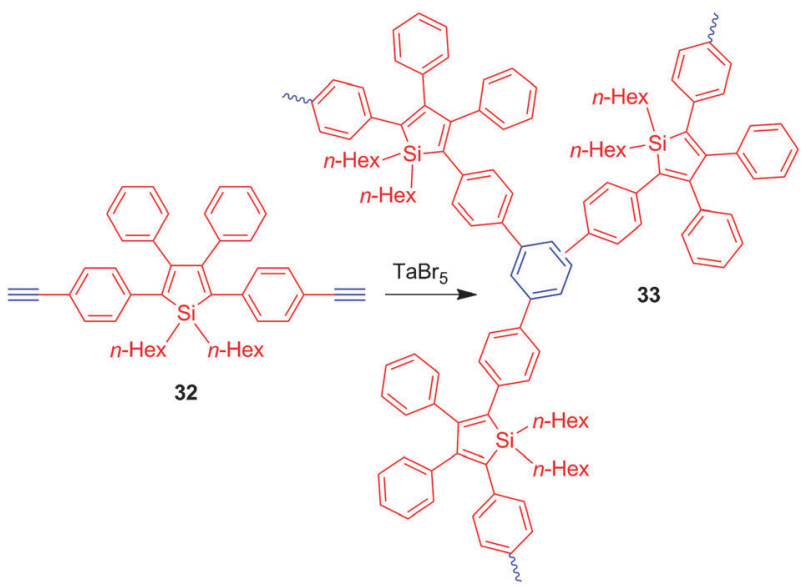

Scheme 1 Synthesis of silole-containing hyperbranched polyphenylene (33) by diyne polycyclotrimerization. Reproduced with permission from ref. 77. Copyright (2010) American Chemical Society.

This is understandable, because $s$-TPE is electronically nonconjugated.

Amazingly, however, the crystals of $s$-TPE emit intensely with an emission peak at $456 \mathrm{~nm}$ (Fig. 11), showing a novel phenomenon of crystallisation-induced emission (CIE). This indicates that $s$-TPE is a heterodox luminogen containing no canonical chromophore. Its CIE effect may stem from the intermolecular interactions of the $s$-TPE molecules in the crystals. Scrutiny of the packing patterns, however, found no salient $\pi-\pi$ stacking between the adjacent $s$-TPE molecules. The exact cause for the CIE effect of $s$-TPE is unclear at present. Time-resolved spectral analyses of its crystals gave a luminescence lifetime of a few nanoseconds, excluding the involvement of the triplet excited states in the PL process.

Another example of luminogens containing no classical chromophores was recently reported by Lehn et al. ${ }^{78,79}$ They created a new class of dynamic polymers (dynamers) with reversible connections of monomer units. For example, $\mathbf{3 4}$ was obtained from the polycondensation of bishydrazide and dialdehyde containing oligosaccharide moieties. Whereas its monomers are non-emissive in the solution and solid states, $\mathbf{3 4}$ emits an intense blue light even in the solution (Fig. 12). The polymer backbone may be rigidified by the bulky oligo-saccharide

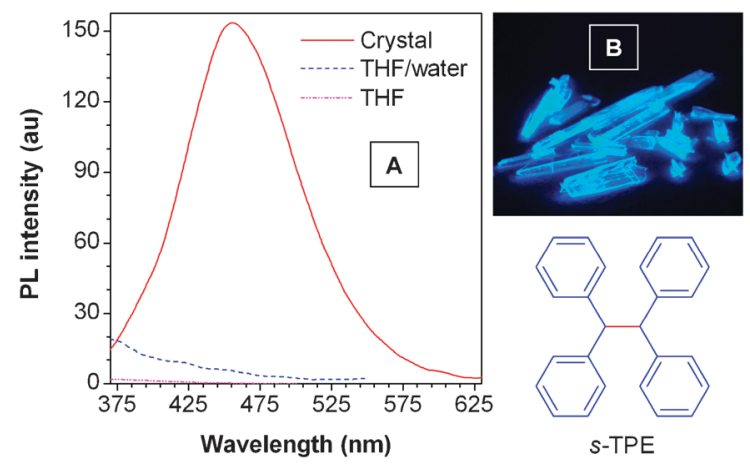

Fig. 11 (A) Emission spectra of the solution (in THF), aggregates (in 1:9 THF/water mixture) and crystals of 1,1,2,2-tetraphenylethane (s-TPE). (B) Photograph of its crystals taken under illumination of a UV lamp. 

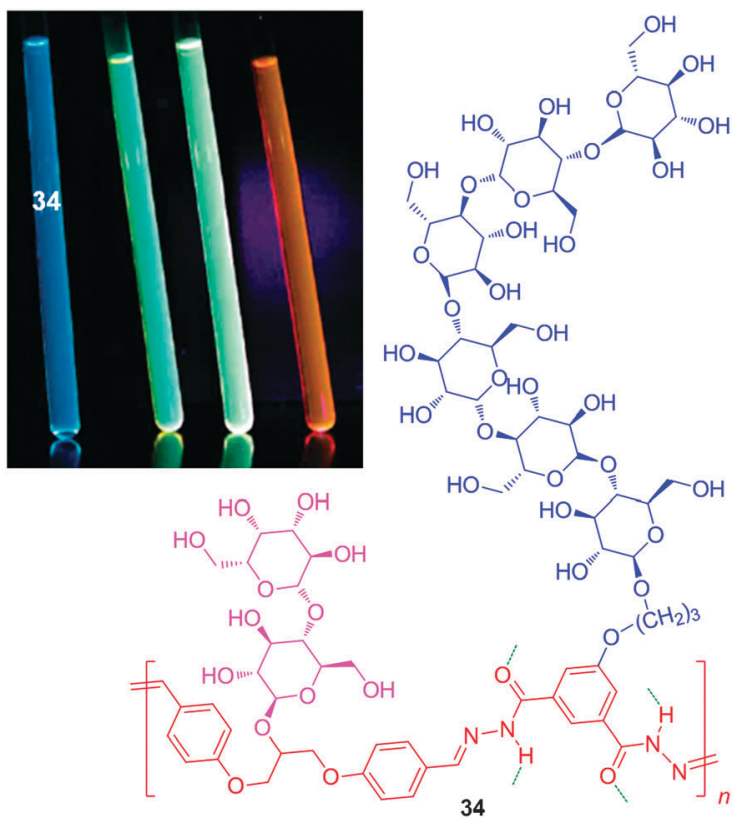

Fig. 12 Fluorescent photographs of glycodynamer $\mathbf{3 4}$ and its congeners with different constituents in $\mathrm{D}_{2} \mathrm{O}$ taken under illumination of a UV lamp. Reproduced with permission from ref. 78. Copyright (2010) American Chemical Society.

substituents and the polymer chains may be stiffened by the mutiple interchain hydrogen bonds, both of which activate the RIR process of the aromatic hydrazone units, thus making 34 emissive. The emission colour of the dynamers can be tuned by mixing with different monomers, between which dynamic exchange reactions take place. ${ }^{78}$

The AIE effect has been observed in some polymers even without phenyl rings. In 2007, we reported that the colloidal nanoparticles of poly[(maleic anhydride)-alt-(vinyl acetate)] (35) emitted strongly at $419 \mathrm{~nm}$, whereas its solution gave only faint emission (Fig. 13A) ${ }^{80,81}$ The PL intensity of the suspension of $\mathbf{3 5}$ is increased with an increase in the colloid loading (Fig. 13B). The luminescence lifetime of the nanoaggregates is in the nanosecond region, indicating that the emission is fluorescence in nature. Though the exact reason for the PL of the nanoparticles is unknown, it may be related to the molecular interactions between the five-membered dihydrofuran-2,5-dione rings, because the hydrolysis of the anhydride unit totally annihilates the light emission. Similar phenomenon has been observed by Pucci et al. for a polymer carrying succinic anhydride moiety (36): its solution is nonemissive but its aggregates is highly luminescent (Fig. 14). ${ }^{82}$

\subsection{Organometallic luminogens}

The above discussions are all on the emission enhancements observed in the organic luminogenic systems. The emissions are fluorescence in nature, because the radiative decays are associated with the transitions of singlet excited states. ${ }^{29,75}$ Aggregation-induced phosphorescence has been observed in the organometallic luminogen systems with emissions from triplet excited states. ${ }^{83-88}$ This widens the area of research to phosphorescence and manifests the omnipresence of the AIE effect.
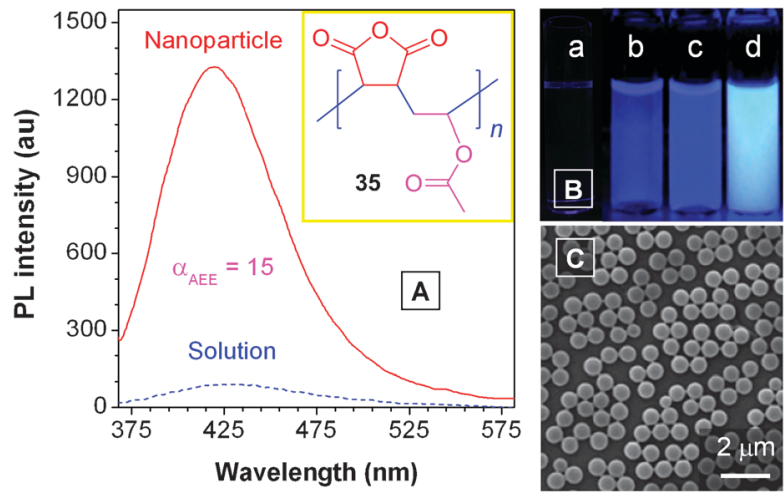

Fig. 13 (A) Emission spectra of nanoparticles of $\mathbf{3 5}$ suspended in butyl acetate and its solution in THF; [35 $]=0.5 \mathrm{wt} \%$. (B) Photographs of (a) THF solution and (b-d) nanoparticle suspensions of $\mathbf{3 5}$ taken under illumination of a UV lamp. Nanoparticle concentrations (wt\%): (b) 0.05, (c) 0.1, (d) 1.0. (C) SEM image of the nanoparticles. Reproduced with permission from ref. 80. Copyright (2007) American Chemical Society.

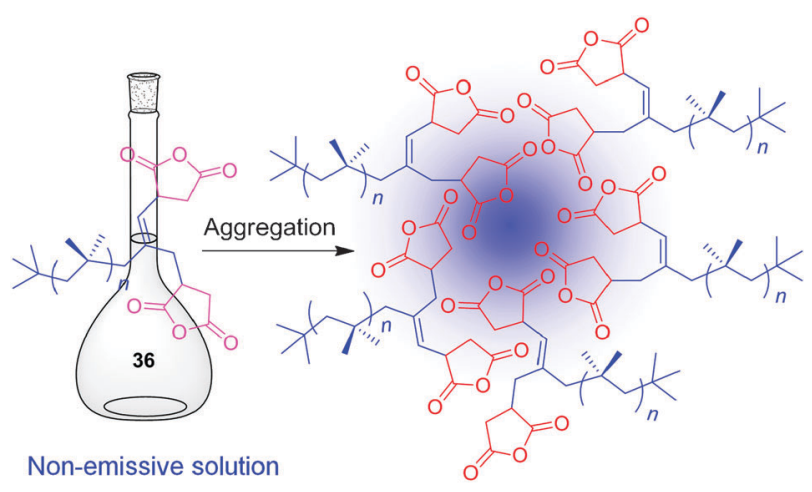

Emissive aggregates

Fig. 14 Effect of aggregation on the emission behaviour of polymer 36. Reproduced with permission from ref. 82. Copyright (2009) Wiley-VCH.

Che et al. reported that an acetonitrile solution of $\mathrm{Pt}(\mathrm{II})$ complex 37 (Chart 9) is virtually non-phosphorescent $\left(\Phi_{\mathrm{P}}=0.004\right) .{ }^{84}$ However, when a large amount of water is added into acetonitrile, the emission of $\mathbf{3 7}$ is enhanced by 7.5 times and red-shifted by $103 \mathrm{~nm}$. In the aqueous mixtures, the molecules of the Pt(II) complex form aggregates, which sets off the RIR process and boosts the phosphorescence. It is hypothesized that the intramolecular hydrophobic folding of the $o$-phenyleneethynylene chain of $\mathbf{3 7}$ is responsible for its solvatochromic phosphorescence. The similar phenomenon was observed by $\mathrm{Lu}$ et al. in the Re(I) complex systems. ${ }^{85}$ Large enhancements in the emission intensity and quantum yield of 38 were observed when the medium was changed from an organic solvent to an aqueous mixture.

Phosphorescent Ir(III) complexes have been extensively studied, many of which, however, are on their emission behaviours in the solution state. ${ }^{86} \mathrm{Li}$ et al ${ }^{87}$ and Park et al. ${ }^{88}$ reported aggregation-induced phosphorescence in a series of Ir(III) complex systems, although the cause of the effect is still under debate. ${ }^{87-89}$ Park's laboratory prepared a group of Ir(III) complexes containing imine-based ancillary ligands (Fig. 15). The complexes emitted faintly in the solution state 

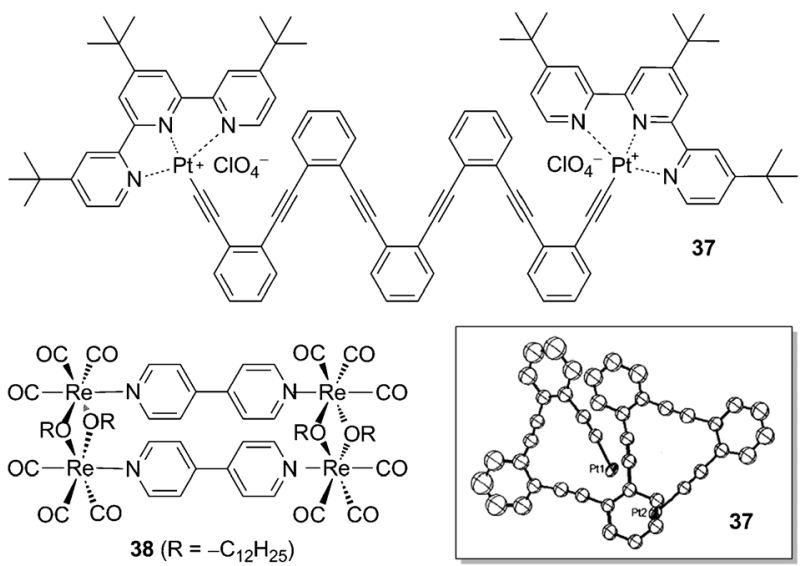

Chart 9 Examples of platinum (37) and rhenium (38) complexes with AIE activity and ORTEP drawing of partially folded conformation of 37 .

but brightly in the solid state, with a $>100$-fold increment in $\Phi_{\mathrm{P}}$. Strong phosphorescence was also observed at cryogenic temperatures, at which the solution of $\mathbf{3 9}$ was frozen. On the basis of the experimental results, the researchers concluded that the RIR process of the $\mathrm{N}$-aryl unit was the main cause for the observed AIE effect. ${ }^{88}$

\section{Mechanistic discussion}

In the previous section, we have presented various examples of different classes of AIE luminogens and briefly discussed their plausible working principles. Though the RIR process has been proposed to be the main cause for most of the reported AIE systems, detailed mechanistic paths need to be further elaborated. In this section, we will attempt to piece together the scraps of scattered information on the structure-property relationships reported by different research groups, in order to draw a clearer picture on the novel photophysical processes. Particular efforts will be made to learn how the structural aspects, such as $\pi$-conjugation, conformation and packing, influence the light-emitting behaviours of the AIE luminogens. On the basis of the mechanistic understanding, structural design strategies for transforming traditional ACQ luminophores to new AIE luminogens will be introduced and discussed.

\subsection{Planarity and rotatability}

According to fundamental physics, any movements, microand macroscopic alike, all consume energy. ${ }^{7,29}$ As discussed
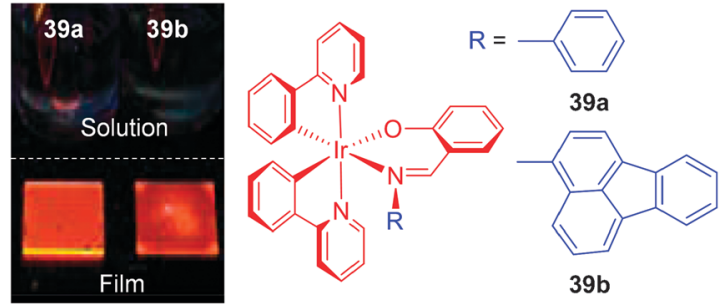

Fig. 15 Photographs of solutions and films of iridium(III) complexes 39 taken under illumination of a UV lamp. Reproduced with permission from ref. 88. Copyright (2008) The Royal Society of Chemistry. above, the intramolecular rotations of aromatic rotors in an AIE luminogen are active in the solution state, which serves as a relaxation channel for its excitons to non-radiatively decay. In the aggregate state, however, the intramolecular rotations are restricted due to the physical constraint, which blocks the non-radiative pathway and opens the radiative channel. Provided that this RIR hypothesis holds true, the question follows: why are some molecules emissive in the solution state, even though they contain seemingly readily rotatable aromatic rings?

The phenyl rings in $\mathbf{2}$ and $\mathbf{3}$, for example, appear to be equally rotatable but the two molecules show very different luminescence behaviours. ${ }^{33,90,91}$ In Fig. 3, we tried to use the crystal data to explain the difference. We take a theoretical approach here, in an effort to learn more about the emission processes. Computational optimizations show that 2 and 3 adopt planar and twisted conformations, respectively. The effects of the conformations on the molecular motions are shown in Fig. 16, where the reorganization energy is the energy required for structural adjustments. ${ }^{92}$ The highfrequency modes are related to the stretching vibrations of $\mathrm{C}-\mathrm{C}$ and $\mathrm{C}=\mathrm{C}$ bonds, while the low-frequency modes $\left(<100 \mathrm{~cm}^{-1}\right)$ are assigned to the torsional or twisting motions of the aromatic rings. ${ }^{92,93}$ The energy calculations indicate that the low-frequency modes occupy a large portion $(\sim 47 \%)$ of the total reorganization energies of $\mathbf{3}$, while the low-frequency modes are negligibly small in the case of 2 .

Shuai has conjectured that the low-frequency motions are associated with the non-radiative energy dissipations in the solution state. ${ }^{94}$ These motions are readily suppressed in the aggregate state or at low temperature, leading to recovery of the radiative transitions. This conjecture is further proved by the difference between the emission behaviours of $\mathbf{4 0}$ and $\mathbf{4 1} .^{91}$ Luminophore $\mathbf{4 0}$ takes a flat conformation and shows a solution quantum yield $\left(\Phi_{\mathrm{F}, \mathrm{s}}\right)$ as high as $80 \%$. Attachment of two phenyl rings to the double bond in the middle twists the

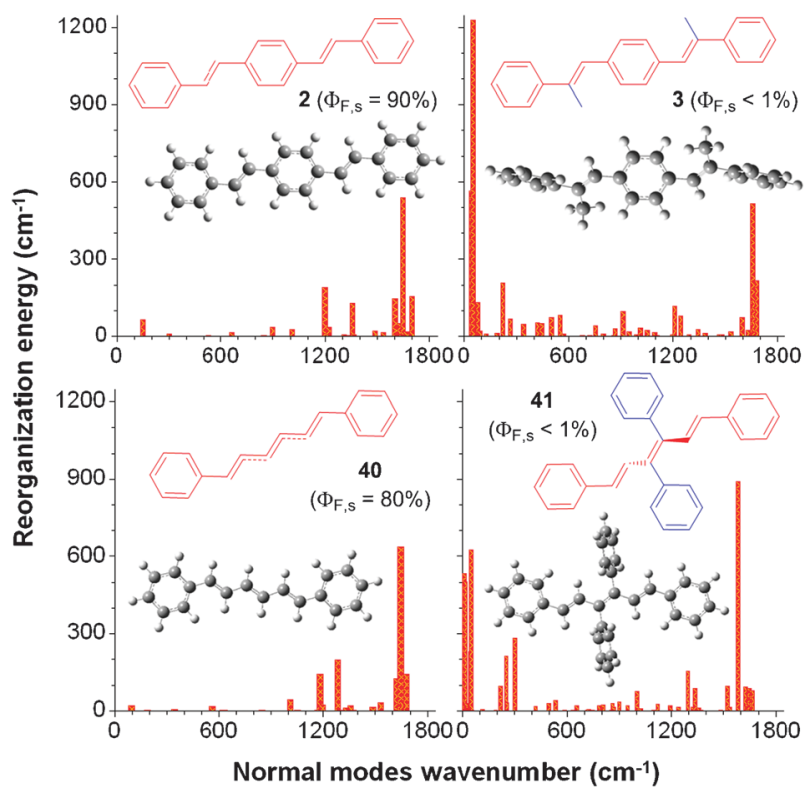

Fig. 16 Effect of structural planarity on molecular motions of polyenebased luminophores. 
conformation of $\mathbf{4 1}$ and turns off its emission in the solution state $\left(\Phi_{\mathrm{F}, \mathrm{s}}<1 \%\right)$. Here again, the energy calculations reveal that $\mathbf{4 0}$ and $\mathbf{4 1}$ show weak and intense low-frequency modes, respectively, indicating that the low-frequency motions are responsible for the emission quenching of $\mathbf{4 1}$ in the solution state.

On the basis of above discussions, we elaborated a simple model to correlate geometrical planarity and intramolecular motion with luminescence behaviour. Assume that two chromophore units A and B make up a luminescent molecule through the linkage of a rotatable bond (e.g., $\mathrm{C}-\mathrm{C}$ single bond). In this graphic model, $\psi, \theta$, and $\omega$ define the dihedral angle between units $\mathrm{A}$ and $\mathrm{B}$, the extent of intramolecular rotation/torsion, and the vibrational frequency, respectively (Fig. 17). In other words, $\psi$ is used to indicate the planarity of the molecule, whilst $\theta$ and $\omega$ specify its rigidity.

When the chromophoric units A and B are parallel to each other $\left(\psi \sim 0^{\circ}\right)$, molecule $\mathrm{A}-\mathrm{B}$ enjoys a maximal electronic conjugation and a minimal potential energy, as shown in the upper panel of Fig. 17. The extended cross-chromophore electronic delocalization endows the $\mathrm{C}-\mathrm{C}$ linkage with some double-bond character. This stiffens the conformation of the molecule and boosts its resistance to the perturbations by the intramolecular rotations. The chromophoric units may still swing in small amplitudes $(\theta)$ but the trifling low-frequency motions are insufficient to quench the PL process. The highfrequency $(\omega)$ vibrations thus become the major modes of molecular motions and the potential energy surface becomes steeper. Due to the conformation stiffness, the luminophore has small overall reorganization energies, which enables it to give a high $\Phi_{\mathrm{F}}$ value in the solution state.

On the other hand, if the two chromophoric units are twisted out of plane due to the steric effect, as shown in the lower panel of Fig. 17 for the case of $\psi^{\prime}>0^{\circ}$, the overlap between the $\pi$-electron clouds of the $\mathrm{A}^{\prime}$ and $\mathrm{B}^{\prime}$ plates becomes smaller and the cross-chromophore $\pi$-conjugation becomes weaker. The $\mathrm{C}-\mathrm{C}$ linkage between $\mathrm{A}^{\prime}$ and $\mathrm{B}^{\prime}$ units thus poses little restraint to their intramolecular rotations. Such motions require lower energy, in agreement with the larger contribution of the low-frequency $\left(\omega^{\prime}\right)$ modes to the calculated molecular motions ( $c f$., Fig. 16). The smaller $\omega^{\prime}$ values lead to a shallower potential energy surface, meaning that there is little energy barrier to a conformational change. The rotational amplitudes should be larger, for the $\theta^{\prime}$ values can

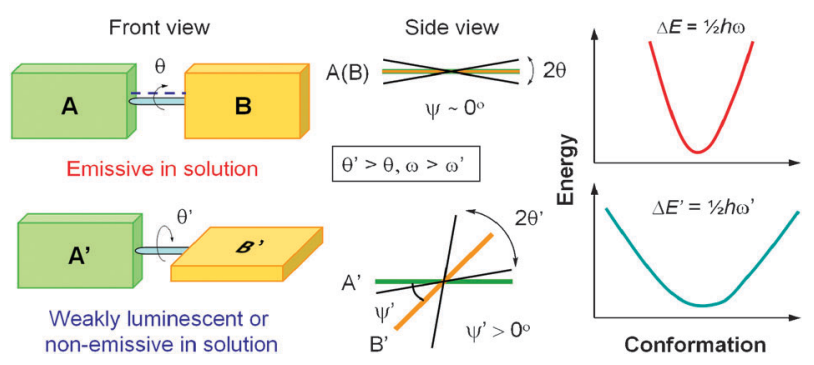

Fig. 17 Schematic illustration of the relationship among conformational planarity, torsional motion, energy surface, and luminescence efficiency. Symbols: $\Psi^{(/)}=$dihedral angle between chromophoric units $\mathrm{A}^{(/)}$and $\mathrm{B}^{(/)}, \theta^{(/)}=$rotation amplitude, and $\omega^{(/)}=$frequency or wavenumber. be varied in a wider range (theoretically $|0|^{\circ}-|90|^{\circ}$ ). Much energy is dissipated by the large twisting motions, leading to a weaker PL of the luminogen in the solutions.

Intuitively, one would consider that adding more aromatic rings to an already emissive molecule should further extend its $\pi$-conjugation and make it more emissive. ${ }^{95}$ This may not always be true. For example, BODIPY $\mathbf{4 2}$ is an efficient luminophore. Its derivative with one more phenyl ring (43), however, is less emissive: the $\Phi_{\mathrm{F}}$ value of its solution is $c a$. 4.3-fold lower than that of its parent $\mathbf{4 2}$ (Fig. 18). ${ }^{96}$ This can be understood by the model discussed above. As the phenyl ring in 43 is not coplanar with the BODIPY plate $\left(\psi>0^{\circ}\right)$, the torsional motion of the phenyl rotor around the $\mathrm{C}-\mathrm{C}$ single bond is active $\left(\theta>0^{\circ}\right)$, which non-radiatively deactivates the excitons of $\mathbf{4 3}$ to a great extent and makes its solution less emissive. On the other hand, attaching two methyl groups to the 1 - and 7-positions of $\mathbf{4 3}$ poses severe steric hindrance to the twisting motion of the phenyl ring. ${ }^{97}$ As a result of the molecular RIR process, $\mathbf{4 4}$ becomes more emissive than $\mathbf{4 3}$, although the $\psi$ value of $\mathbf{4 4}$ is larger $\left(\sim 90^{\circ}\right)$.

According to the pictorial model presented in Fig. 17, luminescence behaviour of a molecule in a solution can be predicted from $\psi$ (or planarity) and $\theta$ (or rotatability), in which the structural rigidity plays a decisive role. To further prove the usefulness of the model, we computed the energy barriers to the torsional motions of the phenyl rings at the 8-positions of $\mathbf{4 3}$ and $\mathbf{4 4} .^{93}$ The calculated energy curves are shown in Fig. 18. Clearly, the phenyl group can rotate much more easily in $\mathbf{4 3}$ than in $\mathbf{4 4}$, owing to the much smaller rotation barriers in the former system. Notably, the phenyl ring in $\mathbf{4 4}$ can undergo twisting motions in the low $\theta$ region $\left(<30^{\circ}\right)$ as freely as $\mathbf{4 3}$ does. This explains why the $\Phi_{\mathrm{F}, \mathrm{s}}$ value of 44 is smaller than that of its parent form (42) carrying no rotatable phenyl ring.

A more dramatic change in the emission efficiency with increasing number of phenyl rings is observed in a pair of silole derivatives 45 and 46 (Chart 10). ${ }^{20,98}$ Luminophore 45 has two phenyl rings at the 2,5-positions that are relatively coplanar with the silole core $\left(\psi=7.8^{\circ}\right)$. This conformation allows some extent of $\pi$-electron delocalization between the phenyl and silolyl rings, which makes the structure of $\mathbf{4 5}$ relatively rigid and its solution relatively emissive $\left(\Phi_{\mathrm{F}, \mathrm{s}}=29 \%\right)$. The emission is completely quenched when two more phenyl rings are
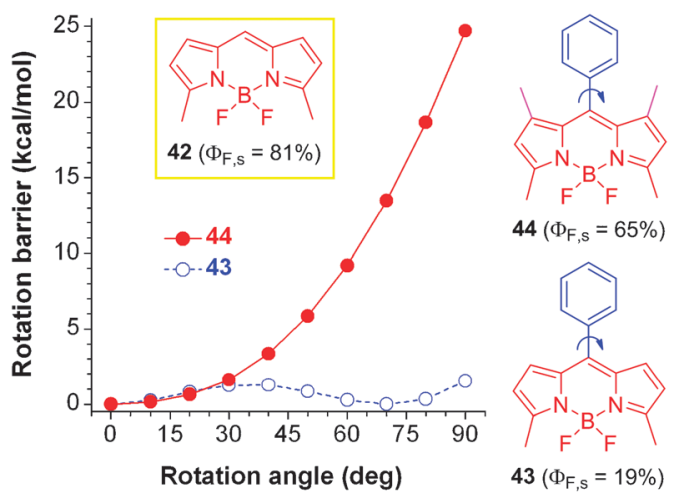

Fig. 18 Energy barriers as a function of rotational angels of phenyl rotors in $\mathbf{4 3}$ and $\mathbf{4 4}$, which are phenylated derivatives of $\mathbf{4 2}$. 
attached to the 3,4-positions of the silole core: the $\Phi_{\mathrm{F}, \mathrm{s}}$ value of the resultant luminogen $(\mathbf{4 6})$ is nearly nil $(0.063 \%)$. The phenyl rings of $\mathbf{4 6}$ at the 3,4-positions are themselves out of the plane of the silole core by around $57^{\circ}$ and in the meantime force the other phenyl rings at the 2,5-positions to take a more twisted conformation $\left(\psi \sim 44^{\circ}\right)$. The deviation from planarity and the escalation in rotatability of the phenyl rings work collectively to effectively quench the solution emission of $\mathbf{4 6}$.

\subsection{Intramolecular restrictions}

The model shown in Fig. 17 suggests that one can tune the emission performance of a molecule through modulating its conformational stability. ${ }^{99-101}$ Internally, a covalent linkage can lock or stabilise molecular conformation, hinder intramolecular rotation and enhance emission intensity. The $\Phi_{\mathrm{F}, \mathrm{s}}$ value of biphenyl (47), for example, is increased by 4.4 fold when a methylene linkage is introduced to fasten the phenyl rings in fluorene (48; Chart 11). ${ }^{102}$ The methylene bridge makes 48 more planar in conformation and averts the intramolecular rotations. Similar effect (i.e., a 5-fold change in $\Phi_{\mathrm{F}, \mathrm{s}}$ ) is observed in the structural pair $\mathbf{4 9}$ and $\mathbf{5 0} .^{36}$ The $\Phi_{\mathrm{F}, \mathrm{s}}$ values of $\mathbf{4 9}$ and $\mathbf{5 0}$ are respectively lower than those of $\mathbf{4 7}$ and $\mathbf{4 8}$. Evidently, the decreases in the $\Phi_{\mathrm{F}, \mathrm{s}}$ values stem from the introduction of conformationally flexible 2,2-diphenylethenyl units to the molecular structures of $\mathbf{4 9}$ and $\mathbf{5 0}$.

Luminogen $\mathbf{5 1}$ is a structural mimic of the emitting unit in the green fluorescence protein (GFP) ${ }^{103,104}$ It exists in two conformations ( $Z$ and $E$ ), both being non-fluorescent in the solution state, with $\Phi_{\mathrm{F}}$ values as low as $\leq 0.05 \%$ (Chart 12 ). The negligible emissions are theoretically attributable to the active rotations of the $\mathrm{C}-\mathrm{C}$ single bond (i.e., intramolecular twisting) and/or $\mathrm{C}=\mathrm{C}$ double bond (i.e., cis-trans or $E-Z$ isomerization). Though the probability for the occurrence of the photoinduced isomerization remains unclear (vide infra), significant contribution from the rotations of the $\mathrm{C}-\mathrm{C}$ single bond is beyond doubt. ${ }^{105}$ The ring-closing reaction of $Z-51$ with $\mathrm{BF}_{2}$ yields 52. Its locked ring conformation hinders the intramolecular rotations, making $\mathbf{5 2}$ intrinsically fluorescent in the solution state $\left(\Phi_{\mathrm{F}, \mathrm{s}}=86 \%\right)$.

Like the covalent chemical bonds, non-covalent physical interactions such as charge-transfer complexation can also trigger molecular RIR processes. ${ }^{106}$ As can be seen from the crystal structure of 53b, its electron-poor pentafluorophenyl rings are located right above the electron-rich flanking benzene rings of its anthracene core at an inter-ring distance of $3.36 \AA$ (Fig. 19). The A-D interaction prevents the intra-molecular torsions of the phenyl rotor units. Thanks to the crystallizationinduced structure rigidification, the crystal of 53b shows a very high fluorescence efficiency $\left(\Phi_{\mathrm{F}, \mathrm{c}}=99 \% ; c f ., \Phi_{\mathrm{F}, \mathrm{f}}=81 \%\right.$ for its film). When the pentafluorophenyl rings are replaced by
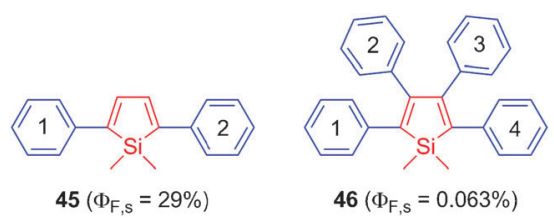

Chart 10 Effect of number of peripheral phenyl rotors on luminescence behaviour of silole luminogen.

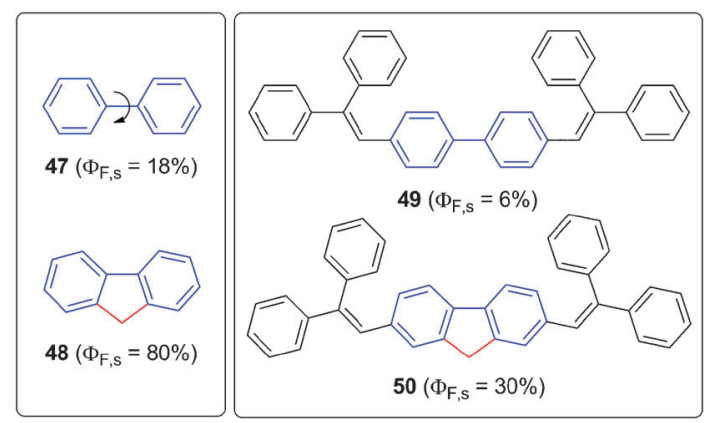

Chart 11 Examples illustrating RIR effect on luminescence behaviours of biphenyl-based luminogens.

pure phenyl rings, the fluorescence is weakened $\left(\Phi_{\mathrm{F}, \mathrm{c}}=75 \%\right.$ and $\Phi_{\mathrm{F}, \mathrm{f}}=43 \%$ for 53a). Without the D-A interaction, the phenyl units of 53a are located far from the anthracene stator. This permits some limited extent of torsional motions in the solid state, leading to the observed decrement in $\Phi_{\mathrm{F}}$.

\subsection{Intermolecular interactions}

Conformation of a molecule can be influenced by the forces not only within molecules (vide ut supra) but also between molecules. For example, molecular conformations have been found to be affected by the interplays with the surrounding environments: increasing viscosity, decreasing temperature and elevating pressure have resulted in enhancements in PL efficiencies, due to the externally imposed restraints to the structural variations. These examples have been discussed in our previous review articles ${ }^{29,75}$ and will not be elaborated here to avoid tautology. We will instead examine how other intermolecular processes and interactions perturb molecular conformations and influence luminescence behaviours.

In the example shown in Fig. 19, the structure of $\mathbf{5 3 b}$ was rigidified by an intramolecular complexation process in the crystalline state. The similar effect can be achieved through an intermolecular process. For example, the phenyl rings in TPE are twisted out of the central ethene plane by $\sim 50^{\circ} .{ }^{26}$ In the crystalline state, the propeller shape of the TPE molecule prevents $\pi-\pi$ stacking and excimer formation (Fig. 20A). On the other hand, multiple $\mathrm{C}-\mathrm{H} \cdots \pi$ hydrogen bonds are formed between the hydrogen atoms in the phenyl rings of one TPE molecule and the $\pi$ electrons of the phenyl rings of another adjacent TPE molecule (Fig. 20B). These hydrogen bonds stiffen the conformations of the TPE molecules and enhance their light emissions. ${ }^{107,108}$
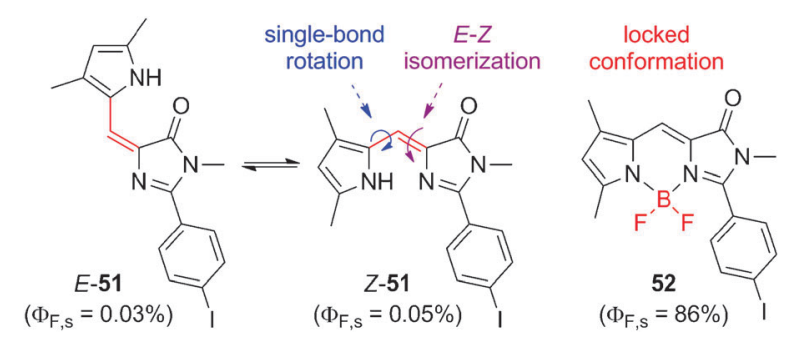

Chart 12 Effect of structural rigidification on emission behaviours of 4-(2-pyrrolyl)methylene-5-imidazolone derivatives. 

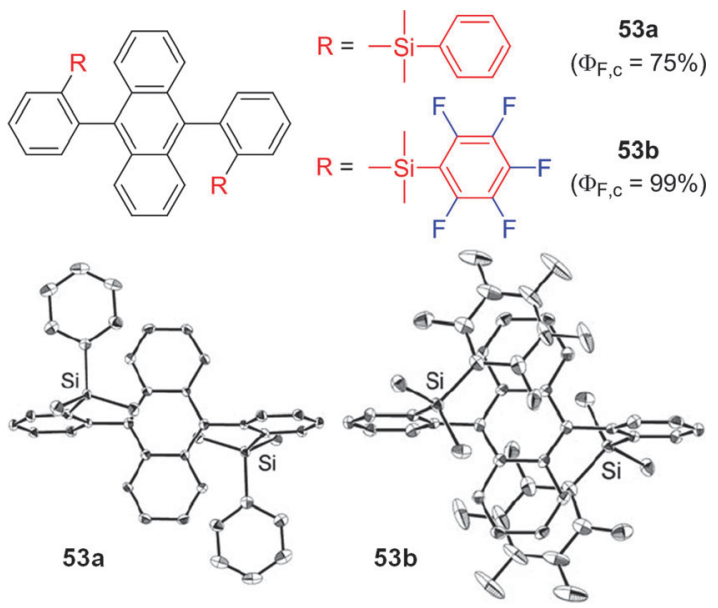

Fig. 19 RIR effect on luminescence efficiency of crystals of anthracenecored fluorophores (53). Reproduced with permission from ref. 106. Copyright (2009) The Royal Society of Chemistry.

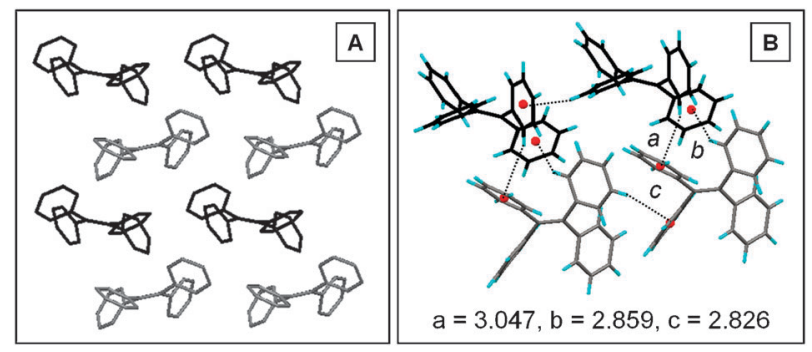

Fig. 20 (A) Molecular packing pattern of TPE in the crystalline state. (B) $\mathrm{C}-\mathrm{H} \cdots \pi$ hydrogen bonds with indicated distance $(\AA)$ between adjacent TPE molecules in the crystalline state. Reproduced with permission from ref. 26. Copyright (2007) American Institute of Physics.

The conformational rigidity of TPE is further established by the optical activity arisen from the atropisomerism of the crystals of its derivatives. It is known that achiral molecules can form chiral crystals if their conformational flexibility or orientational freedom is severely limited by a crystallization process. In the crystal structure of TPE, its four phenyl rings are twisted against the olefinic double bond and oriented in the same direction (Fig. 20), implying that its molecules can form atropisomeric crystals under appropriate conditions. To explore this possibility, we prepared a bisethynylated TPE derivative (54) and succeeded in growing crystals of its cis isomer. Strong Cotton effects are observed in the solid-state circular dichroism (CD) spectrum of the single crystals of cis-54 dispersed in a $\mathrm{KCl}$ matrix (Fig. 21), whereas its solutions in liquid media are completely CD inactive. Obviously, the observed enantiomorphism of cis-54 molecules is the result of a crystallization-triggered RIR process.

In principle, intramolecular rotations of any bulky groups, regardless of whether they are conjugated or non-conjugated, should consume energy and extinguish excited states. This proved the example shown in Fig. 22. A rhenium complex carrying two non-conjugated trimethylsilyl (TMS) rotors (55) was prepared by Panigati, Mercandelli and De Cola. ${ }^{109}$ The solutions of $\mathbf{5 5}$ are weakly luminescent but its crystals are highly emissive. The RIR process of the TMS rotors in the crystals was proposed to account for the AIE effect. The Re complex shows "colourful" emission behaviours: different forms of its crystals emit lights with different colours (green or orange; Fig. 22). The demonstration of the AIE effect in a luminogen carrying non-conjugated rotors is of importance, for it helps greatly to expand the scope of the structural design of AIE luminogens.

The RIR process was found to synchronise with the molecular recognition process in the biological systems. The emission of $\mathbf{5 6}$ in a denatured GFP is faint in a dilute solution (Fig. 23) ${ }^{103}$ In the native folding structure of GFP, 56 sits in the middle of an internal $\alpha$-helix directed along a rigid $\beta$-barrel axis. In the confined space of the barrel, 56 becomes highly emissive. The solution of stilbene derivative $\mathbf{5 7}$, like $\mathbf{5 6}$, is weakly fluorescent. Its emission is enhanced in the presence of an antibody due to the complex formation in the ligandbinding pocket of the rigid protein matrix. ${ }^{110,111}$ Obviously, the RIR mechanism has played a vital role in the emission processes.

Luminogen 57 can undergo two kinds of intramolecular rotations against the $\mathrm{C}-\mathrm{C}$ and $\mathrm{C}=\mathrm{C}$ bonds. Which or both of them have quenched the emission of $\mathbf{5 7}$ in the solution state? To answer this question, we synthesized TPE derivative 58, which has a $Z$-rich conformation $(93 \%)$ and whose $Z$-to- $E$ isomerization can be easily followed by NMR analysis (Fig. 24). Like its parent form TPE, 58 is AIE active. After its solution was irradiated by the xenon lamp in a fluorescence spectrometer for a prolonged period of time (14 $\mathrm{min}$ ), its molecular conformation reminded unchanged $(Z=93 \%$; Fig. 24C). After the solution was irradiated by a strong UV lamp, however, the $Z$-to- $E$ isomerization took place, producing a mixture consisting of an equal amount of $E$ and $Z$ isomers. The similar result was obtained in the system of stilbene: its $E-Z$ isomerization occurred only after its solution had been irradiated with a strong UV lamp.

The experimental data indicate that the single-bond intramolecular rotation takes place at all powers of the photoirradiations, whereas the double-bond $E-Z$ isomerization is power-dependent. Thus, when the solution of $\mathbf{5 8}$ is irradiated by the weak light from the xenon lamp in the fluorescence spectrometer, the photo-accelerated torsional motions of its
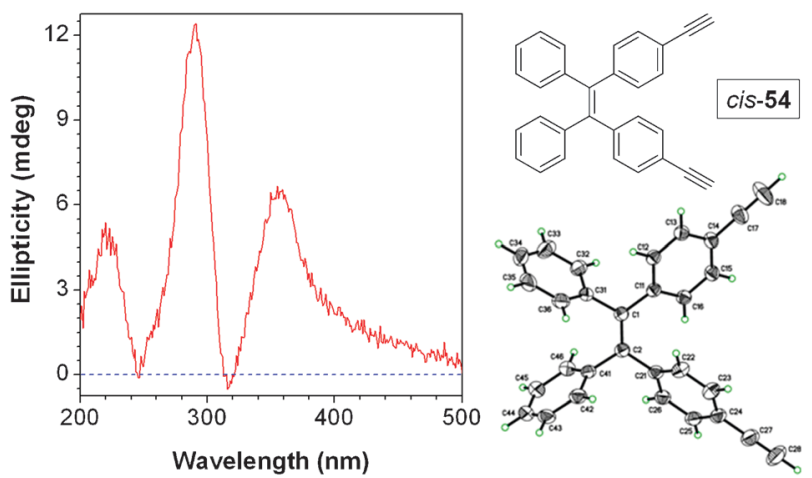

Fig. 21 Solid-state CD spectrum of microcrystals of cis-54 dispersed in $\mathrm{KCl}$ matrix. 

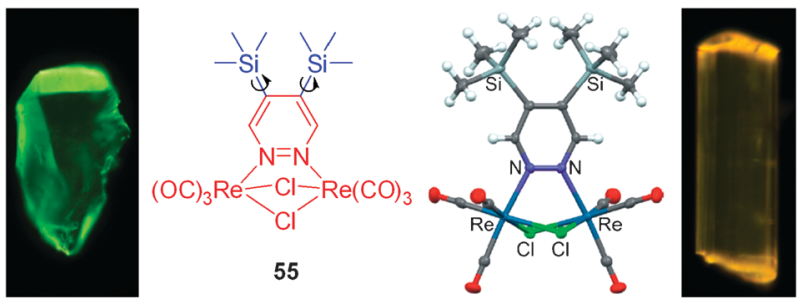

Fig. 22 Photographs of crystals of $\mathbf{5 5}$ taken under illumination of a UV lamp. Reproduced with permission from ref. 109. Copyright (2010) American Chemical Society.
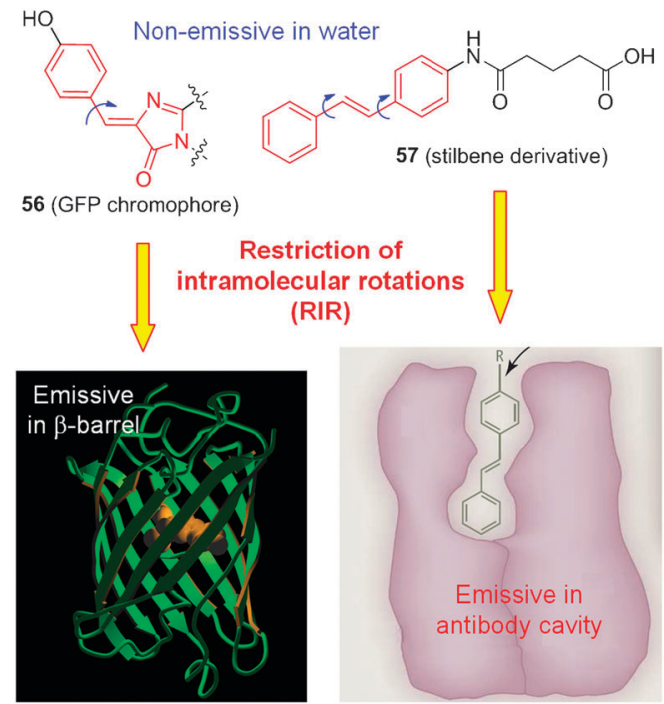

Fig. 23 RIR process turns on light emissions of GFP chromophore and antibody-stilbene complex. Reproduced with permission from ref. 103 [Copyright (2009) American Chemical Society] and 111 [Copyright (2008) American Association for the Advancement of Science].
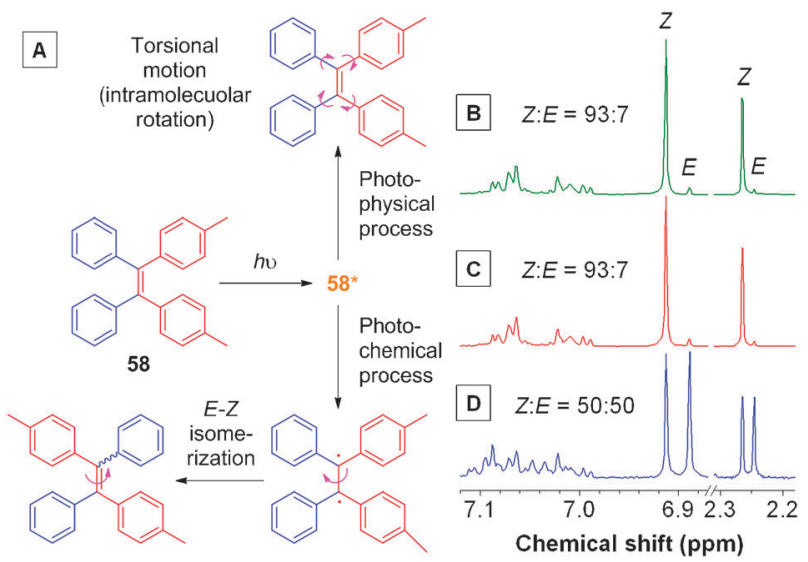

Fig. 24 (A) Possible pathways of photoinduced physical and chemical processes of $Z$-rich 1,2-diphenyl-1,2-(di- $p$-tolyl)ethene (58; $Z$ content $=$ $93 \%) .{ }^{1} \mathrm{H}$ NMR spectra of chloroform- $d$ solution of $\mathbf{5 8}$ (B) before and after (C) excitation with a xenon lamp in a PL spectrometer and (D) irradiation with a UV lamp $\left(\lambda_{\mathrm{ex}}=312 \mathrm{~nm}\right)$ for $14 \mathrm{~min}$.

phenyl rotors around the $\mathrm{C}-\mathrm{C}$ single bonds is the physical process that quenches its solution emission but brings about no change to its $Z / E$ content. The $E-Z$ isomerization requires higher energy because it involves a photochemical process that breaks down the $\pi$ bond in the $\mathrm{C}=\mathrm{C}$ double bond. The random rotations of the phenyl( $p$-tolyl)methyl units around the single bond resulted from the breakdown of the double bond lead to the formation of a mixture comprising of equal amounts $(50 \%)$ of the $E / Z$ isomers.

Under the normal settings of a common research laboratory, emission behaviour of a molecule is commonly studied by measuring its PL spectrum through exciting its solution with the xenon lamp in a spectrofluorometer. The spectral scan typically takes $<=1 \mathrm{~min}$ to finish and the $E-Z$ isomerization should not occur under such mild conditions. It can thus be concluded that it is the single-bond intramolecular rotation that has quenched the emission of an AIE luminogen in the solution state under normal laboratory conditions. The $E-Z$ isomerization, however, may happen when a luminogen is exposed to harsh irradiation by the strong laser beam from a confocal fluorescence microscope, causing undesired photobleaching.

\subsection{ACQ-to-AIE transformation}

As mentioned in the Introduction, traditional luminophores are commonly flat molecules with discotic shapes that can luminesce efficiently when they are genuinely dissolved or molecularly dispersed in their good solvents. In the solid or aggregate state, however, the luminophoric molecules can tightly pile up and thus experience strong $\pi-\pi$ stacking interactions. The undesirable effect of aggregate formation dominates in these systems, leading to the notorious ACQ effect. The ACQ problem must be properly tackled, because luminophores are often used as aggregate suspensions in the aqueous media or thin films in the solid state in their high-tech applications.

Various approaches have been taken to hamper aggregate formation. The attachment of alicyclics (e.g., cyclohexyl unit), wrapping by surfactants (e.g., sodium dodecylsulfate) and blending with polymers [e.g., poly(methyl methacrylate) or PMMA] are the widely employed methods for impeding chromophore aggregation. These approaches, however, are often followed by undesired side effects. The use of the nonconjugated alicyclics, surfactants and polymers, for example, dilutes fluorophore density and baffle charge transport in the electroluminescence (EL) devices. The traditional strategies for tackling the ACQ issue are hence far from ideal, because the ACQ effect is alleviated at the expense of other useful properties of the luminophores.

A win-win situation is the eradication of the ACQ effect without sacrificing the functional properties of the molecule. Based on our mechanistic understanding, it is envisaged that structural modifications of traditional luminophores by AIE groups may work as a new effective way to transform ACQ luminophores to AIE luminogens. ${ }^{112}$ As an AIE unit is itself electronically conjugated, it is expected that molecular meld of ACQ and AIE units would generate a luminogen that is ACQ-free and AIE-active, while keeping desired peculiarity of its building blocks or components.

Before elaborating our new strategy for the ACQ-to-AIE transformation, a new numerical parameter or an $\alpha$ factor for 
quantifying the change in the emission efficiency from the solution state to the aggregate state is defined as follows: ${ }^{113}$

$$
\alpha=\frac{\Phi_{\mathrm{F}, \mathrm{a}}}{\Phi_{\mathrm{F}, \mathrm{s}}}
$$

where $\Phi_{\mathrm{F}, \mathrm{a}}$ is the fluorescence quantum yield of aggregates. ( $\Phi_{\mathrm{F}, \mathrm{a}}$ in the equation can be substituted by $\Phi_{\mathrm{F}, \mathrm{f}}$ or $\Phi_{\mathrm{F}, \mathrm{c}}$, when the morphology of the aggregates is known to be an amorphous film or crystalline powder). Thus, by definition, the $\alpha$ value for a luminogen with AIE activity $\left(\alpha_{\mathrm{AIE}}\right)$ is $>1$ and a big $\alpha_{\text {AIE }}$ value means a profound AIE effect, whilst the $\alpha$ value for a luminophore with ACQ taint $\left(\alpha_{\mathrm{ACQ}}\right)$ is $<1$ and a small $\alpha_{\mathrm{ACQ}}$ value means a severe ACQ effect.

The flat molecule of anthracene (59) is ACQ active $\left(\alpha_{\mathrm{ACQ}}=\right.$ 0.5 ; Chart 13). It is transformed to an AIE luminogen by decorating it with a TPE unit. The solution of adduct $\mathbf{6 0}$ is non-emissive but its aggregates luminesce very efficiently $\left(\Phi_{\mathrm{F}, \mathrm{a}}=100 \%\right)$, giving an $\alpha_{\mathrm{AIE}}$ value as high as $357 .{ }^{113}$ This strategy works equally well for other ACQ molecules. ${ }^{114,115}$ Embellishing an ACQ-active TPA core with multiple AIE-active TPE peripherals, for example, affords adduct $\mathbf{6 2}$ with combined advantages of the two components: holetransport capability (TPA) and AIE activity (TPE). Thanks to the electronic conjugation of the TPE component, the excellent hole-transporting property of the TPA component is well retained in $\mathbf{6 2}$, experimentally verifying the rationale and feasibility of our new strategy.

Like anthracene, pyrene is also an ACQ luminophore. ${ }^{7,116}$ In the low concentration region $\left(c<10^{-5} \mathrm{M}\right)$, its emission is increased with an increase in concentration (Fig. 25A). The spectral peak is located at $c a .390 \mathrm{~nm}$, due to the emission of its monomeric species in the molecular solution. When its concentration is increased to $10^{-4} \mathrm{M}$, its emission becomes weaker. Further increasing the concentration leads to further decrease in its emission intensity. At $c=10^{-1} \mathrm{M}$, the peak at $\sim 390 \mathrm{~nm}$ disappears, while a small bump appears at $c a$. $477 \mathrm{~nm}$ in the redder spectral region, indicative of a change from monomer-to-excimer emission. Attaching a TPE unit to the pyrene structure creates adduct $\mathbf{6 3}$ (Fig. 26), which is totally free of concentration quenching effect. As can be seen

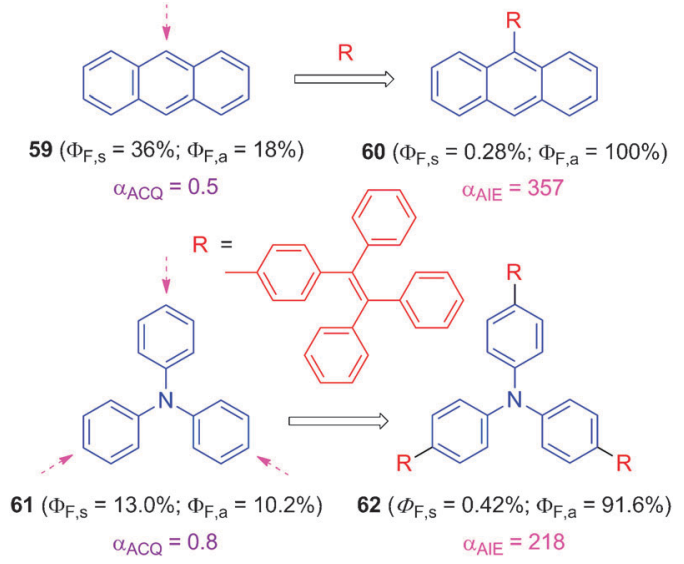

Chart 13 A new strategy for solving the ACQ problem: attachment of AIE groups to ACQ units results in the formation of new luminogens with high solid-state emission efficiencies.

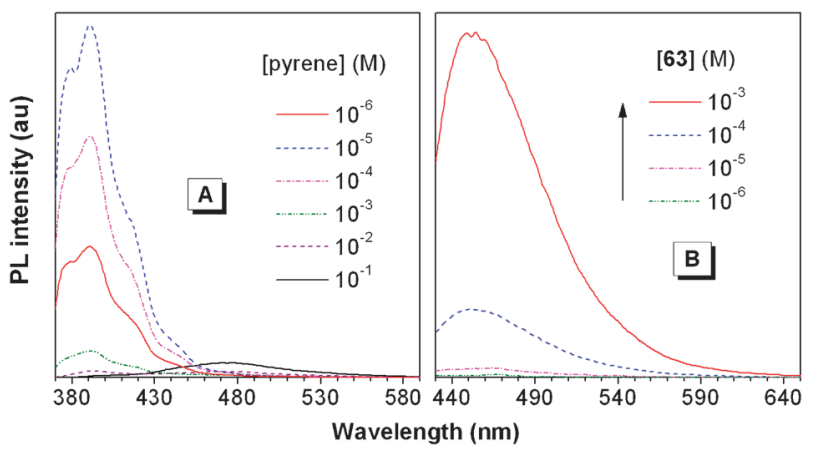

Fig. 25 Concentration effects on emission behaviours of THF solutions of (A) pyrene and (B) 63.

from Fig. 25B, its emission is monotonically intensified with an increase in concentration.

To gain more insight into the ACQ-to-AIE transformation process, we scrutinized the molecular structure and crystal packing of luminogen $63 .{ }^{117}$ The adduct assumes a highly twisted conformation: the torsion angle between the pyrene plate and the phenyl ring of TPE is as big as $66.7^{\circ}$ (Fig. 26). The molecules of $\mathbf{6 3}$ pack in an antiparallel fashion: a planar pyrene plate is inserted into two TPE units, while a TPE unit is sandwiched between two pyrene plates. This is a unique case of "site isolation": the separation of the pyrene plate by the propeller-shaped TPE units isolates the pyrene site and prevents the $\pi-\pi$ stacking of the pyrene units as well as their excimer formation. ${ }^{69}$

$\mathrm{C}-\mathrm{H} \cdots \pi$ hydrogen bonds are formed among the hydrogen atoms of a TPE unit in one molecule of adduct $\mathbf{6 3}$ and the $\pi$ electrons of the pyrene plate in an adjacent molecule, which further rigidifies the structure of $\mathbf{6 3}$ in the crystalline state. The packing motif in the crystal of $\mathbf{6 3}$ resembles an anchor: the TPE units are robustly docked on the pyrene decks with the aid of the multiple $\mathrm{C}-\mathrm{H} \cdots \pi$ bonds between the TPE and pyrene units. The molecular anchor structure strengthens the RIR process and boosts the radiative transition, endowing 63 with a solid-state emission efficiency of unity.

The conventional approaches mitigate the ACQ effect but bring about new problems. ${ }^{29}$ In contrast, our new approach solves the ACQ problem without causing undesired sideeffects. The examples discussed above show that embossing ACQ luminophores with TPE units is a general and versatile strategy for eliminating ACQ effect and generating new AIE luminogens. Our new strategy takes advantage of the natural process of aggregate formation and therefore does not suffer from temporal and spatial instabilities. The resultant adducts are strong emitters in the solid state, enabling them to find real-world technological applications, as to be discussed in the following section.

\section{Technological applications}

New concepts leads to new applications. In principle, the AIE effect can be utilized to do useful work wherever the RIR process is involved. The possibilities are therefore plentiful, limited probably only by our imagination. Many laboratories have worked on the exploration of high-tech applications of 


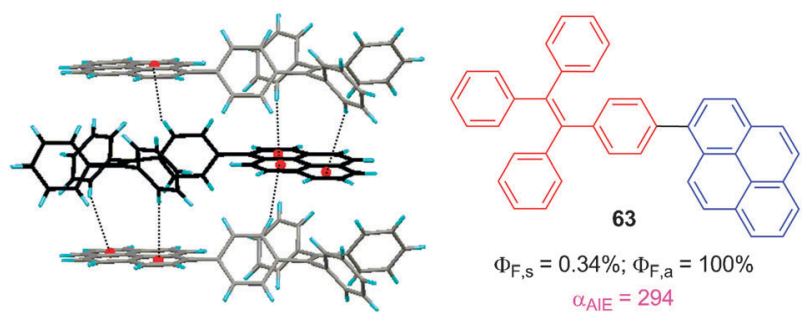

Fig. 26 Molecular anchor: docking of TPE units on pyrene decks via $\mathrm{C}-\mathrm{H} \cdots \mathrm{H}$ bonding (marked by dotted lines) endows crystals of $\mathbf{6 3}$ with emission efficiency of unity. Reproduced with permission from ref. 117. Copyright (2011) The Royal Society of Chemistry.

the AIE effect and much of the effort has been devoted to its utilities in optoelectronic and sensory systems. The device performances of the OLEDs based on the conventional flat luminophores have been unsatisfactory, mainly due to the ACQ problem. ${ }^{118}$ The AIE luminogens are emissive in the aggregate state and are therefore promising materials for the fabrication of efficient OLEDs. This proves to be true: an silole-based OLED shows an external quantum yield $\left(\eta_{\mathrm{EL}}\right)$ as high as $8 \%$, approaching the limit of the possible for an EL device based on an organic singlet emitter. ${ }^{119}$

Conventional fluorescent sensors commonly operate in a PL turn-off mode: an initially highly emissive dye becomes nonfluorescent when its molecules are induced to aggregate in the presence of a chemical species or biological analyte. The unusual AIE effect allows the development of new type of sensing system that functions in a PL turn-on mode by taking advantage of luminogen aggregation. Such a lighting-up sensor is superior to its fading-out counterpart: it is more sensitive and less likely to generate false-positive signals. ${ }^{120}$ To date, a large variety of AIE-based chemical sensors and biological probes have been successfully invented. ${ }^{121,122}$ In this section, we will discuss some recent progress in the area of technological applications of the AIE effect, with the emphasis on the work reported after the publication of our previous review in $2009 .^{29}$

\subsection{Electroluminescence devices}

To attain full-colour display, AIE luminogens with emission colours covering the whole range of visible lights have been designed and prepared. ${ }^{29,75,115,123-126}$ Some examples of the luminogens are shown in Chart 14. Many OLEDs using the AIE luminogens as the emitting layers have been fabricated. For example, an EL device has been constructed from 64, a newly developed AIE luminogen consisting of a pyrene core and four TPE peripheries. ${ }^{115}$ The configuration of the device is ITO/NPB/64/TPBi/LiF/Al, where ITO = indium tin oxide, $\mathrm{NPB}=N, N^{\prime}$-bis(1-naphthalenyl)- $N, N^{\prime}$-bis(phenyl)benzidine and $\mathrm{TPBi}=1,3,5$-tris(1-phenyl-1 $H$-benzo[ $d]$ imidazol-2-yl)benzene. The OLED is turned on at $3.6 \mathrm{~V}$ and emits a sky blue light of $488 \mathrm{~nm}$ with a maximum luminance $\left(L_{\max }\right)$ of $36300 \mathrm{~cd} \mathrm{~m}^{-2}$ (Table 1). Its current and power efficiencies are $12.3 \mathrm{~cd} \mathrm{~A}^{-1}$ and $7.0 \mathrm{~lm} \mathrm{~W} \mathrm{~W}^{-1}$, respectively. Its $\eta_{\mathrm{EL}}$ is very high $(5.0 \%)$, putting it into the list of the most efficient OLEDs fabricated so far from pure organic emitters.

TPA is well-known for its fast hole mobility and has been widely used as a building block for the construction of hole-transporting molecules. ${ }^{124}$ Attaching two TPA groups to the two sides of a TPE core results in the formation of an AIE luminogen (65) that shows not only high emission efficiency but also superb hole-transporting properties. ${ }^{125}$ In other words, 65 serves dual roles as that of light emitter and hole transporter, which allows simplification of device configuration without debilitating device performance. Thus without using an extra hole-transporting layer, an OLED based on $\mathbf{6 5}$ emits a green light of $512 \mathrm{~nm}$ in high luminescence $\left(L_{\max }=\right.$ $\left.33770 \mathrm{~cd} \mathrm{~m}^{-2}\right)$ and efficiency $\left(\eta_{\mathrm{EL}}=4.4 \%\right.$; Table 1$)$.

A red emitter is an indispensable component for an OLED unit in a full-colour display system. Traditional red emitters often contain big fused polynuclear cores, which are apt to aggregate and suffer from the ACQ problem. Red emitters with appreciable efficiencies in the solid state are rare and thus in great demand. ${ }^{45,49,126}$ With the help of the electronwithdrawing thiadiazole unit, the film of $\mathbf{6 6}$ emits in the red region $(610 \mathrm{~nm})$ with a $\Phi_{\mathrm{F}, \mathrm{f}}$ of $55.4 \%{ }^{125}$ Its OLED emits an orange-red light with $L_{\max }$ and $\eta_{\mathrm{EL}}$ values of $8333 \mathrm{~cd} \mathrm{~m}^{-2}$ and $3.0 \%$, respectively (Fig. 27), superior to those of the existing doped or non-doped orange-red OLEDs. A series of arylaminofumaronitrile-based AIE molecules with pure red light emissions have been developed by Chen et al., which have been found to show satisfactory OLED performances. ${ }^{126}$

A non-doped white OLED (WOLED) has been fabricated by using a blend of cyan and orange luminogens $(64 / 66)$ as the emitting layer. ${ }^{125}$ The WOLED emits a bright white light (Fig. 27), with a chromaticity coordinate of $(0.38,0.41)$. The performances of the WOLED are impressive: its $V_{\text {on }}, \eta_{\mathrm{C}}$ and $\eta_{\mathrm{P}}$ values are $6.0 \mathrm{~V}, 7.4 \mathrm{~cd} \mathrm{~A}^{-1}$ and $4.0 \mathrm{~lm} \mathrm{~W}^{-1}$, respectively. Performances of traditional doped-WOLEDs are sensitively affected by variations in fabrication conditions, such as film morphology, doping concentration, dopant distribution and layer thickness. On the contrary, the non-doped AIE-based WOLED is insensitive to fabrication conditions: it is easy to manufacture and thus suitable for mass production.

\subsection{Fluorescence sensors}

AIE luminogens are useful analytical tools and have been judiciously utilized as sensitive and selective chemosensors and bioprobes of turn-on type. ${ }^{28,29}$ Through proper structural designs, AIE molecules with charged and chelating groups have been prepared and used for detecting and quantifying trace amounts of environmental pollutants $\left(\mathrm{Hg}^{2+}, \mathrm{CN}^{-}\right){ }^{28,127}$ In many of these sensing systems, the free AIE molecules in the solutions are induced to form aggregates by the analytes via

Table 1 Performances of OLED devices based on AIE luminogens ${ }^{a}$

\begin{tabular}{lllrrrr}
\hline Emitter & $\lambda_{\max }$ & $V_{\text {on }}$ & \multicolumn{1}{c}{$\eta_{\mathrm{C}}$} & \multicolumn{1}{c}{$\eta_{\mathrm{P}}$} & \multicolumn{1}{c}{$L_{\max }$} & $\eta_{\mathrm{EL}}$ \\
\hline $\mathbf{6 4}$ & 488 & 3.6 & 12.3 & 7.0 & 36300 & 5.0 \\
$\mathbf{6 5}$ & 512 & 3.2 & 13.0 & 11.0 & 33770 & 4.4 \\
$\mathbf{6 6}$ & 592 & 5.4 & 6.3 & 2.9 & 8333 & 3.0
\end{tabular}

${ }^{a}$ For device configurations, see ref. 114 and 124. Abbreviations: $\lambda_{\max }=$ emission maximum $(\mathrm{nm}), V_{\mathrm{on}}=$ turn-on voltage $(\mathrm{V}), \eta_{\mathrm{C}}=$ maximum current efficiency $\left(\mathrm{cd} \mathrm{A}^{-1}\right), \eta_{\mathrm{P}}=$ maximum power efficiency $\left(\mathrm{lm} \mathrm{W}^{-1}\right), L_{\max }=$ maximum luminance $\left(\mathrm{cd} \mathrm{m}^{-2}\right)$, and $\eta_{\mathrm{EL}}=$ maximum external EL efficiency $(\%)$. 


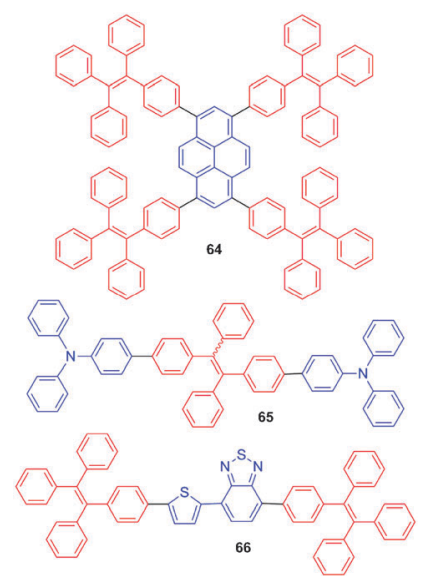

Chart 14 Examples of TPE derivatives showing high EL efficiencies.

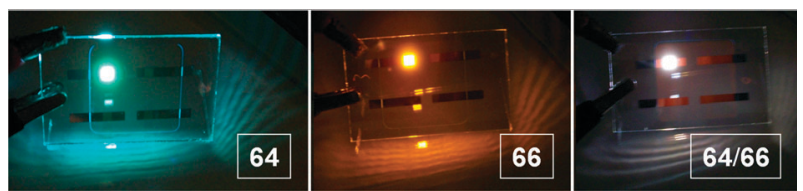

Fig. 27 Photographs of cyan, orange and white light-emitting EL devices using pure 64 and 66 and a mixture of them $(64 / 66)$ as emitting layers, respectively. Reproduced with permission from ref. 125. Copyright (2010) Bentham Science.

electrostatic attraction and coordinative complexation processes, which turn on the light emissions. ${ }^{128,129}$

Sensing of carbon-dioxide gas is of significant societal implications, because $\mathrm{CO}_{2}$ is a component of gas mixtures from many natural and anthropogenic processes with huge impacts on globe climate and human wellbeing (e.g., Fig. 28A). The current methods for $\mathrm{CO}_{2}$ sensing include electro-chemical (EC) and IR analyses. Both of these two methods have significant pitfalls. While the EC sensors require large amounts of power, the IR sensors pick up interfering $\mathrm{CO}$ signals in the gas mixtures. Our groups have developed an alternative scheme based on AIE luminogens for selective detection and quantitative assay of $\mathrm{CO}_{2}$ gas. ${ }^{130}$

In this scheme, HPS is dissolved in an amine solvent such as dipropylamine (DPA), giving a non-luminescent solution. When $\mathrm{CO}_{2}$ gas is bubbled through the solution, it reacts with DPA to yield a carbamate ionic liquid (CIL; Fig. 28B). The high viscosity and polarity of CIL slow down the torsional motions of the aryl rotors and induce the HPS molecules to aggregate. As the RIR process is set off, the HPS emission is turned on. With more $\mathrm{CO}_{2}$ gas bubbled through the solution, more CIL is formed. The PL intensity thus mirrors the $\mathrm{CO}_{2}$ level (Fig. 28C), which provides an inexpensive and visually discernable method for quantitative detection $\mathrm{CO}_{2}$ gas with applications as diverse as predicting volcanic eruptions and signalling danger in the environment. ${ }^{130}$

The threats from the increased uses of difficult-to-detect explosives in the terror attacks have become a global issue, which calls for urgent development of sensitive methods and processes for explosive detections. ${ }^{131}$ Many explosives are electron-deficient nitroaromatics, examples of which include 2,4,6-trinitrotoluene (TNT), 2,4-dinitrotoluene (DNT) and

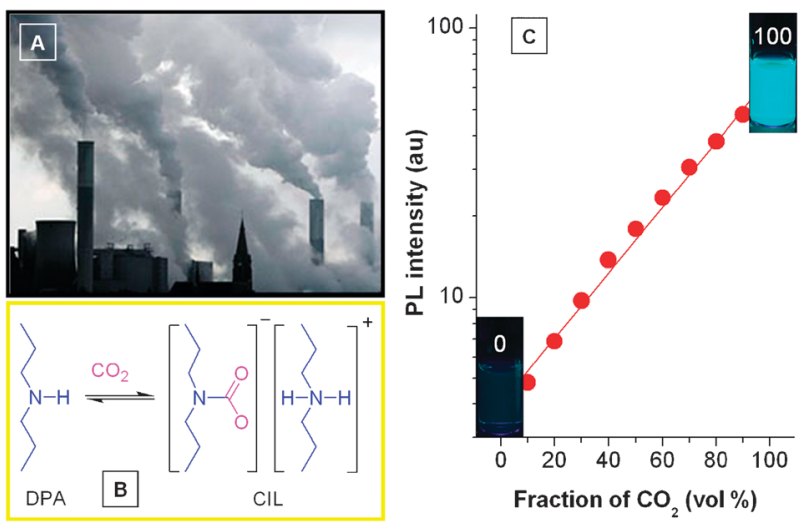

Fig. 28 (A) Photograph showing the discharge of $\mathrm{CO}_{2}$ gas from power plant into atmosphere. (B) Scheme showing the formation of carbamate ionic liquid (CIL) by the reaction of $\mathrm{CO}_{2}$ with dipropylamine (DPA). (C) Plot of emission intensity of HPS versus CIL fraction in the DPA/CIL mixture. Reproduced with permission from ref. 130. Copyright (2010) American Chemical Society.

2,4,6-trinitrophenol or picric acid (PA). On the other hand, the majority of AIE/AEE luminogens are electron-rich species. Their emissions are thus expected to be quenched by the explosives due to the charge-transfer interaction. The large surface areas of, and the numerous microscopic pores in, the AIE or AEE nanoaggregates may endow them with ultrahigh sensitivities. $^{132,133}$

An example of explosive detection system using an AEEactive hyperbranched polymer as the probe is shown in Fig. 29. The fluorescence of the nanoaggregates of 67 suspended in an aqueous mixture is progressively attenuated with the sequential addition of PA. ${ }^{134}$ The emission quenching can be clearly distinguished at a PA level ([PA]) as low as $1 \mathrm{ppm}$. The $I-[\mathrm{PA}]$ plot gives a swiftly upward-bending curve rather than a linear line, suggestive of a superamplification effect (Fig. 29C). The quenching constant is $1.35 \times$ $10^{5} \mathrm{~L} \mathrm{~mol}^{-1}$, much higher than those of other explosive sensors. The 3D topological structures of $\mathbf{6 7}$ and its aggregates provide many internal cavities for the explosive molecules and various diffusion paths for the excited states, leading to the extra-ordinary sensing performance. The superamplification effect has been observed in many AIE and AEE nanoaggregate systems, proving that it is a common feature for the AIE- and AEE-based sensing systems. ${ }^{76}$

Chiral recognition forms the mechanistic basis for HPLC enantioseparation, asymmetric catalysis, drug development, etc. An AIE chiral discrimination system was developed by Zheng et al., who prepared a pair of chiral carboxylic acids, D- and L-68, which emitted only when they were aggregated (Fig. 30). ${ }^{135}$ The L-form (L-68) enantioselectively formed nanoaggregates with $(1 R, 2 S)$-2-amino-1,2-diphenylethanol $[(1 R, 2 S)-69]$ but not its enantiomer $(1 S, 2 R)-69$. The nanoaggregates of $\mathbf{L - 6 8}-(1 R, 2 S)$-69 complex were very emissive, whereas the non-aggregative solution of the $\mathrm{L}-\mathbf{6 8} /(1 S, 2 R)-\mathbf{6 9}$ mixture was non-fluorescent. There was a linear relationship between the concentrations of $\mathrm{L}-\mathbf{6 8}$ and $(1 R, 2 S)-69$ in the aqueous medium, which enables quantitative analysis of the enantiomeric complexation process. 


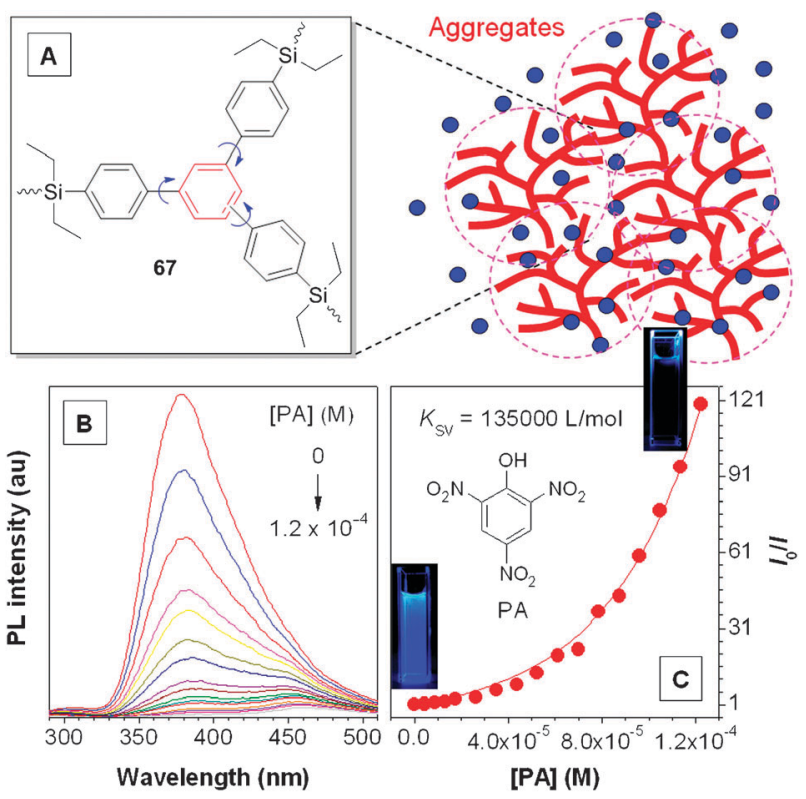

Fig. 29 (A) Schematic illustration of the explosive detection process by AEE-active hyperbranched polymer $\mathbf{6 7}$ in the aggregate state, where the red lines and blue dots represent the polymer branches and explosive molecules, respectively. (B) Change in the emission spectrum of nano-aggregates of $\mathbf{6 7}$ in a THF/water mixture $(1: 9 v / v)$ with the addition of different amounts of picric acid (PA). (C) Plot of emission intensity of $\mathbf{6 7}$ at $380 \mathrm{~nm}$ versus variation in PA concentration. Inset: Photographs of nanoaggregates of $\mathbf{6 7}$ in the absence and presence of PA taken under illumination of a UV lamp. Reproduced with permission from ref. 134. Copyright (2010) The Royal Society of Chemistry.

Through sensible structural designs, AIE luminogens can be tailored to serve different purposes. For example, a probe for thiol detection was developed by modifying TPE with a maleimide (MI) group. ${ }^{136}$ While TPE-MI adduct 70a is nonemissive in both solution and solid states due to the photoinduced electron transfer from TPE to MI unit, its thiolated derivative 70b shows AIE effect (Fig. 31A). In other words, 70a is a "caged" AIE luminogen. Its aggregates deposited on TLC plates can be used to differentiate cysteine from other amino acids through the thiol-ene click reaction (Fig. 31B). Because the click reaction is very fast and efficient at room temperature, 70a can also be used for labelling proteins

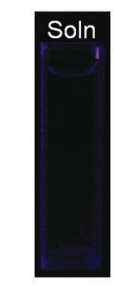

$L-68 /(1 S, 2 R)-69$ mixture

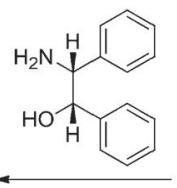

$(1 S, 2 R)-69$

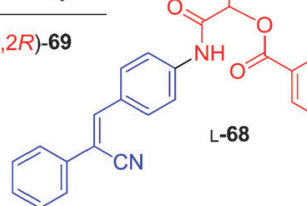

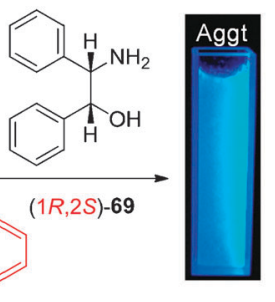
complex
L-68-(1R,2S)-69

Fig. 30 Fluorescent chiral recognition based on the aggregate formation induced by selective complexation of AIE-active luminogen L-68 with (1 $R, 2 S)$-2-amino-1,2-diphenylethanol (69). Reproduced with permission from ref. 135. Copyright (2009) American Chemical Society. carrying cysteine residues in the physiological media and in the poly(acrylamide) gel electrophoresis (PAGE) assays.

Carbohydrate-protein interactions have emerged as a hot research topic in recent decades because they are involved in many biological processes, such as cell adhesion, signal transduction and inflammation. ${ }^{137}$ The carbohydrate-protein interactions were utilized by Sanji et al. to developed new AIE biosensing systems. ${ }^{138} \mathrm{~A}$ phosphole oxide modified by sugar peripherals (71) was synthesized and used to detect lectins, proteins that bind with carbohydrates (Fig. 32). The nonemissive solution of $\mathbf{7 1}$ became emissive when Con A, a homotetramer lectin, was added to the buffer solution of $\mathbf{7 1}$. The $\alpha$-mannopyranoside moieties in $\mathbf{7 1}$ enabled its selective binding to Con $\mathrm{A}$, forming the emissive aggregates through multivalent sugar-lectin interactions.

Attachment of hydrophilic units such as hydroxyl, amino, ammonium and sulfonate groups to AIE luminogens can render them water soluble..$^{28,29}$ The adducts are non-emissive in the aqueous buffers but become emissive when bound to biomolecules. Guided by this design principle, a scheme for assaying adenosine- $5^{\prime}$-triphosphate (ATP) by an aminated silole (72) was established by Zhang et al. ${ }^{139}$ The cationic molecules of $\mathbf{7 2}$ form aggregates with the anionic molecules of ATP via electrostatic interactions (Fig. 33). The emission of $\mathbf{7 2}$ is increased with an increase in the ATP concentration, enabling quantitative ATP analysis. The emission of the 72-ATP ensemble is gradually weakened when it is incubated with phosphatase, an enzyme that catalyses ATP hydrolysis, suggesting that $\mathbf{7 2}$ can be used as a bioprobe for continuous in situ monitoring of the ATP hydrolysis process.

For a protein to perform its biological functions, it must assume a specific chain conformation. The development of luminescent tools for studying protein conformations is thus of obvious importance. ${ }^{129,140}$ Investigation of global stability of protein chains in the presence of denaturants has attracted much interest, as it offers mechanistic insights into protein (un)folding processes. Though intermediate states are often involved in the (un)folding processes of many proteins, they are difficult to detect due to the lack of appropriate probes.
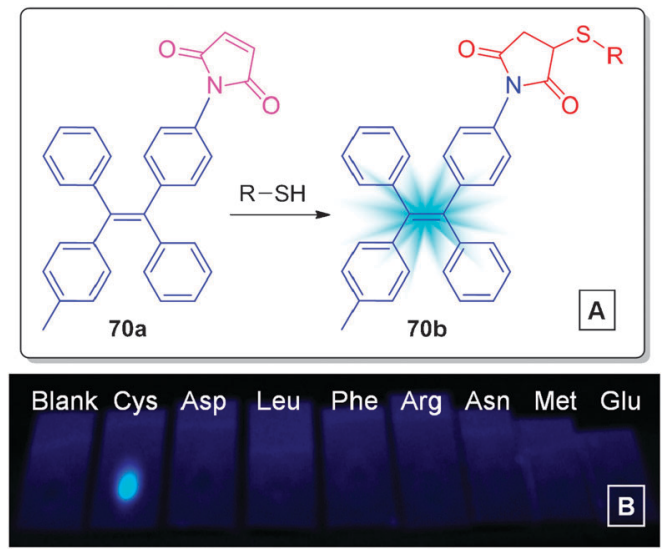

Fig. 31 (A) Fluorescence "turn-on" probe for specific thiol detection. (B) Discrimination of cysteine from other amino acids by thiol-ene click reaction of $\mathbf{7 0}$ on TLC plates. Photographs taken under illumination of a UV lamp. Reproduced with permission from ref. 136. Copyright (2010) Wiley-VCH. 


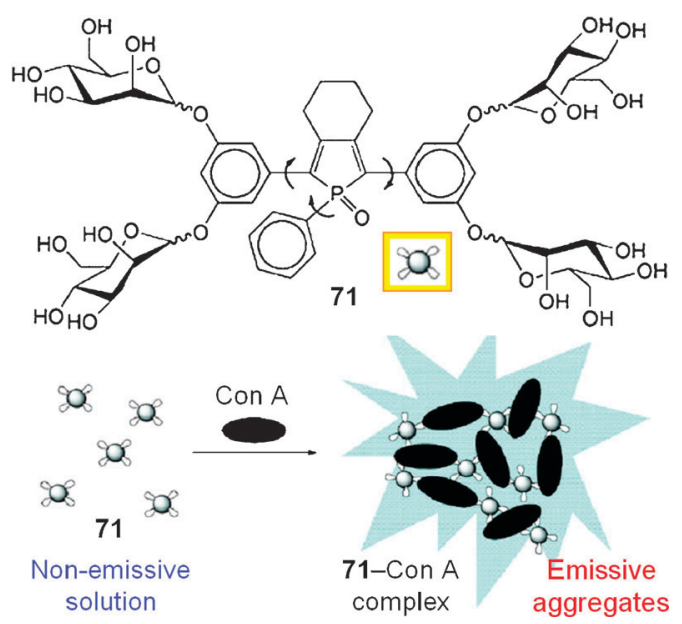

Fig. 32 Schematic representation of a fluorescence "turn-on" biosensor for Concanavalin A (Con A) detection by a sugar-containing phosphole (71). Reproduced with permission from ref. 138. Copyright (2009) American Chemical Society.

We developed an emission turn-on sensor for human serum albumin (HSA) using a sulfonated TPE (73) as probe. ${ }^{141}$ The emission of $\mathbf{7 3}$ in an aqueous buffer was switched on in the presence of HSA. The HSA-triggered emission allowed $\mathbf{7 3}$ to be used as a visualization agent in the PAGE assays.

HSA is a main protein component of the blood plasma. Its hydrophobic regions are capable of binding with insoluble endo- and exogenous compounds like fatty acids and drugs. The hydrophobicity of the phenyl rings of $\mathbf{7 3}$ prompted the luminogenic molecules to enter into and aggregate inside the hydrophobic cavities or pockets of the folding structures of HSA chains (Fig. 34). Utilizing the AIE nature of $\mathbf{7 3}$ and the Förster resonance energy transfer (FRET) from the intrinsic fluorescence of HSA at $350 \mathrm{~nm}$ to the AIE emission of $\mathbf{7 3}$ at $470 \mathrm{~nm}$, the unfolding trajectory of HSA chains induced by denaturant of guanidine hydrochloride $(\mathrm{GdnHCl})$ was traced, which revealed a three-step transition with a stable moltenglobule intermediate involved in the unfolding process. ${ }^{141}$

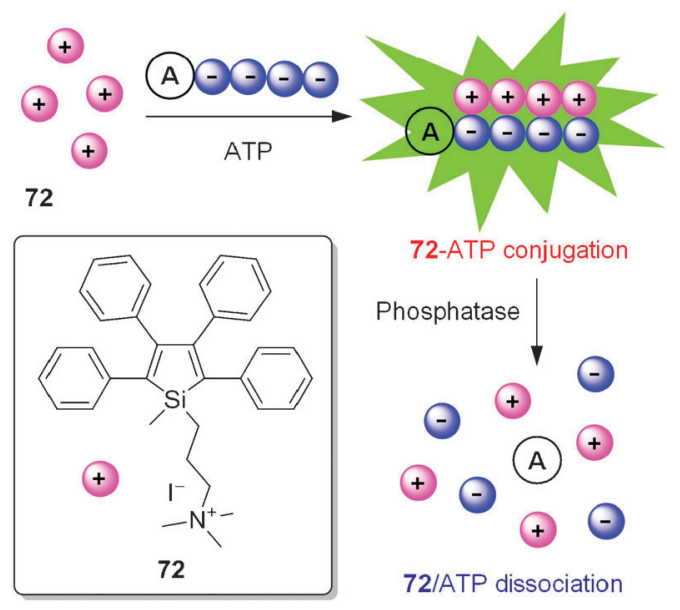

Fig. 33 ATP detection and phosphatase assay by ammoniated silole 72. Reproduced with permission from ref. 139. Copyright (2009) American Chemical Society.

\subsection{Cell imaging}

Because of their good water miscibility, ionic luminophores have often been used for cell imaging applications. However, at high concentrations, their charges can perturb membrane potential and cellular physiology. On the other hand, at low concentrations, the small numbers of the luminophores that have entered into the cellular interiors can be easily photobleached in the imaging process. During the cell division process and when the confluent cells are passaged, the intracellular dyes often diffuse back to the extracellular media owing to the existence of the concentration gradient. This leads to a decrease in the emission of the stained cells and a concurrent increase in the solution (or background) emission as well as a random staining in a cell co-culture system.

Aminated silole derivatives $\mathbf{7 4}$ are non-ionic luminogens that spontaneously aggregate into nanoparticles in aqueous media (Fig. 35A). The nanoparticles are cytocompatible and can be internalized via an endocytosis process. Due to their AIE nature and electrical neutrality, the luminogens can be used in high concentrations in cell imaging processes. The nanoaggregates indelibly stain the HeLa cells because the internalized particles are difficult to escape from the cellular compartments. The strong retention of the nanoparticles in the HeLa cells prevents the cross-staining between different cell lines and enables the fluorescence cell differentiation (Fig. 35B). The internalized particles are withheld inside the HeLa cells so robustly that they remain visible for a long period (up to four passages), permitting visual monitoring of the growth of a specific type of cell line (Fig. 35C).

As mentioned above, fluorophoric molecules suffer from the photobleaching problem when they are exposed to harsh laser light in the imaging systems. ${ }^{142}$ Silica nanoparticles are cytocompatible and optically transparent but fluorescently inactive. These attributes make them ideal host materials for the synthesis of fluorescent silica nanoparticles (FSNPs) for cell imaging applications. The silica matrix can serve as a protective shield, reducing the likelihood of the penetrations of oxygenic and other reactive species that may accelerate the photobleaching processes of fluorophores.

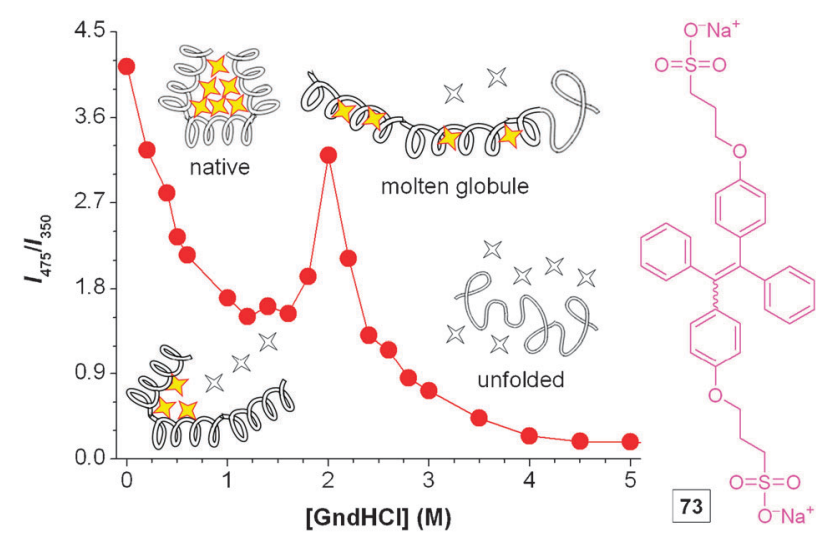

Fig. 34 Dependence of FRET ratio $\left(I_{470} / I_{350}\right)$ between HSA and 73 on the concentration of $\mathrm{GdnHCl}$ and illustration of fluorescence tracking of the GndHCl-induced HSA unfolding process. Reproduced with permission from ref. 141. Copyright (2010) American Chemical Society. 

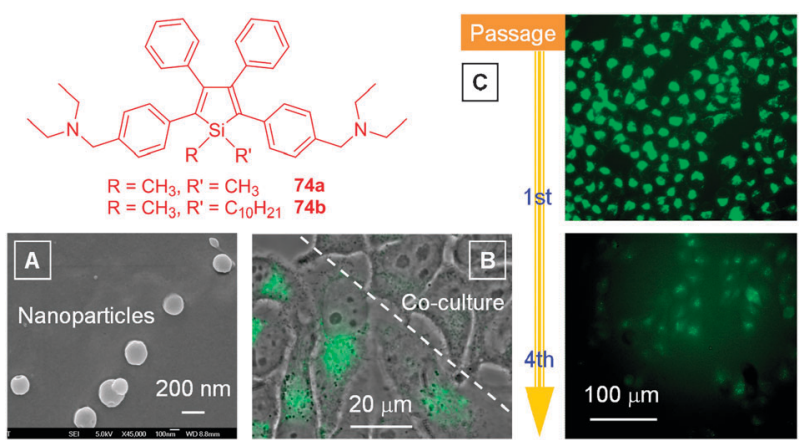

Fig. 35 (A) SEM image of nanoparticles of 74a formed in a THF/ water mixture with $70 \%$ water content. (B) Phase contrast and fluorescence images of the $\mathbf{7 4 b}$-stained HeLa cells co-cultured with unstained HEK 293T cells. (C) Fluorescence images of living HeLa cells stained with $5 \mu \mathrm{M}$ of $\mathbf{7 4 b}$ at the first and fourth passages.

FSNPs have been prepared by using such fluorophores as fluorescein isothiocyanate (FITC). However, high loading of FITC elicits ACQ problem, while low loading affords only weak emission. AIE luminogens become stronger emitters with increasing concentrations and are thus well suitable for FSNP fabrication. Using a triethoxysilylated TPE derivative (75) as a starting material, emissive FSNPs with a core-shell structure were prepared by a one-pot, surfactant-free, sol-gel process, as shown in Fig. 36. ${ }^{143}$ The FSNPs are uniformly sized, surface-charged and colloidally stable. Their sizes are tuneable in the range of $\sim 45-295 \mathrm{~nm}$ by varying the reaction conditions. The FSNPs emit a strong blue light, due to the AIE effect of their TPE components (Fig. 35B). The FSNPs pose no toxicity to living cells and can be used to selectively stain the cytoplasms of HeLa cells. ${ }^{143}$

Development of multifunctional nanomaterials is of great interest, for such materials are expected to serve as versatile legos for the constructions of advanced nanodevices. ${ }^{75,125,144}$ Meld of magnetic and fluorescent components via sol-gel procedures is envisaged to generate nanomaterials with dual functionalities, e.g., magnetic and fluorescent silica nanoparticles (MFSNPs), which may find biotech applications in fluorescent cell imaging and PAGE visualization as well as magnetic cell separation and tumour therapy. Fabrication of MFSNPs with strong fluorescence and high magnetisability, however, has been challenging, owing to the incompatibility between magnetic and fluorescent units: light emission is often quenched when inorganic magnets are hybridised with organic fluorophores.

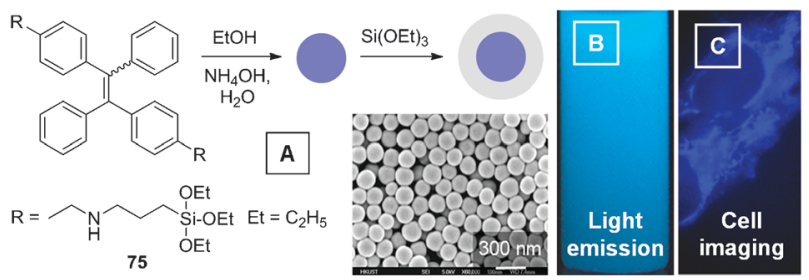

Fig. 36 (A) Fabrication, (B) light emission and (C) cell staining of core-shell fluorescent silica nanoparticles (FSNPs) consisting of fluorogenic cores and silica shells. Reproduced with permission from ref. 143. Copyright (2010) Wiley-VCH.
In the AIE systems, the luminescence is not from isolated molecules but assembled aggregates, which should be more robust than that from individual molecules of the traditional luminophores and thus more resistant to magnet quenching. Indeed, the nanoparticles consisting of magnetite cores and silolylsilica shells are highly emissive. ${ }^{144}$ The MFSNPs are uniform in diameter $(\sim 50 \mathrm{~nm})$, have a magnetisability of $\sim 6$ emu $\mathrm{g}^{-1}$ and can be readily modulated by a magnet bar (Fig. 37). ${ }^{75}$ They are readily internalized by HeLa cells, enabling cytoplasmic staining (Fig. 37C). Structural modifications of their surfaces by target-specific ligands such as folates and antibodies will yield nanoparticles that may be used for in vivo imaging of specific cells by MRI and for intracellular hyperthermia treatment based on magnetocaloric effect.

\subsection{Microstructured aggregates}

We are interested in morphosyntheses of organic nanowires, which are advantageous over their inorganic counterparts. Examples of the advantages include variety and flexibility in molecular selection, low cost fabrication via self-assembly, high-volume manufacture, and ready structure and property manipulations. ${ }^{58}$ In 2008, we prepared TPE-doped PMMA nanowires, which were a couple of hundred nanometres in diameter and several tens of micrometres in length. ${ }^{145}$ The individual microwires lased intensely at $372 \mathrm{~nm}$ with a full width at half maximum (FWHM) of $0.3 \mathrm{~nm}$ at $\sim 10 \mathrm{~K}$, which are respectively the shortest wavelength and the narrowest FWHM reported so far for a pure organic nanowire laser.

Park et al. used AIE luminogens to fabricate nanowires, whose emission colours could be blue, green, yellow or red, readily tuneable by varying the luminogen structures. ${ }^{146} \mathrm{We}$ achieved multicolour emissions from the same microfibres by changing excitation wavelength but not luminogen structure (Fig. 38). ${ }^{147}$ The microfibers were prepared by self-assembly of the molecules of 76, which emitted green, yellow and red lights when they were excited by UV, blue and green lights, respectively. This implies that the microfibres contain fibrils with different extents of $\pi$-conjugations, due to the assembly of the AIE molecules with different conformation structures, intermolecular interactions and packing arrangements.
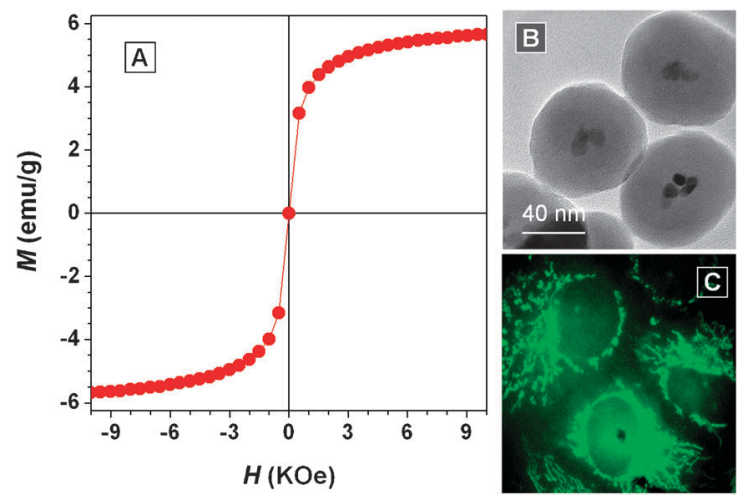

Fig. 37 (A) Plot of magnetisation $(M)$ versus applied magnetic field $(H)$ for silole-containing magnetic fluorescent nanoparticles at $300 \mathrm{~K}$. (B) TEM images of the nanoparticles and (C) fluorescence images of the HeLa cells stained by the nanoparticles. Reproduced with permission from ref. 144. Copyright (2011) Wiley-VCH. 


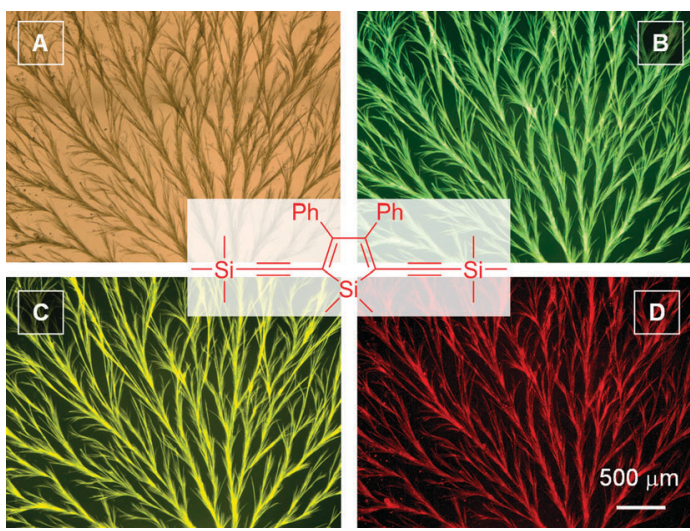

Fig. 38 (A) Photograph and (B-D) fluorescence images of microfibres of silole $\mathbf{7 6}$ taken (A) under normal lighting and upon excitations by (B) UV, (C) blue and (D) green lights. Reproduced with permission from ref. 147. Copyright (2009) Wiley-VCH.

The multicolour luminescence can be realised in not only ensembles of small molecules but also aggregates of large polymers. According to the reaction mechanism of the diyne polycyclotrimerization, hyperbranched polysilole $\mathbf{3 3}$ should contain unreacted ethynyl triple bonds, with most of them located in the periphery of the polymer sphere. ${ }^{148}$ Since the acetylenic units can be thermo- and photopolymerised, $\mathbf{3 3}$ should be easily cross-linked by heating and irradiation. The curing reaction is expected to extend to $\pi$-conjugation of the polymer. Indeed, the luminescence spectrum of the curing product (x-33) is red-shifted from that of its precursor (33) when excited by a UV light $(373 \mathrm{~nm}) .^{77}$

When the excitation wavelength is stepwise changed from $420 \mathrm{~nm}$ to $560 \mathrm{~nm}$ at an interval of $20 \mathrm{~nm}$, the emission peak of $\mathrm{x}-\mathbf{3 3}$ is progressively red-shifted from $610 \mathrm{~nm}$ to $735 \mathrm{~nm}$ with a monotonous decrease in the emission intensity (Fig. 39A). Under blue and green excitations, $x-33$ emits bright yellow (Fig. 39B) and red (Fig. 39C) lights, respectively, in agreement with the spectral data shown in Fig. 39A. The spectral and imaging data imply that $\mathrm{x}-\mathbf{3 3}$ contains segments with different $\pi$-conjugation lengths in different populations and that energy transfer channels are blocked in the cured product. This allows its segments to emit in response to their specifically matched stimuli, thus leading to the excitation wavelength-tuneable multicolour emissions.

We have been working on the development of advanced functional materials with both photonic and optical activities, particularly luminescent and mesomorphic properties. ${ }^{149,150}$ Mesogenic molecules are commonly rod-like or disc-shaped. This structural feature facilitates organizational packing of mesogens but incurs ACQ problem of luminophores. ${ }^{151}$ We have recently fused AIE luminogens and calamitic mesogens at molecular level to examine whether this would lead to the generation of light-emitting and liquid-crystalline molecules. In our structural design, TPE and 1-cyclohexyl-4(phenyl-ethynyl)benzene are employed as luminogen and mesogen, respectively. Two examples of such molecules are shown in Fig. 40, where single and multiple calamitic mesogens are attached to a TPE unit to yield $\mathbf{7 7}$ and $\mathbf{7 8}$, respectively.

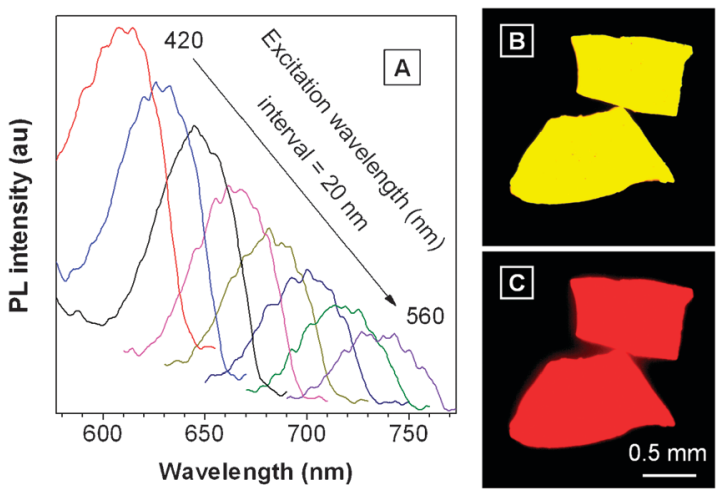

Fig. 39 (A) Excitation wavelength-dependent emission spectra of the powdery sample of $\mathbf{3 3}$ after thermal curing. Fluorescence images of the thermally cured powder of $\mathbf{3 3}$ taken under illumination of (B) blue and (C) green lights. Reproduced with permission from ref. 77. Copyright (2010) American Chemical Society.

Both of the two molecules were found to exhibit liquid crystallinity, indicating that the incorporation of the TPE moiety has not wrecked the organizational packing of the mesogens. Thermal analysis revealed that 77 underwent a mesophase transition at $\sim 70{ }^{\circ} \mathrm{C}$. Mesomorphic textures were observed under polarized optical microscope at temperatures higher than $\mathrm{ca} .70{ }^{\circ} \mathrm{C}$. The texture taken at $145^{\circ} \mathrm{C}$ is shown in Fig. $40 \mathrm{~A}$ as an example: the optical anisotropy of the texture unmistakably proves the liquid crystallinity of 77 . Similarly, mesomorphic textures were observed for $\mathbf{7 8}$ in its liquidcrystalline temperature region: for example, it displayed a typical focal conic texture at $190{ }^{\circ} \mathrm{C}$ (Fig. 40B).

The AIE characteristics are well preserved in these liquid crystalline systems. The $\Phi_{\mathrm{F}, \mathrm{s}}$ and $\Phi_{\mathrm{F}, \mathrm{f}}$ values for the solution and film of 77 are $0.68 \%$ and $55.4 \%$, respectively, giving an $\alpha_{\mathrm{AIE}}$ value as high as 81.5 . The PL spectrum of the film is centred at $\sim 480 \mathrm{~nm}$, which is analogous to the TPE spectrum, verifying that the TPE emission in $\mathbf{7 7}$ has not been quenched by the mesomorphic packing. The emission from the film of $\mathbf{7 8}$ is similarly efficient, with a $\Phi_{\mathrm{F}, \mathrm{f}}$ value as high as $67.4 \%$. The mesogenic and luminogenic properties of the molecules may
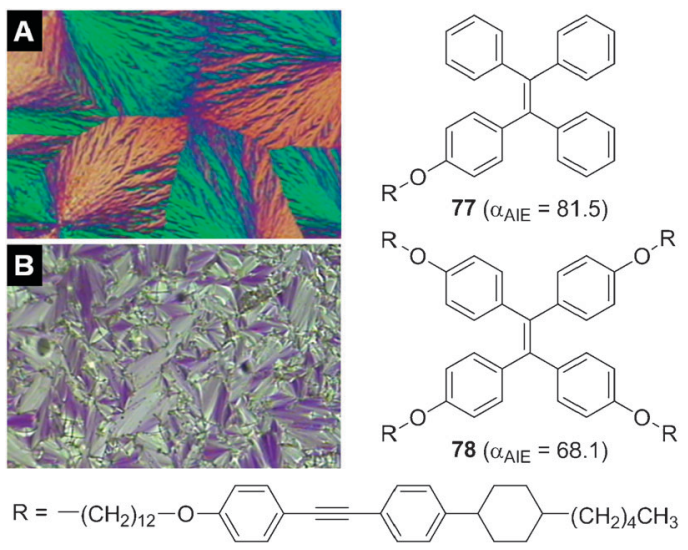

Fig. 40 Mesomorphic textures of luminogenic and mesogenic molecules (A) 77 and (B) 78; the photographs were taken under polarized optical microscope upon cooling the isotropic melts of the AIE-active liquid crystals to (A) $145^{\circ} \mathrm{C}$ and (B) $190{ }^{\circ} \mathrm{C}$. 
enable them to find novel applications as functional materials. For example, judicious utilizations of the light-emitting and liquid-crystalline materials may lead to the invention of new optical display systems with simple device structure and wide viewing angle, while circumventing the use of power-hungry backlight.

Development of intelligent materials capable of reversibly switching between different states is of great technological implications. Silole derivative $\mathbf{7 9}$ is such a smart material, which shows unique mechano- and thermochromisms. ${ }^{152,153}$ Thus, upon grinding, its emission colour is changed from yellow (79Y) to red (79R), while after heating at $150{ }^{\circ} \mathrm{C}$ for $30 \mathrm{~s}$, the red powder is restored to the yellow form (Fig. 41). $\mathrm{X}$-ray diffraction study reveals that the mechanochromism is associated with a mechanically induced morphology change from crystalline to amorphous state, in agreement with our early observation that amorphous form of an AIE luminogen often emits redder light than its crystalline counterpart. The colour change can be repeated many times by the grindingheating cycle, manifesting the excellent reversibility of the colour-switching process (Fig. 41B).

Park et al. synthesized stimuli-responsive gelators, whose morphologies and properties were reversibly and repeatedly tuneable by temperature, irradiation, $\mathrm{pH}$, counterion, etc. ${ }^{154}$ For example, the gel of $\mathbf{8 0 a}$ is fluorescent but its sol is nonemissive (Fig. 42). Thermo- and photoinduced protonations of its pyridine ring yield salt $\mathbf{8 0 b}$. The presence of the bulky sulfonate counterion disassembles the organogel, following which the fluorescence disappears (Fig. 42B). The gel-to-sol transition is reversible and the emission can be recovered by deprotonation of $\mathbf{8 0 b}$. Whilst long and entangled nanofibres are observed in the xerogel of $\mathbf{8 0 a}$, no such structures are found in the sol of $\mathbf{8 0 b}$ (Fig. 42C), substantiating the AIE nature of the luminogen.

Sensitive monitoring of thermally induced conformation change is of importance to the development of bioprobes, as biological processes and events are often accompanied with variations in body temperature. Biopolymers undergo conformational transitions at temperatures higher than the normothermia, leading to protein aggregation (fibrosis) with sometimes fatal consequences (e.g., brain death). $\operatorname{Poly}(N-$ isopropylacrylamide) (PNIPAM) is the best-known thermoresponsive synthetic polymer, whose conformation changes
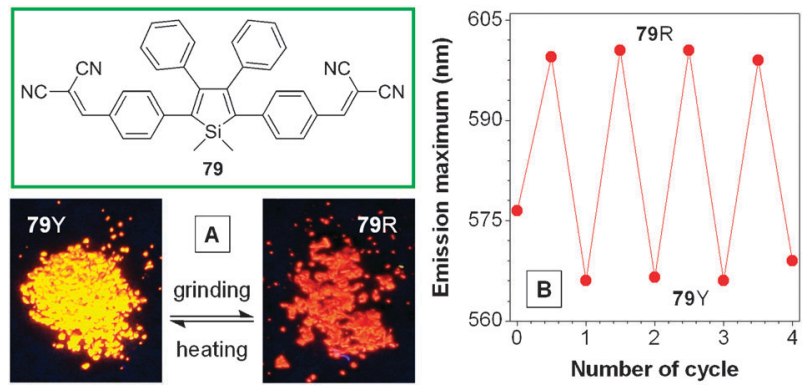

Fig. 41 Mechano- and thermochromisms of silole luminogen 79. (A) Photographs of its crystalline and amorphous powders taken under the illumination of a UV lamp and (B) plot showing reversible switching of its emission colour [yellow (Y) $\leftrightarrow$ red $(\mathrm{R})$ ] by grindingheating cycle.

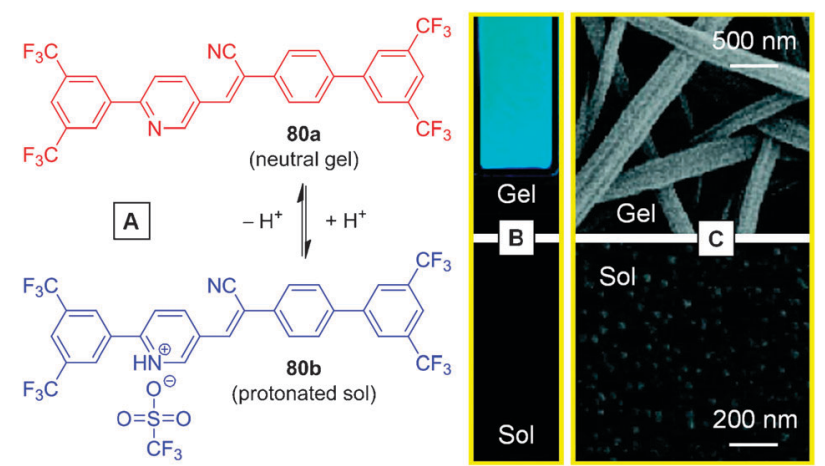

Fig. 42 (A) Reversible phase transition between neutral gel and ionized sol of 80. (B) Fluorescence and (C) SEM images of the gel and sol. Reproduced with permission from ref. 154. Copyright (2008) American Chemical Society.

from hydrated coil to dehydrated globule at a temperature close to the euthermia. PNIPAM is thus a nice synthetic model for the study of thermally induced conformational transitions in the natural polymer systems.

Labelling PNIPAM chain with TPE unit makes it possible to follow the conformational change of the polymer chain by the sensitive fluorescence technique. The temperature effect on the PL behaviour of the TPE-labelled PNIPAM (81) is shown in Fig. 43. At room temperature, the aqueous solution of $\mathbf{8 1}$ is weakly fluorescent. ${ }^{155}$ Little change in the emission intensity is recorded when the solution is warmed to $25^{\circ} \mathrm{C}$, while a small bump is observed in the temperature region of $25-29^{\circ} \mathrm{C}$. At $>29^{\circ} \mathrm{C}$, the PL is swiftly intensified with increasing temperature and reaches a maximum at $34.2^{\circ} \mathrm{C}$. Further heating leads to a continuing decrease in the light emission. When the aqueous mixture of $\mathbf{8 1}$ is cooled from $50{ }^{\circ} \mathrm{C}$ to $18{ }^{\circ} \mathrm{C}$, a largely reversible intensity-temperature curve is obtained, with the big peak and small bump reproducibly recorded in the similar temperature regions.

These experimental data offer the following mechanistic picture. When the solution is warmed to $25^{\circ} \mathrm{C}$, the PNIPAM chains commence to dehydrate and undergo the coil-globule transition. The tiny volume change is sensitively captured by the TPE probes: the volume shrinkage partially impedes their intramolecular motions and makes them somewhat emissive. In the temperature region of $29-34{ }^{\circ} \mathrm{C}$, the coil-to-globule
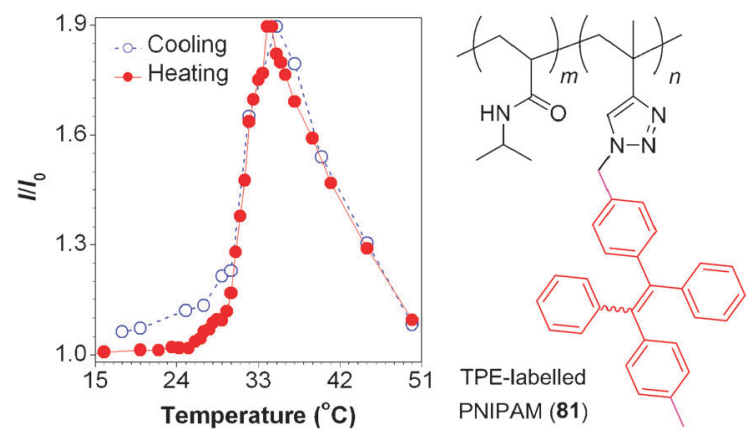

Fig. 43 Effect of temperature on emission intensity $(I)$ of TPE-labelled PNIPAM (81) in water; $I_{0}=$ intensity at room temperature. Reproduced with permission from ref. 155. Copyright (2009) The Royal Society of Chemistry. 


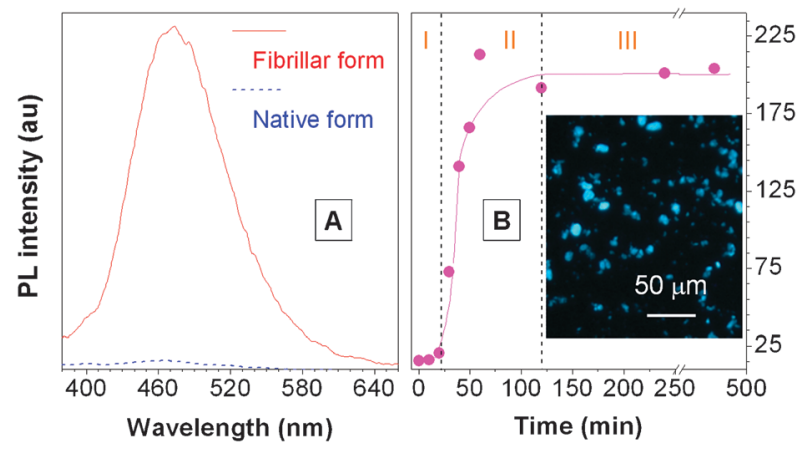

Fig. 44 (A) Emission spectra of 73 in the presence of native and fibrillar forms of bovine insulin. (B) Process of insulin fibrosis monitored by the change in the luminescence of 73. Inset: Fluorescence image of the insulin fibrils stained by $\mathbf{7 3}$.

transition becomes active. The large extent of dehydration results in compact polymer aggregates, which activates the RIR processes of the TPE labels and boosts their emissions. Further heating to $>34{ }^{\circ} \mathrm{C}$ may cause little change in the aggregate compactness but invigorate the molecular motions of the TPE labels, thus weakening their emissions. Clearly, the TPE labels function as non-monotonic probes capable of sensitively capturing detailed information about the thermal transitions of PNIPAM chain, which has not been accessible by other analytical techniques. ${ }^{155}$

Amyloids are insoluble fibrous aggregates of proteins, an excess accumulation of which in organs and tissues can lead to biological dysfunctions and pathological symptoms. An abnormal deposit of amyloids in brain, for example, causes neurodegenerative problems (e.g., Alzheimer, Parkinson and Huntington's diseases). We developed a fluorescent probing system for tracing protein aggregation process. ${ }^{156}$ Insulin is a biopolymer that readily undergoes fibrosis under the acidic conditions and was thus chosen as a model protein in our study. The aqueous mixture of native insulin and luminogen 73 is virtually non-fluorescent (Fig. 44A). In the presence of fibrillar insulin, however, $\mathbf{7 3}$ becomes emissive, for its interaction with the insulin aggregates activates its RIR process.

The distinct contrast in the emission efficiency permits clear discrimination between the native and denatured forms of insulin and enables tracking of its fibrosis process. The trajectory of the variation in the PL intensity implies that the fibrosis process proceeds through three steps, i.e., induction (I), exponential (II) and plateau stages (III; Fig. 44B). ${ }^{156}$ The amyloid fibrils stained by $\mathbf{7 3}$ can be readily observed under fluorescence microscope, thanks to the bright light emitted from the bound aggregates of 73. Due to the excellent watermiscibility of the unbound free molecules of $\mathbf{7 3}$, virtually no background emission is observed in the fluorescence image, as can be seen from the inset of Fig. 44B. ${ }^{156}$

\section{Summary and prospect}

AIE luminogens are a group of molecules whose emissions are induced by aggregate formation. In our previous Feature Article, we reviewed our own work in the area of research. ${ }^{29}$ In this critical review, we have summarised the progress made mainly by other research groups. We have presented typical examples of different categories of AIE luminogens, discussed their structure-property relationships, looked into their mechanistic aspects from experimental and theoretical viewpoints, derived new strategies and processes for solving ACQ problem and creating AIE luminogens, and illustrated their technological, especially optoelectronic and biological, applications.

It has been a general belief that $\pi$-conjugated molecules with planar conformations emit efficiently. This belief has formed the structural basis for conventional luminophores, many of which are disc-like polynuclear molecules. These luminophores, however, experience strong intermolecular interactions in the condensed phase. This has led to the early observation of the concentration quenching effect by Förster and Kasper, ${ }^{6,7,157}$ which has through time evolved into the consensus that aggregate formation is detrimental to light emission. The revelation of the beneficial effects of the AIE processes cleans the name of chromophore aggregation and proves that aggregation can work to our advantage through judicious structural design.

AIE luminogens are usually non-planar molecules that are non-emissive in the solution state. They are thus likely to be eliminated in the early stage of a screening process because, as discussed in the Introduction part, photophysical property evaluations have usually been done in dilute solutions. It has been a common law that a poor emitter in the solution state will not emit efficiently in the solid state. The AIE effect teaches us that this is not necessarily true. A poor emitter in a dilute solution may not be a "useless stone" but could be a "shining gold". This should help broaden our search avenues for efficient light-emitting materials in the solid state, which can be used to fabricate useful electro-optical devices such as OLEDs.

The AIE luminogens enjoy a great diversity in structure, ranging from pure hydrocarbons, organometallic complexes, small molecules, large polymers, systems with extended $\pi$-conjugations to those even without typical chromophores. Evidently, AIE is not an odd effect, as it seems to be, but a ubiquitous phenomenon. Though the earlier AIE luminogens were discovered by serendipity, the later AIE systems have been developed largely by design, mostly through judicious utilization of the RIR process. Many of the luminogens (e.g., TPE) can easily be functionalized further or structurally tailored by simple reactions and procedures to cater for the needs of various technological applications.

The study of the AIE phenomenon is of academic value. Whilst there are matured theories to explain the ACQ effect, new models need to be built to explicate the AIE process. In this review, we have correlated conformational planarity and structural rigidity of an AIE luminogen with its emission behaviour. It is noted that a majority of the experimental and computational data obtained so far support the RIR model. The mechanistic picture of the AIE process has thus become pretty clear now: the dynamic intramolecular motions, also known as the low-frequency modes of the reorganization energy, wipe out the excitons of the AIE luminogens, while the RIR process activated by the aggregate formation greatly boosts their light emissions. 


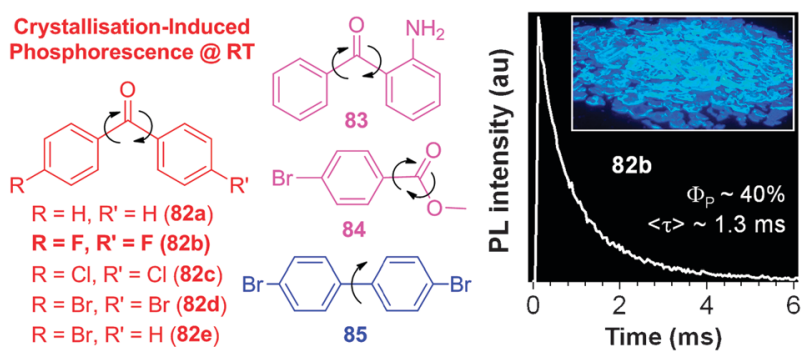

Fig. 45 Examples of room-temperature organic phosphorogens (82-85), whose emissions are induced by crystal formation. Phosphorescence decay curve of 4,4'-difluorobenzophenone (82b) and photograph of its crystals taken under UV illumination. Reproduced with permission from ref. 158. Copyright (2010) American Chemical Society.

Various methods have been used to tackle the ACQ issue, all aimed at preventing aggregate formation. Unfortunately, however, these conventional approaches have met with only limited success, as they are wrestling with a natural process. The ACQ effect is often lessened with the generation of new troubles. ${ }^{15-19}$ Our new strategies based on harnessing the aggregation process can solve the ACQ problem without causing any adverse effects. The integration of AIE units (e.g., TPE) with ACQ ones (e.g., carbazole) creates AIE luminogens that are free of ACQ effect, while preserving excellent functional properties of the components.

The AIE effect is practically useful. In the AIE systems, one can buoyantly take advantage of aggregate formation, instead of fighting against it. The AIE effect permits the use of highly concentrated solutions of the luminogens and their nanoaggregate suspensions in the aqueous media for sensing applications. In comparison to the turn-off probes based on the ACQ effect of the conventional luminophores in the dilute solutions, the sensors based on the solutions and suspensions of AIE luminogens are more sensitive. The turn-on nature of the AIE sensors makes them promising for field trial, on-site screening, household testing, etc. The AIE luminogens are emissive in the crystalline state, which is helpful to the fabrication of EL devices, as evidenced by the outstanding device performances of the OLEDs constructed from the AIE luminogens. ${ }^{28,29,75,125}$

Further investigation of the AIE effect may lead to more surprises, while elaborate exploration of the utilities of the RIR process may make the impossible possible. An example supporting this prospect is the discovery of efficient organic phosphorescence at room temperature, which has long been regarded as a "mission impossible", as triplet excited states are highly susceptible to the thermally activated molecular motions. Efficient phosphorescence, however, has been achieved recently in the crystals of $\mathbf{8 2}$ and $\mathbf{8 3}$ under ambient conditions (Fig. 45). The phosphorogens are firmly fixed in the crystal lattices, with the aid of multiple $\mathrm{C}-\mathrm{H} \cdots \mathrm{O}, \mathrm{N}-\mathrm{H} \cdots \mathrm{O}$, $\mathrm{C}-\mathrm{Br} \cdots \mathrm{Br}-\mathrm{C}, \mathrm{C}-\mathrm{H} \cdots \pi$ and $\mathrm{C}-\mathrm{H} \cdots \mathrm{X}(\mathrm{X}=$ halogen $)$ hydrogen bonds between the molecules. ${ }^{158}$ The molecular conformations are rigidified to such an extent that it enables the triplet excited states to undergo radiative transitions even at room temperature. Similarly efficient phosphorescence is observed in the crystals of phosphorogens $\mathbf{8 4}$ and $\mathbf{8 5}$ (Fig. 45) ${ }^{158}$ as well as halogenated aromatic aldehydes and their co-crystals. ${ }^{159}$
As a final remark, the AIE research is fast developing in recent years, resulting in the accumulation of a wealth of information on structural design of AIE luminogens and mechanistic understanding of AIE processes. ${ }^{29,75,125}$ During the preparation of this critical review, we have noted with great pleasure the publications of many excellent papers in various journals. However, since we cannot cover all the reported work in this article due to the space limitation, we are forced to be highly selective in choosing examples for discussions. As compensation to the loss, we here provide a list of representative papers recently published by different research teams in the reference section for interested readers to retrieve. ${ }^{160-183}$ Although much work has been done, many possibilities still remain to be explored. We enthusiastically look forward to the new advancements in this exciting area of research.

\section{Acknowledgements}

This work was partially supported by the Research Grants Council of Hong Kong (HKUST2/CRF/10 and 603509), the University Grants Committee of Hong Kong (AOE/P-03/08-1), the National Science Foundation of China (20974028), the Innovation and Technology Commission of Hong Kong (ITP/008/09NP and ITS/168/09) and the Ministry of Science and Technology of China (2009CB623605). B. Z. T. thanks the Cao Guangbiao Foundation of Zhejiang University for support.

\section{References}

1 S. W. Thomas III, G. D. Joly and T. M. Swager, Chem. Rev., 2007, 107, 1339.

2 F. J. M. Hoeben, P. Jonkheijm, E. W. Meijer and A. P. H. J. Schenning, Chem. Rev., 2005, 105, 1491.

3 U. H. F. Bunz, Chem. Rev., 2000, 100, 1605.

4 F. Hide, M. A. Diaz-Garcia, B. J. Schwartz and A. J. Heeger, Acc. Chem. Res., 1997, 30, 430.

5 S. M. Borisov and O. S. Wolfbeis, Chem. Rev., 2008, 108, 423.

6 Photonic Research Systems: http://www.prsbio.com/index.html (last accessed on 15 February 2011).

7 J. B. Birks, Photophysics of Aromatic Molecules, Wiley, London, 1970.

8 Z. Zhelev, H. Ohba and R. Bakalova, J. Am. Chem. Soc., 2006, 128, 6324.

9 R. Bakalova, Z. Zhelev, I. Aoki, H. Ohba, Y. Imai and I. Kanno, Anal. Chem., 2006, 78, 5925.

10 Fluorescence Sensors and Biosensors, ed. R. B. Thompson, CRC, Boca Raton, 2006.

11 C. W. Tang and S. A. Vanslyke, Appl. Phys. Lett., 1987, 51, 913.

12 Advanced Concepts in Fluorescence Sensing, ed. C. D. Geddes and J. R. Lakopwicz, Springer, Norwell, 2005.

13 E. A. Jares-Erijman and T. M. Jovin, Nat. Biotechnol., 2003, 21, 1387.

14 H. Saigusa and E. C. Lim, J. Phys. Chem., 1995, 99, 15738.

15 J. Wang, Y. Zhao, Y. C. Dou, H. Sun, P. Xu, K. Ye, J. Zhang, S. Jiang, F. Li and Y. Wang, J. Phys. Chem. B, 2007, 111, 5082.

16 S. Hecht and J. M. J. Frechet, Angew. Chem., Int. Ed., 2001, 40, 74 .

17 B. T. Nguyen, J. E. Gautrot, C. Ji, P.-L. Brunner, M. T. Nguyen and X. X. Zhu, Langmuir, 2006, 22, 4799.

18 L. Chen, S. Xu, D. McBranch and D. Whitten, J. Am. Chem. Soc., 2000, 122, 9302.

19 P. N. Taylor, M. J. O'Connell, L. A. McNeill, M. J. Hall, R. T. Aplin and H. L. Anderson, Angew. Chem., Int. Ed., 2000, 39, 3456 . 
20 J. Luo, Z. Xie, J. W. Y. Lam, L. Cheng, H. Chen, C. Qiu, H. S. Kwok, X. Zhan, Y. Liu, D. Zhu and B. Z. Tang, Chem. Commun., 2001, 1740.

21 B. Z. Tang, X. Zhan, G. Yu, P. P. S. Lee, Y. Liu and D. Zhu, J. Mater. Chem., 2001, 11, 2974.

22 J. W. Chen, B. Xu, X. Y. Ouyang, B. Z. Tang and Y. Cao, J. Phys. Chem. A, 2004, 108, 7522.

23 H. Tong, Y. Hong, Y. Dong, Y. Ren, M. Haeussler, J. W. Y. Lam, K. S. Wong and B. Z. Tang, J. Phys. Chem. B, 2007, 111, 2000.

24 H. Tong, Y. Dong, Y. Hong, M. Haeussler, J. W. Y. Lam, H. H. Y. Sung, X. Yu, J. Sun, I. D. Williams, H. S. Kwok and B. Z. Tang, J. Phys. Chem. C, 2007, 111, 2287.

25 Q. Zeng, Z. Li, Y. Dong, C. Di, A. Qin, Y. Hong, L. Ji, Z. Zhu, C. K. W. Jim, G. Yu, Q. Li, Z. Li, Y. Liu, J. Qin and B. Z. Tang, Chem. Commun., 2007, 70.

26 Y. Dong, J. W. Y. Lam, A. Qin, J. Liu, Z. Li, B. Z. Tang, J. Sun and H. S. Kwok, Appl. Phys. Lett., 2007, 91, 01111.

27 M. Freemantle, Chem. Eng. News, 2001, 79, 29.

28 M. Wang, G. Zhang, D. Zhang, D. Zhu and B. Z. Tang, J. Mater. Chem., 20, 1858.

29 Y. Hong, J. W. Y. Lam and B. Z. Tang, Chem. Commun., 2009, 4332.

30 Y. T. Wu, M. Y. Kuo, Y. T. Chang, C. C. Shin, T. C. Wu, C. C. Tai, T. H. Cheng and W. S. Liu, Angew. Chem., Int. Ed., 2008, 47, 9891.

31 J. Chen, C. C. W. Law, J. W. Y. Lam, Y. Dong, S. M. F. Lo, I. D. Williams, D. Zhu and B. Z. Tang, Chem. Mater., 2003, 15,1535 .

32 S. Kim, Q. Zheng, G. He, D. J. Bharali, H. E. Pudavar, A. Baev and P. N. Prasad, Adv. Funct. Mater., 2006, 16, 2317.

33 C. J. Bhongale, C. W. Chang, C. S. Lee, E. W. G. Diau and C. S. Hsu, J. Phys. Chem. B, 2005, 109, 13472.

34 S. Grimme, Angew. Chem., Int. Ed., 2008, 47, 3430.

35 H. Saigusa and E. C. Lim, J. Phys. Chem., 1995, 99, 15738.

36 K. Itami, Y. Ohashi and J. I. Yoshida, J. Org. Chem., 2005, 70, 2778 .

37 Z. Xie, B. Yang, W. Xie, L. Liu, F. Shen, H. Wang, X. Yang, Z. Wang, Y. Li, M. Hanif, G. Yang, L. Ye and Y. Ma, J. Phys. Chem. B, 2006, 110, 20993.

38 Z. Wang, H. Shao, J. Ye, L. Tang and P. Lu, J. Phys. Chem. B, 2005, 109, 19627.

39 H. Jiating, X. Bin, C. Feipeng, X. Haijian, L. Kunpeng, Y. Ling and T. Wenjing, J. Phys. Chem. C, 2009, 113, 9892.

40 M. Shimizu, Y. Takeda, M. Higashi and T. Hiyama, Angew. Chem., Int. Ed., 2009, 48, 3653.

41 C. X. Yuan, X. T. Tao, L. Wang, J. X. Yang and M. H. Jiang, J. Phys. Chem. C, 2009, 113, 6809.

42 Z. Yang, Z. Chi, T. Yu, X. Zhang, M. Chen, B. Xu, S. Liu, Y. Zhang and J. Xu, J. Mater. Chem., 2009, 19, 5541.

43 K. Hirano, S. Minakata, M. Komatsu and J. Mizuguchi, J. Phys. Chem. A, 2002, 106, 4868.

44 Z. J. Ning, Z. Chen, Q. Zhang, Y. L. Yan, S. X. Qian, Y. Cao and H. Tian, Adv. Funct. Mater., 2007, 17, 3799.

45 Y. Liu, X. T. Tao, F. Z. Wang, X. N. Dang, D. C. Zou, Y. Ren and M. H. Jiang, J. Phys. Chem. C, 2008, 112, 3975.

46 T. Baumgartner and R. Reau, Chem. Rev., 2006, 106, 4681.

47 K. Shiraishi, T. Kashiwabara, T. Sanji and M. Tanaka, New J. Chem., 2009, 33, 1680.

48 H. C. Su, O. Fadhel, C. J. Yang, T. Y. Cho, C. Fave, M. Hissler, C. C. Wu and R. Reau, J. Am. Chem. Soc., 2006, 128, 983.

49 G. Qian, B. Dai, M. Luo, D. Yu, J. Zhan, Z. Zhang, M. Dongge and Z. Y. Wang, Chem. Mater., 2008, 20, 6208.

50 B. K. An, S. K. Kwon, S. D. Jung and S. Y. Park, J. Am. Chem. Soc., 2002, 124, 14410.

51 J. Xu, L. Wen, W. Zhou, J. Lv, Y. Guo, M. Zhu, H. Liu, Y. Li and L. Jiang, J. Phys. Chem. C, 2009, 113, 5924.

52 S. Jayanty and T. P. Radhakrishnan, Chem.-Eur. J., 2004, 10, 791 .

53 Y. Li, F. Li, H. Zhang, Z. Xie, W. Xie, H. Xu, B. Li, F. Shen, L. Ye, M. Hanif, D. Ma and Y. Ma, Chem. Commun., 2007, 231.

54 H. C. Yeh, W. C. Wu, Y. S. Wen, D. C. Dai, J. K. Wang and C. T. Chen, J. Org. Chem., 2004, 69, 6455.

55 S. S. Palayangoda, X. Cai, R. M. Adhikari and D. C. Neckers, Org. Lett., 2008, 10, 281.
56 M. Han and M. Hara, J. Am. Chem. Soc., 2005, 127, 10951.

57 T. Zhou, F. Li, Y. Fan, W. Song, X. Mu, H. Zhang and Y. Wang, Chem. Commun., 2009, 3199.

58 J. Xu, X. Liu, J. Lv, M. Zhu, C. Huang, W. Zhou, X. Yin, H. Liu, Y. Li and J. Ye, Langmuir, 2008, 24, 4231.

59 S. Qu, L. Zhao, Z. Yu, Z. Xiu, C. Zhao, P. Zhang, B. Long and M. Li, Langmuir, 2009, 25, 1713.

60 W. X. Tang, Y. Xiang and A. J. Tong, J. Org. Chem., 2009, 74, 2163.

61 S. Scheiner and V. M. Kolb, Proc. Natl. Acad. Sci. U. S. A., 1980, 77, 5602.

62 Y. Qian, S. Y. Li, G. Q. Zhang, Q. Wang, S. Q. Wang, H. J. Xu, C. Z. Li, Y. Li and G. Q. Yang, J. Phys. Chem. B, 2007, 111, 5861.

63 T. Mutai, H. Tomoda, T. Ohkawa, Y. Yabe and K. Araki, Angew. Chem., Int. Ed., 2008, 47, 9522.

$64 \mathrm{H}$. Birkedal and P. Pattison, Acta Crystallogr., Sect. C: Cryst. Struct. Commun., 2006, c62, o139.

65 P. Chen, R. Lu, P. Xue, T. Xu, G. Chen and Y. Zhao, Langmuir, 2009, 25, 8395.

66 S. Y. Ryu, S. Kim, J. Seo, Y. W. Kim, O. H. Kwon, D. J. Jang and S. Y. Park, Chem. Commun., 2004, 70.

67 Y. Chen, Y. Lv, Y. Han, B. Zhu, F. Zhang, Z. Bo and C. Y. Liu, Langmuir, 2009, 25, 8548.

68 C. Bao, R. Lu, M. Jin, P. Xue, C. Tan, G. Liu and Y. Zhao, Org. Biomol. Chem., 2005, 3, 2508.

69 J. Liu, J. W. Y. Lam and B. Z. Tang, Chem. Rev., 2009, 109, 5799.

70 A. Qin, C. K. W. Jim, Y. Tang, J. W. Y. Lam, J. Liu, F. Mahtab, P. Gao and B. Z. Tang, J. Phys. Chem. B, 2008, 112, 9281.

71 W. Z. Yuan, H. Zhao, X. Y. Shen, F. Mahtab, J. W. Y. Lam, J. Z. Sun and B. Z. Tang, Macromolecules, 2009, 42, 9400.

72 A. Qin, J. W. Y. Lam and B. Z. Tang, Chem. Soc. Rev., 2010, 39, 2522.

73 S. Kim, H. E. Pudavar, A. Bonoiu and P. N. Prasad, Adv. Mater., 2007, 19, 3791.

74 K. Kokado and Y. Chujo, Macromolecules, 2009, 42, 1418.

75 J. Liu, J. W. Y. Lam and B. Z. Tang, J. Inorg. Organomet. Polym. Mater., 2009, 19, 249.

76 P. Lu, J. W. Y. Lam, J. Liu, C. K. W. Jim, W. Yuan, N. Xie, Y. Zhong, Q. Hu, K. S. Wong, K. K. L. Cheuk and B. Z. Tang, Macromol. Rapid Commun., 2010, 31, 834.

77 J. Liu, Y. Zhong, J. W. Y. Lam, P. Lu, Y. Hong, Y. Yu, Y. Yue, F. Mahtab, H. H. Y. Sung, I. D. Williams, K. S. Wong and B. Z. Tang, Macromolecules, 2010, 43, 4921.

78 Y. Ruff, E. Buhler, S. J. Candau, E. Kesselman, Y. Talmon and J. M. Lehn, J. Am. Chem. Soc., 2010, 132, 2573.

79 Y. Ruff and J. M. Lehn, Angew. Chem., Int. Ed., 2008, 47, 3556.

80 C. M. Xing, J. W. Y. Lam, A. Qin, Y. Dong, M. Haussler, W. T. Yang and B. Z. Tang, Polym. Mater. Sci. Eng., 2007, 96, 418.

81 Y. Hong, C. Xing, Y. Yu, J. W. Y. Lam and B. Z. Tang, Abstracts of the 17th Symposium on Chemistry Postgraduate Research in Hong Kong, Hong Kong, 2010, O-78.

82 A. Pucci, R. Rausa and F. Ciardelli, Macromol. Chem. Phys., 2008, 209, 900

83 A. Y. Y. Tam, K. M. C. Wong and V. W. W. Yam, J. Am. Chem. Soc., 2009, 131, 6253.

84 M. X. Zhu, W. Lu, N. Y. Zhu and C. M. Che, Chem.-Eur. J., 2008, 14, 9736.

85 B. Manimaran, P. Thanasekaran, T. Rajendran, R. J. Lin, I. J. Chang, G. H. Lee, S. M. Peng, S. Rajagopal and K. L. Lu, Inorg. Chem., 2002, 41, 5323.

86 J. S. Y. Lau, P. K. Lee, K. H. K. Tsang, C. H. C. Ng, Y. W. Lam, S. H. Cheng and K. K. W. Lo, Inorg. Chem., 2009, 48, 708.

87 Q. Zhao, L. Li, F. Li, M. Yu, Z. Liu, T. Yi and C. Huang, Chem. Commun., 2008, 685.

88 Y. You, H. S. Huh, K. S. Kim, S. W. Lee, D. Kim and S. Y. Park, Chem. Commun., 2008, 3998.

89 K. Huang, H. Wu, M. Shi, F. Li, T. Yi and C. Huang, Chem. Commun., 2009, 1243.

90 R. Kabe, H. Nakanotani, T. Sakanoue, M. Yahiro and C. Adachi, Adv. Mater., 2009, 21, 4034.

91 M. Shimizu, H. Tatsumi, K. Mochida, K. Shimono and T. Hiyama, Chem--Asian J., 2009, 4, 1289.

92 G. Yui, S. Yin, Y. Liu, J. Chen, X. Xu, X. Sun, D. Ma, X. Zhan, Q. Peng, Z. Shuai, B. Tang, D. Zhu, W. Fang and Y. Luo, J. Am. Chem. Soc., 2005, 127, 6335. 
93 S. Yin, Q. Peng, Z. Shuai, W. Fang, Y. H. Wang and Y. Luo, Phys. Rev. B: Condens. Matter Mater. Phys., 2006, 73, 205409.

94 Q. Peng, Y. Yi, Z. Shuai and J. Shao, J. Am. Chem. Soc., 2007, 129, 9333.

95 Y. Yamaguchi, Y. Matsubara, T. Ochi, T. Wakamiya and Z. I. Yoshida, J. Am. Chem. Soc., 2008, 130, 13867.

96 A. Loudet and K. Burgess, Chem. Rev., 2007, 107, 4891.

97 R. Hu, E. Lager, A. Aguilar-Aguilar, J. Liu, J. W. Y. Lam, H. H. Y. Sung, I. D. Williams, Y. Zhong, K. S. Wong, E. Peña-Cabrera and B. Z. Tang, J. Phys. Chem. C, 2009, 113, 15845.

98 M. Katkevics, S. Yamaguchi, A. Toshimitsu and K. Tamao, Organometallics, 1998, 17, 5796.

99 D. A. Shultz and M. A. Fox, J. Am. Chem. Soc., 1989, 111, 6311. 100 G. Oster and Y. Nishijima, J. Am. Chem. Soc., 1956, 78, 1581.

101 M. Ashsap El-Bayomnf and F. M. Abdel Haiim, J. Chem. Phys., 1968, 48, 2536.

102 N. I. Nijegorodov and W. S. Downey, J. Phys. Chem., 1994, 98, 5639.

103 J. Dong, K. M. Solntsev and L. M. Tolbert, J. Am. Chem. Soc., 2009, 131, 662.

104 L. Wu and K. Burgess, J. Am. Chem. Soc., 2008, 130, 4089.

105 P. Naumov, J. Kowalik, K. M. Solntsev, A. Baldridge, J. S. Moon, C. Kranz and L. M. Tolbert, J. Am. Chem. Soc., 2010, 132, 5845

106 A. Iida and S. Yamaguchi, Chem. Commun., 2009, 3002.

107 C. David Sherrill, T. Takatani and E. G. Hohenstein, J. Phys. Chem. A, 2009, 113, 10146.

108 E. Gagnon, T. Maris, P. M. Arseneault, K. E. Maly and J. D. Wuest, Cryst. Growth Des., 10, 648.

109 E. Q. Procopio, M. Mauro, M. Panigati, D. Donghi, P. Mercandelli, A. Sironi, G. D'Alfonso and L. De Cola, J. Am. Chem. Soc., 2010, 132, 14397.

110 E. W. Debler, G. F. Kaufmann, M. M. Meijler, A. Heine, J. M. Mee, G. Pljevaljcic, A. J. Di Bilio, P. G. Schultz, D. P. Millar, K. D. Janda, I. A. Wilson, H. B. Gray and R. A. Lerner, Science, 2008, 319, 1232.

111 B. A. Armitage and P. B. Berget, Science, 2008, 319, 1195.

112 W. Z. Yuan, P. Lu, S. Chen, J. W. Y. Lam, Z. Wang, Y. Liu, H. S. Kwok and B. Z. Tang, Adv. Mater., 2010, 22, 2159.

113 Z. Zhao, S. Chen, X. Shen, F. Mahtab, Y. Yu, P. Lu, J. W. Y. Lam, H. S. Kwok and B. Z. Tang, Chem. Commun., 2010, 46, 686 .

114 R. Katoh, S. Katoh, A. Furube, K. Tokumaru and M. Kotani, J. Phys. Chem. C, 2009, 113, 2961.

115 Z. Zhao, S. Chen, J. W. Y. Lam, P. Lu, Y. Zhong, K. S. Wong, H. S. Kwok and B. Z. Tang, Chem. Commun., 2010, 46, 2221.

116 T. M. Figueira-Duarte, P. G. Del Rosso, R. Trattnig, S. Sax, E. J. W. List and K. Mullen, Adv. Mater., 22, 990.

117 Z. Zhao, P. Lu, J. W. Y. Lam, Z. Wang, C. Y. K. Chan, H. H. Y. Sung, I. D. Williams, Y. Ma and B. Z. Tang, Chem. Sci., 2011, 2, 672.

118 J. N. Moorthy, P. Natarajan, P. Venkatakrishnan, D. F. Huang and T. J. Chow, Org. Lett., 2007, 9, 5215.

119 H. Y. Chen, W. Y. Lam, J. D. Luo, Y. L. Ho, B. Z. Tang, D. B. Zhu, M. Wong and H. S. Kwok, Appl. Phys. Lett., 2002, 81, 574.

120 J. C. Sanchez and W. C. Trogler, J. Mater. Chem., 2008, 18, 3143.

121 E. L. Que, D. W. Domaille and C. J. Chang, Chem. Rev., 2008, 108, 1517.

122 M. Sameiro and T. Gonçalves, Chem. Rev., 2009, 109, 190.

123 V. S. Vyas and R. Rathore, Chem. Commun., 2010, 46, 1065.

124 Z. Ning and H. Tian, Chem. Commun., 2009, 5483.

125 Z. Zhao, J. W. Y. Lam and B. Z. Tang, Curr. Org. Chem., 2010, 14, 2109.

126 H. C. Yeh, S. J. Yeh and C. T. Chen, Chem. Commun., 2003, 2632.

127 S. J. Toal, K. A. Jones, D. Magde and W. C. Trogler, J. Am. Chem. Soc., 2005, 127, 11661.

128 Y. Hong, M. Haeussler, J. W. Y. Lam, Z. Li, K. K. Sin, Y. Dong, H. Tong, J. Liu, A. Qin, R. Renneberg and B. Z. Tang, Chem.-Eur. J., 2008, 14, 6428.

129 Y. Hong, H. Xiong, J. W. Y. Lam, M. Haessler, J. Liu, Y. Yu, Y. Zhong, H. H. Y. Sung, I. D. Williams, K. S. Wong and B. Z. Tang, Chem.-Eur. J., 2010, 16, 1232.

130 Y. Liu, Y. Tang, N. N. Barashkov, I. S. Irgibaeva, J. W. Y. Lam, R. Hu, D. Birimzhanova, Y. Yu and B. Z. Tang, J. Am. Chem. Soc., 2010, 132, 13951.
131 T. L. Andrew and T. M. Swager, J. Am. Chem. Soc., 2007, 129, 7254.

132 S. J. Toal, D. Magde and W. C. Trogler, Chem. Commun., 2005, 5465.

133 K. Shiraishi, T. Sanji and M. Tanaka, ACS Appl. Mater. Interfaces, 2009, 1, 1379.

134 J. Liu, Y. Zhong, P. Lu, Y. Hong, J. W. Y. Lam, M. Faisal, Y. Yu, K. S. Wong and B. Z. Tang, Polym. Chem., 2010, 1, 426.

135 Y. S. Zheng and Y. J. Hu, J. Org. Chem., 2009, 74, 5660.

136 Y. Liu, Y. Yu, J. W. Y. Lam, Y. Hong, M. Faisal, W. Z. Yuan and B. Z. Tang, Chem.-Eur. J., 2010, 16, 8433.

137 H. Lis and N. Sharon, Chem. Rev., 1998, 98, 637.

138 T. Sanji, K. Shiraishi and M. Tanaka, ACS Appl. Mater. Interfaces, 2009, 1, 270.

139 M. C. Zhao, M. Wang, H. Liu, D. S. Liu, G. X. Zhang, D. Q. Zhang and D. B. Zhu, Langmuir, 2009, 25, 676.

140 H. Tong, Y. N. Hong, Y. Q. Dong, M. Haeussler, Z. Li, J. W. Y. Lam, Y. P. Dong, H. H. Y. Sung, I. D. Williams and B. Z. Tang, J. Phys. Chem. B, 2007, 111, 11817.

141 Y. Hong, C. Feng, Y. Yu, J. Liu, J. W. Y. Lam, K. Q. Luo and B. Z. Tang, Anal. Chem., 2010, 82, 7035.

142 W. C. Wu, C. Y. Chen, Y. Tian, S. H. Jang, Y. Hong, Y. Liu, R. Hu, B. Z. Tang, Y. T. Lee, C. T. Chen, W. C. Chen and A. K. Y. Jen, Adv. Funct. Mater., 2010, 20, 1413.

143 M. Faisal, Y. Hong, J. Liu, Y. Yu, J. W. Y. Lam, A. Qin, P. Lu and B. Z. Tang, Chem.-Eur. J., 2010, 16, 4266.

144 F. Mahtab, Y. Yu, J. W. Y. Lam, J. Liu, B. Zhang, P. Lu, X. Zhang and B. Z. Tang, Adv. Funct. Mater., 2011, 21, DOI: 10.1002/adfm.201002572.

145 K. H. Cheng, Y. Zhong, B. Y. Xie, Y. Q. Dong, Y. Hong, J. Z. Sun, B. Z. Tang and K. S. Wong, J. Phys. Chem. C, 2008, 112, 17507.

146 B. K. An, S. H. Gihm, J. W. Chung, C. R. Park, S. K. Kwon and S. Y. Park, J. Am. Chem. Soc., 2009, 131, 3950.

147 Z. Zhao, Z. Wang, P. Lu, C. Y. K. Chan, D. Liu, J. W. Y. Lam, H. H. Y. Sung, I. D. Williams, Y. Ma and B. Z. Tang, Angew. Chem., Int. Ed., 2009, 48, 7608.

148 J. W. Y. Lam and B. Z. Tang, Acc. Chem. Res., 2005, 38, 745.

149 J. W. Y. Lam and B. Z. Tang, J. Polym. Sci., Part A: Polym. Chem., 2003, 41, 2607.

150 C. Weder, C. Sarwa, A. Montali, C. Bastiaansen and P. Smith, Science, 1998, 279, 835.

151 S. Laschat, A. Baro, N. Steinke, F. Giesselmann, C. Hagele, G. Scalia, R. Judele, E. Kapatsina, S. Sauer, A. Schreivogel and M. Tosoni, Angew. Chem., Int. Ed., 2007, 46, 4832.

152 H. Ito, T. Saito, N. Oshima, N. Kitamura, S. Ishizaka, Y. Hinatsu, M. Wakeshima, M. Kato, K. Tsuge and M. Sawamura, J. Am. Chem. Soc., 2008, 130, 10044.

153 D. Avobenzone, G. Zhang, J. Lu, M. Sabat and C. L. Fraser, J. Am. Chem. Soc., 2010, 132, 2160.

154 J. W. Chung, B. K. An and S. Y. Park, Chem. Mater., 2008, 20, 6750 .

155 L. Tang, J. K. Jin, A. Qin, W. Zhang Yuan, Y. Mao, J. Mei, J. Zhi Sun and B. Z. Tang, Chem. Commun., 2009, 4974.

156 B. Z. Tang, Y. Hong and S. Chen, US Provisional Patent Appln No. 61/457,207, 2011.

157 Th. Förster and K. Kasper, Z. Phys. Chem. (Munich), 1954, 1, 275.

158 Y. Z. Yuan, X. Y. Shen, H. Zhao, J. W. Y. Lam, L. Tang, P. Lu, C. Wang, Y. Liu, Z. Wang, Q. Zheng, J. Z. Sun, Y. Ma and B. Z. Tang, J. Phys. Chem. C, 2010, 114, 6090.

159 O. Bolton, K. Lee, H. J. Kim, K. Y. Lin and J. Kim, Nat. Chem., $2011,3,207$.

160 T. Hirose, K. Higashiguchi and K. Matsuda, Chem.-Asian J., 2011, 6, 1057.

161 X. B. Du and Z. Y. Wang, Chem. Commun., 2011, 47, 4276.

162 Y. Jin, Y. B. Xu, Y. L. Liu, L. Y. Wang, H. F. Jiang, X. J. Li and D. R. Cao, Dyes Pigm., 2011, 90, 311.

163 H. Y. Li, X. Q. Zhang, Z. G. Chi, B. J. Xu, W. Zhou, S. W. Liu, Y. Zhang and J. R. Xu, Org. Lett., 2011, 13, 556.

164 M. Shimizu and T. Hiyama, Chem.-Asian J., 2010, 5, 1516.

165 V. S. Vyas and R. Rathore, Chem. Commun., 2010, 46, 1065.

166 Y. Qian, M. M. Cai, L. H. Xie, G. Q. Yang, S. K. Wu and W. Huang, ChemPhysChem, 2011, 12, 397.

167 J. H. Wan, L. Y. Mao, Y. B. Li, Z. F. Li, H. Y. Qiu, C. Wang and G. Q. Lai, Soft Matter, 2010, 6, 3195.

168 B. Wang, Y. C. Wang, J. L. Hua, Y. H. Jiang, J. H. Huang, S. X. Qian and H. Tian, Chem.-Eur. J., 2011, 17, 2647. 
169 P. An, Z. F. Shi, W. Dou, X. P. Cao and H. L. Zhang, Org. Lett., 2010, 12, 4364.

170 H. H. Fang, Q. D. Chen, J. Yang, H. Xia, B. R. Gao, J. Feng, Y. G. Ma and H. B. Sun, J. Phys. Chem. C, 2010, 114, 11958.

171 R. Hu, S. Y. Li, Y. Zeng, J. P. Chen, S. Q. Wang, Y. Li and G. Q. Yang, Phys. Chem. Chem. Phys., 2011, 13, 2044.

172 T. He, X. T. Tao, J. X. Yang, D. Guo, H. B. Xia, J. O. Jia and M. H. Jiang, Chem. Commun., 2011, 47, 2907.

173 K. A. N. Upamali, L. A. Estrada, P. K. De, X. C. Cai, J. A. Krause and D. C. Neekers, Langmuir, 2011, 27, 1573.

174 L. Q. Li, P. Gao, K. C. Schuermann, S. Ostendorp, W. C. Wang, C. A. Du, Y. Lei, H. Fuchs, L. De Cola, K. Mullen and L. F. Chi, J. Am. Chem. Soc., 2010, 132, 8807.

175 K. Miyamoto, T. Sawada, H. Jintoku, M. Takafuji, T. Sagawa and H. Ihara, Tetrahedron Lett., 2010, 51, 4666.

176 J. Chahine, N. Saffon, M. Cantuel and S. Fery-Forgues, Langmuir, 2011, 27, 2844.
177 J. W. Levell, A. Ruseckas, J. B. Henry, Y. Wang, A. D. Stretton, A. R. Mount, T. H. Galow and I. D. W. Samuel, J. Phys. Chem. A, 2010, 114, 13291.

178 S. Kamino, Y. Horio, S. Komeda, K. Minoura, H. Ichikawa, J. Horigome, A. Tatsumi, S. Kaji, T. Yamaguchi, Y. Usami, S. Hirota, S. Enomoto and Y. Fujita, Chem. Commun., 2010, 46, 9013.

179 Y. Chen, W. C. Xu, J. F. Kou, B. L. Yu, X. H. Wei, H. Chao and L. N. Ji, Inorg. Chem. Commun., 2010, 13, 1140.

180 Y. Liu, C. Deng, L. Tang, A. Qin, R. Hu, J. Z. Sun and B. Z. Tang, J. Am. Chem. Soc., 2011, 133, 660.

181 H. H. Lin, Y. C. Chan, J. W. Chen and C. C. Chang, J. Mater. Chem., 2011, 21, 3170.

182 R. H. Chien, C. T. Lai and J. L. Hong, J. Phys. Chem. C, 2011, $115,5958$.

183 J. X. Wang, Q. Chen, N. Bian, F. Yang, J. Sun, A. D. Qi, C. G. Yan and B. H. Han, Org. Biomol. Chem., 2011, 9, 2219. 